\title{
THE OPTICAL DEPTH OF H II REGIONS IN THE MAGELLANIC CLOUDS
}

\author{
E. W. Pellegrini ${ }^{1}$, M. S. Oey ${ }^{1}$, P. F. Winkler ${ }^{2}$, S. D. Points ${ }^{3}$, R. C. Smith $^{3}$, A. E. Jaskot ${ }^{1}$, And J. Zastrow ${ }^{1}$ \\ ${ }^{1}$ Department of Astronomy, University of Michigan, 500 Church Street, Ann Arbor, MI 48109, USA; pelleger@umich.edu \\ ${ }^{2}$ Department of Physics, Middlebury College, Middlebury, VT 05753, USA \\ ${ }^{3}$ Cerro Tololo Inter-American Observatory, National Optical Astronomy Observatory, Casilla 603, La Serena, Chile \\ Received 2011 October 21; accepted 2012 March 1; published 2012 July 24
}

\begin{abstract}
We exploit ionization-parameter mapping (IPM) as a powerful tool to measure the optical depth of star-forming $\mathrm{H}$ II regions. Our simulations using the photoionization code CLOUDY and our new, SURFBRIGHT surface-brightness simulator demonstrate that this technique can directly diagnose most density-bounded, optically thin nebulae using spatially resolved emission-line data. We apply this method to the Large and Small Magellanic Clouds (LMC and SMC), using the data from the Magellanic Clouds Emission Line Survey. We generate new H II region catalogs based on photoionization criteria set by the observed ionization structure in the $[\mathrm{S} \mathrm{II}] /[\mathrm{O} \mathrm{III}]$ ratio and $\mathrm{H} \alpha$ surface brightness. The luminosity functions from these catalogs generally agree with those from H $\alpha$-only surveys. We then use IPM to crudely classify all the nebulae into optically thick versus optically thin categories, yielding fundamental new insights into Lyman-continuum $(\mathrm{LyC})$ radiation transfer. We find that in both galaxies, the frequency of optically thin objects correlates with $\mathrm{H} \alpha$ luminosity, and that the numbers of these objects dominate above $\log L /\left(\mathrm{erg} \mathrm{s}^{-1}\right) \geqslant 37.0$. The frequencies of optically thin objects are $40 \%$ and $33 \%$ in the LMC and SMC, respectively. Similarly, the frequency of optically thick regions correlates with $\mathrm{H}$ I column density, with optically thin objects dominating at the lowest $N(\mathrm{HI})$. The integrated escape luminosity of ionizing radiation is dominated by the largest regions and corresponds to luminosity-weighted, ionizing escape fractions from the $\mathrm{H}$ II region population of $\geqslant 0.42$ and $\geqslant 0.40$ in the LMC and SMC, respectively. These values correspond to global galactic escape fractions of $4 \%$ and $11 \%$, respectively. This is sufficient to power the ionization rate of the observed diffuse ionized gas in both galaxies. Since our optical depth estimates tend to be underestimates, and also omit the contribution from field stars without nebulae, our results suggest the possibility of significant galactic escape fractions of LyC radiation.
\end{abstract}

Key words: catalogs - H II regions - ISM: structure - Magellanic Clouds - radiative transfer - stars: massive

Online-only material: color figures, figure sets

\section{INTRODUCTION}

Few of the rich and complex disciplines in astrophysics affect our understanding of the universe as deeply as the diffusion of ionizing radiation from stars and its interaction with surrounding matter. Of all the known sinks and sources of energy, the ionizing radiation released by $\mathrm{O}$ stars during their short lives has great consequences by (1) determining the structure and energy balance of the interstellar medium (ISM) in galaxies, (2) generating diagnostics of stellar populations and interstellar conditions, and (3) providing an important source of the Lyman continuum $(\mathrm{LyC})$ radiation field during cosmic reionization.

The luminosity and spectral energy distribution (SED) of massive stars make them a powerful source of ionizing radiation within star-forming galaxies (e.g., Abbott 1982; Reynolds 1984). Their power has been demonstrated by studies of nearby galaxies which show that the $\mathrm{LyC}$ radiation from $\mathrm{O}$ stars embedded within $\mathrm{H}$ II regions, combined with those in the field, is luminous enough to balance the incessant recombination and cooling of the diffuse, warm ionized medium (WIM) in galaxies (e.g., Oey \& Kennicutt 1997; Hoopes \& Walterbos 2000; Oey et al. 2004; for a recent review of the WIM see Haffner et al. 2009). Radiative transfer calculations also demonstrate that injecting ionizing radiation from stars into the WIM not only heats the gas but also acts to decrease its cooling efficiency (Cantalupo 2010), preventing the catastrophic cooling of warm diffuse gas, which would lead to unregulated star formation (e.g., Parravano 1988; Ostriker et al. 2010). In these ways, ionizing stellar radiation can strongly influence both the ISM structure and star formation rates of galaxies.
The H Balmer recombination lines form beacons of star formation across the universe (e.g., Cowie \& Hu 1998). When ionizing photons are all absorbed by gas, $\mathrm{H}$ recombination lines are an accurate diagnostic of $Q\left(\mathrm{H}^{0}\right)$, the rate at which $\mathrm{H}$ ionizing radiation is produced by stars. Multiwavelength emission-line observations and theoretical stellar SEDs are routinely used with observed recombination rates to infer the stellar populations of distant galaxies (e.g., Sullivan et al. 2004; Iglesias-Páramo et al. 2004).

Ionizing radiation from stars may ultimately escape into the intergalactic medium (IGM) before being absorbed. This radiation may be an important source of the cosmic background UV field during the epoch of reionization, some time between redshift $z \sim 11$ (Komatsu et al. 2011) and $z \sim 6$ (Fan et al. 2002). During this time, star-forming galaxies are believed to contribute $10 \%-20 \%$ of their total ionizing radiation budget to sustain reionization because the UV and X-ray field from an active galactic nucleus alone was likely insufficient (Sokasian et al. 2003). Recent detections of faint $\mathrm{Ly} \alpha$ emitting galaxies by Dressler et al. (2011) support this view with evidence that aggregate $\mathrm{LyC}$ radiation from faint galaxies during this epoch is sufficient to sustain cosmic reionization.

Understanding the radiative transfer of LyC photons from massive stars is therefore a fundamental problem, and although they are well understood in general terms, Hil regions still present a computational challenge because of their greatly varying densities, small-scale structure, and irregular nature. Consequently, Paardekooper et al. (2011) identified radiative transfer of individual nebulae as the main bottleneck that limits our ability to determine the escape fraction of ionizing radiation 
from star-forming galaxies in cosmological simulations. It is thus imperative to understand radiation transport within $\mathrm{HII}$ regions if we are to understand fundamental properties of the universe.

There has been a variety of approaches to evaluate the optical depth of $\mathrm{H}$ II regions. The most direct method compares the ionization rate derived from $\mathrm{H} \alpha$ luminosities $L$ to that predicted from the observed ionizing stellar population. Using this approach, Oey \& Kennicutt (1997) found that up to half of all ionizing photons generated by stars escape H II regions to ionize the WIM, also known as diffuse ionized gas (DIG). However, theoretical predictions for the LyC photon emission rate $Q\left(\mathrm{H}^{0}\right)$ have decreased significantly (e.g., Martins et al. 2005; Smith et al. 2002) and are now generally consistent with the observed HiI region luminosities (e.g., Voges et al. 2008; Zastrow et al. 2011a). Clearly, until the ionizing fluxes and SEDs of massive stars are definitively established, comparing predicted and observed $L$ will be subject to large systematic uncertainties. Identifying all the ionizing stars is also difficult in regions with significant extinction and crowding.

Other studies attempt to evaluate nebular optical depth by modeling nebular emission lines from ions with different ionization potentials averaged over the entire $\mathrm{H}$ II region (e.g., Relaño et al. 2002; Iglesias-Páramo \& Muñoz-Tuñón 2002; Giammanco et al. 2004; Kehrig et al. 2011). However, inhomogeneous, optically thin nebulae may contain many optically thick cloudlets. Since the emission-line volume emissivity is proportional to the square of the electron density, the resulting spatially integrated spectra can be dominated by these dense clumps and resemble the spectrum of an optically thick, homogeneous nebula, despite small clump-covering factors (Giammanco et al. 2004). Typically, these studies do not resolve the spatial structure of the emitting gas. Observations either integrate all the nebular light and lose all spatial information, or study structure from a single long slit spectrum. By simplifying the line fluxes of an entire $\mathrm{H}$ II region to a single value, valuable information about the true structure of the gas is lost.

The correlation between DIG surface brightness and proximity to $\mathrm{HII}$ regions is another key piece of evidence for the leakage of ionizing radiation from discrete $\mathrm{H}$ II regions and can be used to estimate the optical depth. Seon (2009) used these correlations to test a model of M51 where leaking $\mathrm{H}$ II regions explain the observed DIG and $\mathrm{H} \alpha$ surfacebrightness distributions, similar to the method used by Zurita et al. (2002) in NGC 157. However, Seon (2009) found that this model requires a highly rarefied or porous ISM with an anomalously low dust abundance. These details are inconsistent with the known properties of M51, suggesting that the models do not fully explain the propagation of radiation in real galaxies.

Thus, existing methods to determine nebular optical depth are subject to large uncertainties; clearly it would be preferable to have a diagnostic that is reliable, effective, and simple. Here, we offer such a diagnostic, using an approach that makes it possible to accurately characterize the optical depth of individual H II regions in the nearest galaxies. In Section 2, we describe our method; in Section 3, we apply our technique to the Magellanic Clouds and use it to generate a new, physically motivated H II region catalog; and we evaluate our technique in Section 4. Our results yield powerful new insights on the radiative transfer of LyC radiation from massive stars in these galaxies, which we present in Section 5.

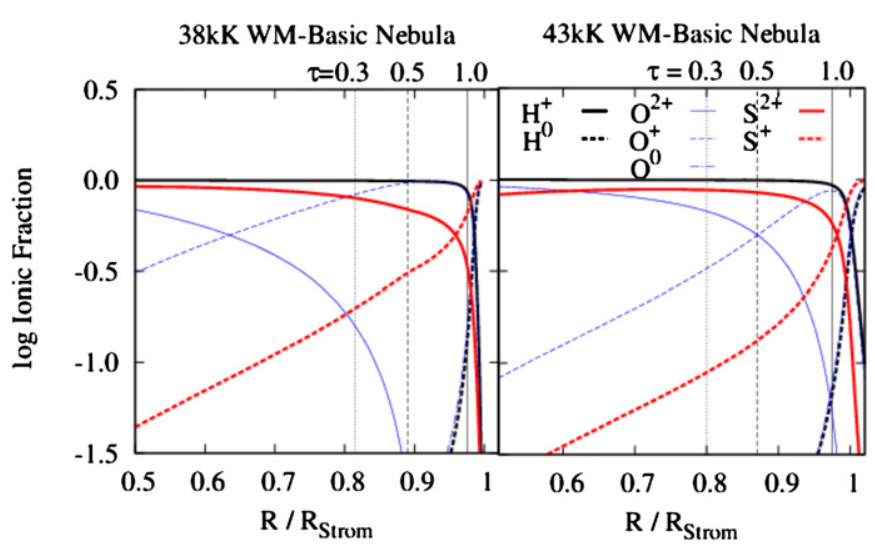

Figure 1. Radial ionization structure for Strömgren sphere H II regions photoionized by a $T_{\text {eff }}=38 \mathrm{kK}$ star (left panel) and a $43 \mathrm{kK}$ star (right panel), assuming a gas density of $10 \mathrm{~cm}^{-3}$. The logarithmic ionization fractions of $\mathrm{H}^{+}$ (solid thick black line), $\mathrm{H}^{0}$ (dashed thick black line), $\mathrm{O}^{2+}$ (solid thin blue line), $\mathrm{O}^{+}$(dashed thin blue line), $\mathrm{O}^{0}$ (dash-dot blue line), $\mathrm{S}^{2+}$ (solid thick red line), and $\mathrm{S}^{+}$(dashed thick red line) are plotted. The vertical lines mark radii where $\tau_{\text {LyC }}=0.3$ (dotted), and 0.5 (dashed) to 1.0 (solid).

(A color version of this figure is available in the online journal.)

\section{IONIZATION-PARAMETER MAPPING}

With the recent availability of wide-field, narrowband imaging and tunable filters, the potential of spatially resolved, emission-line diagnostics as constraints on nebular models is being more fully realized, and these techniques can now be applied to entire populations of extragalactic nebulae. We revisit a largely overlooked approach, ionization-parameter mapping (IPM), which is capable of directly assessing the optical depth of ionizing radiation in individual $\mathrm{H}_{\mathrm{II}}$ regions (e.g., Koeppen 1979). The technique is based on emission-line ratio mapping, which has been previously employed (e.g., Heydari-Malayeri 1981; Pogge 1988a, 1988b); here, we present a modern development, demonstration, and application. The current approach is driven by newly available data with unprecedented sensitivity, resolution, and spatial completeness. We leverage this data against recent developments in the ability to predict spatially resolved, emission-line diagnostics with photoionization models. Our method thus balances the quantitative diagnostics of spectroscopy and the spatial coverage of imaging, yielding a powerful method that is both observationally efficient and straightforward enough to be applied to entire galaxies.

\subsection{Evaluating Nebular Optical Depth}

For classic, optically thick H II regions, there is a transition zone between the central, highly excited region and the neutral environment. These transition zones are characterized by a strong decrease in the excitation, and hence also in the gas ionization parameter, which traces the degree of ionization and photon-to-gas density. Figures 1(a) and (b) show the radial ionic structure of Strömgren spheres generated by a $38,000 \mathrm{~K}$ and a $43,000 \mathrm{~K}$ star, respectively. These demonstrate the transition from highly ionized inner zones dominated by $\mathrm{O}^{2+}$ and $\mathrm{S}^{2+}$ to outer envelopes dominated by $\mathrm{O}^{+}$and $\mathrm{S}^{+}$. The low-ionization transition zone is thicker than the narrow $\mathrm{H}^{0} / \mathrm{H}^{+}$ionization front where the $[\mathrm{S}$ II] volume emissivity peaks (Osterbrock \& Ferland 2006); this results from the sensitivity of the [S II]/[O III] ratio to the radial difference between the $\mathrm{O}^{2+}$ and $\mathrm{H}^{+}$recombination fronts, which are in turn determined by the LyC optical depth $\tau_{\mathrm{LyC}}$ and stellar effective temperature $T_{\text {eff }}$. This largescale gradient is a key feature to the application of IPM at great 


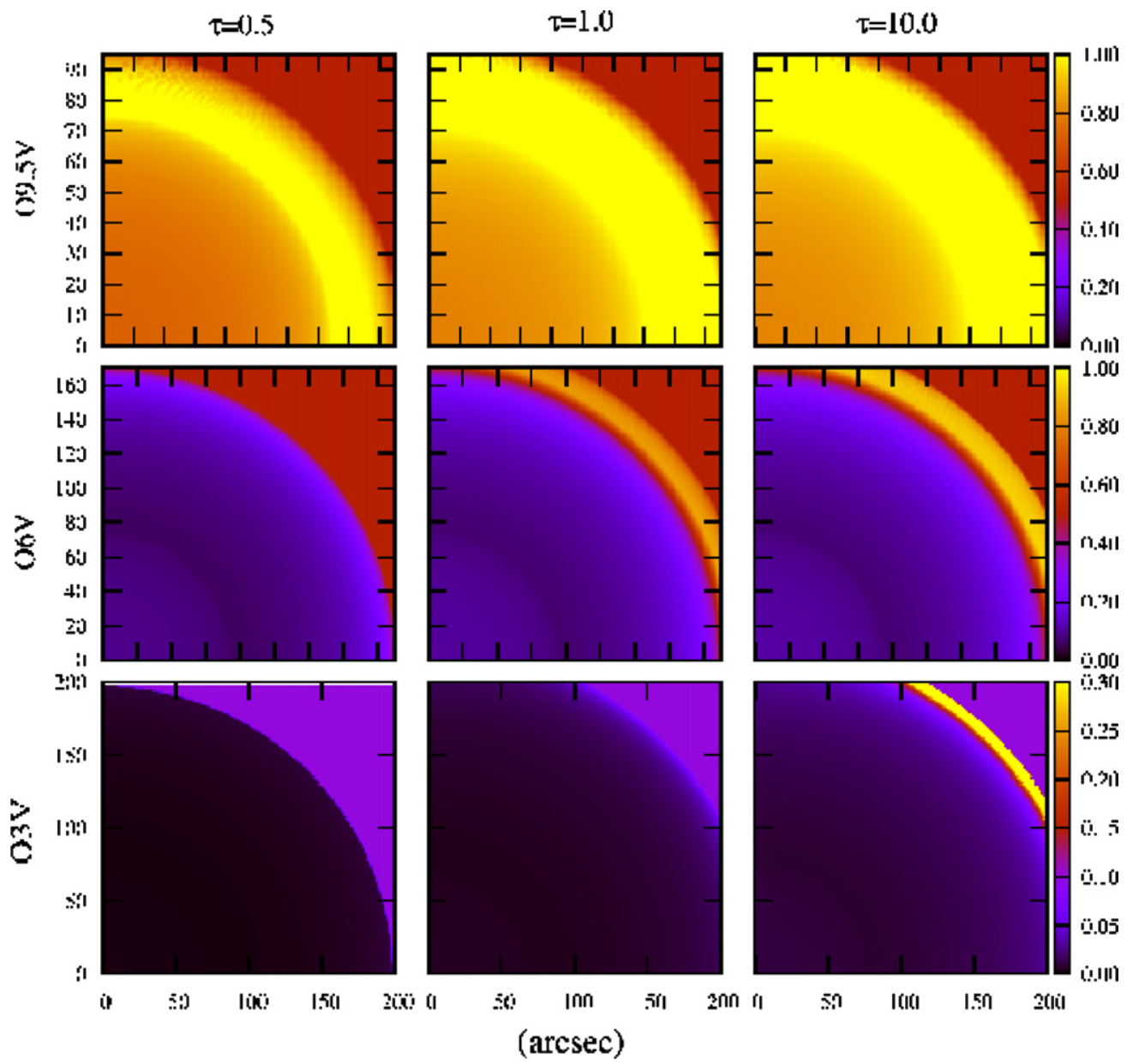

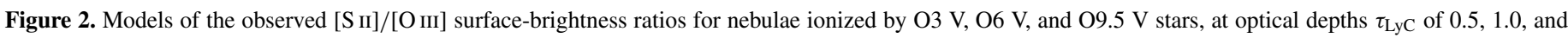
10. A uniform background is assumed, and the units on both axes are in arcseconds, projecting the objects at the LMC distance.

(A color version of this figure is available in the online journal.)

distances. For the models in Figure 1, the assumed ionizing SED is a single WM-Basic stellar atmosphere (Smith et al. 2002) defined by a variable $T_{\text {eff }}$ and fixed $Q\left(\mathrm{H}^{0}\right)=10^{49} \mathrm{~s}^{-1}$, equivalent to one $\mathrm{O} 6 \mathrm{~V}$ star. Calculations were performed using the CLOUDY photoionization code, version C08.00 (Ferland et al. 1998), adopting gas-phase abundances equal to those of the 30 Doradus star-forming region, having $\log (\mathrm{O} / \mathrm{H})=-3.75$ (Pellegrini et al. 2011). Our models include dust with a gas-todust ratio of $A_{\mathrm{V}} / N(\mathrm{H})=1.8 \times 10^{-23} \mathrm{~cm}^{-2}$, which is consistent with the ionized gas studied by Pellegrini et al. (2011). We use dust with a Large Magellanic Cloud (LMC) size distribution described by Weingartner \& Draine (2001), although our results are not sensitive to the dust abundance. The initial $\mathrm{H}$ density $n_{\mathrm{H}}$ is equal to $10 \mathrm{~cm}^{-3}$, and the distance $r_{0}=0.1 \mathrm{pc}$ between the illuminated face of the cloud and the ionizing source. Deeper in the cloud $n_{\mathrm{H}}$ is set by a hydrostatic equation of state with no magnetic field ( $\mathbf{B}=0 \mathrm{G}$ ), described in Pellegrini et al. (2007).

Figure 1 demonstrates that we can estimate $\tau_{\text {LyC }}$ from the observed ion stratification within the nebula, which depends strongly on $\tau_{\text {LyC }}$. While nebulae ionized by different $T_{\text {eff }}$ have greatly differing structure, the optical depth is strongly constrained by the radial structure in two ions, and essentially uniquely determined by three ions. Figure 2 shows models of the observed surface-brightness ratios for the $[\mathrm{S}$ II] $\lambda \lambda 6716,6731$ and [O III] $\lambda 5007$ emission lines for a series of $\mathrm{H}$ II regions with LMC element abundances. We calculate the projected two- dimensional (2D) surface brightness of our models according to Equation (2) of Pellegrini et al. (2009) using the SurfBright routine, which is described in Appendix A. We have added a constant, noiseless background of $1 \times 10^{-15} \mathrm{erg} \mathrm{s}^{-1} \mathrm{~cm}^{-2} \operatorname{arcsec}^{-2}$, consistent with typical $\mathrm{H}$ II region observations. We note that decreasing the background component will enhance the predicted contrast, while an increase reduces contrast. The model parameters of these simulations are similar to those of the models in Figure 1. The nebulae are ionized by a single WM-Basic (Smith et al. 2002) stellar SED with an ionizing luminosity equivalent to a cluster of $10 \mathrm{O} 6 \mathrm{~V}$ stars, and $T_{\text {eff }}$ equal to $30,500 \mathrm{~K}, 38,000 \mathrm{~K}$, or $44,500 \mathrm{~K}$. These $T_{\text {eff }}$ correspond to $09.5 \mathrm{~V}$, O6 V, and O3 V spectral types, respectively, using the spectral-type- $T_{\text {eff }}$ calibration of Martins et al. (2005). A single $T_{\text {eff }}$ is often used to represent the SED of ionizing clusters, which is a reasonable approximation since the earliest spectral type dominates the SED (e.g., Oey \& Shields 2000). Figure 2 shows models for $\tau_{\mathrm{LyC}}=0.5,1.0$, and 10.0 , at each $T_{\mathrm{eff}}$, where the cloud is truncated at various radii to simulate the different $\tau_{\mathrm{LyC}}$.

Figure 2 demonstrates how the optical depth and $T_{\text {eff }}$ determine the observed ionic structure that is rendered by IPM. In general, the $\tau_{\mathrm{LyC}}=0.5$ models show no low-ionization transition layer, although there is an exception for the latest spectral type. For early- and mid-O spectral types, the morphology in these ionization-parameter maps is an especially strong discriminant for the optical depth. And as shown in Figure $1, \tau_{\mathrm{LyC}}$ can 


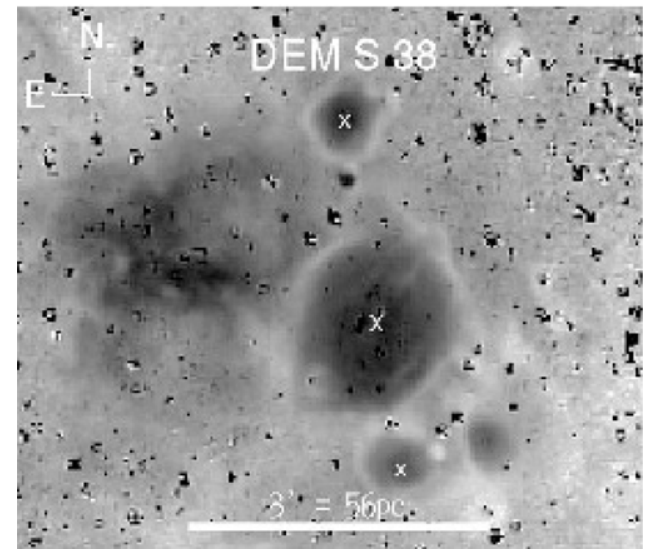

Figure 3. Map of the $[\mathrm{S}$ II $] /[\mathrm{O}$ III] ratio centered on $\mathrm{H}$ II region DEM S38. The central region is a classic example of an optically thick nebula with highly ionized gas (low [S II]/[O III]; dark) surrounded by an ionization transition zone with higher $[\mathrm{S} \mathrm{II}] /[\mathrm{O} \mathrm{III}]$ (lighter gray scale). The same effect is seen in the regions to the north and south of DEM S38, which are marked with crosses. In contrast, the irregular H II region seen to the east (left) of DEM S38 shows no evidence of such a transition zone between the high-ionization region and the galactic background, indicating that it is optically thin.

be fully constrained when surface-brightness ratios are obtained for three radially varying ions instead of two. We further discuss the use and limitations of our method in Section 2.2 below.

Figure 3 shows the observed ratio map of $[\mathrm{S} \mathrm{II}] /[\mathrm{O} \mathrm{III}]$ for a star-forming complex centered on the nebula DEM S38, from the Magellanic Clouds Emission Line Survey (MCELS; Smith et al. 1998, 2005; Points et al. 2005; Winkler et al. 2005). We clearly see an envelope of low-ionization gas surrounding a high-excitation interior in each $\mathrm{H}$ II region marked with an $\mathrm{X}$ (DEM S38 and the two regions to the north and south), strongly suggesting that these objects are optically thick. In contrast, the nebula east of DEM S38 shows high ionization throughout and no evidence of an internal gradient in gas ionization state. This indicates that the object is optically thin.

We also see that the object DEM S159 (Figure 4) shows the intermediate morphology of a blister-like H II region. Like DEM S38, there is a central region of highly ionized gas, but a transition zone of weakly ionized gas is found only to the north, while toward the south, the nebula remains highly ionized throughout, like our $\tau_{\mathrm{LyC}}=0.5$ models in Figure 2. Since all of the nebula is ionized by the same SED, DEM S159 must be optically thick to the north and optically thin to the south. Thus, Figures 3 and 4 vividly demonstrate the viability of IPM as a technique to evaluate $\tau_{\mathrm{LyC}}$. The morphology of the ionization structure in these objects is qualitatively consistent with our models, and in Section 4 below, we also show quantitatively that observations are consistent with predictions.

Furthermore, the contrasting gas morphology between the spherical, optically thick nebulae and the irregular, optically thin object in Figure 3 is not a coincidence. In the MCELS data for the Magellanic Clouds, most of the optically thick objects showing low-ionization envelopes look like classical, spherical, Strömgren spheres. The opposite is true for optically thin objects, which are more complex and irregular in morphology. This is consistent with recent radiation-MHD simulations by Arthur et al. (2011), which show that the highly ionized, density-bounded nebulae powered by the hottest stars are subject to strong radiative feedback and gas instabilities, generating irregular gas morphologies. Thus, the gas morphologies are fully consistent with the interpretation that objects having low-

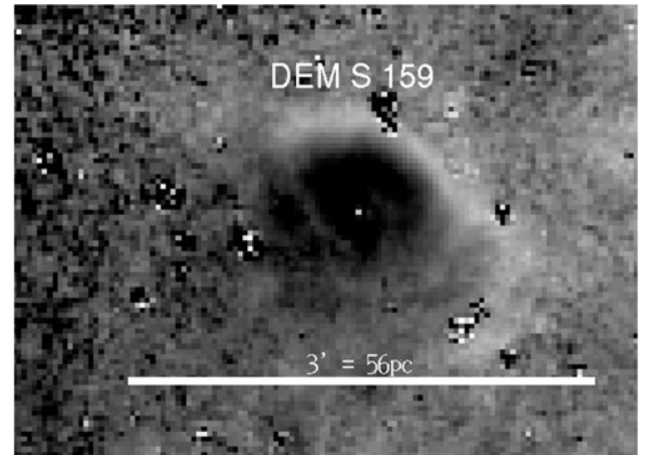

Figure 4. DEM S159 shows blister-like features, exhibiting nebular traits for both high and low optical depths. As in Figure 3, the ratio of [S II]/[O III] reveals the presence of highly ionized gas (dark) in this region. This is confined to the northwest by a pronounced ionization transition zone, but not to the southeast.

ionization transition zones are generally optically thick and radiation bounded.

Finally, IPM also constrains the optical depth in the line of sight, since the low-ionization transition zone should also exist along these photon paths in an optically thick nebula. This was explored by Pellegrini et al. (2011) who found a lower limit of $[\mathrm{S} \mathrm{II}] / \mathrm{H} \alpha \sim 0.05$ for LMC nebulae that are optically thick in the line of sight. Lower values of $[\mathrm{S} \mathrm{II}] / \mathrm{H} \alpha$ indicate that the low-ionization transition zone is missing or depleted, and therefore that the region is optically thin to the LyC. In Figure 5 we compare the line-of-sight emission-line ratios of DEM S15 and N59, a pair of optically thick and optically thin H II regions. DEM S15 is a classical Strömgren sphere, limb brightened in the lower ionization species, and it shows a central line-of-sight $[\mathrm{S}$ II $] / \mathrm{H} \alpha=0.05$, consistent with an optically thick nebula of Small Magellanic Cloud (SMC) metallicity. In contrast, in N59, the central $[\mathrm{S} \mathrm{II}] / \mathrm{H} \alpha$ ratio is essentially zero across much of the object, and thus no transition zone is seen in those sight lines, demonstrating that the object is optically thin.

\subsection{Limitations for Two-ion Mapping}

IPM is tremendously powerful and can even be done with only two radially varying ions. When using only two ions, we caution that the technique has three limitations. Ostensibly, the most important quantity to be derived with this technique is the escape fraction of LyC photons from an individual $\mathrm{H}$ II region, $f_{\text {esc }}$, defined as

$$
f_{\mathrm{esc}}=e^{-\tau_{\mathrm{LyC}}} .
$$

IPM based on only two ions can provide only lower limits on $f_{\text {esc }}$ because the observed morphology becomes degenerate at high $f_{\text {esc }}$. This can be seen in the bottom row of Figure 2 for $\tau_{\text {LyC }} \leqslant 1.0$, which corresponds to $f_{\text {esc }} \geqslant 40 \%$. In these cases, background emission masks the very faint, lower-ionization emission lines in fully ionized gas, and the ratio ceases to directly track changes in the $\mathrm{H}$ II region ionization structure. This problem worsens as $T_{\text {eff }}$ increases, and it becomes more difficult to identify the transition to neutral gas. However, only the hottest ionizing stars in the local universe will have $T_{\text {eff }} \sim 44,500 \mathrm{~K}$, and when three ions are available, the degeneracies are resolved.

There is also a degeneracy between optically thin nebulae ionized by cool stars $\left(T_{\text {eff }} \lesssim 34,000 \mathrm{~K}\right)$ and optically thick regions heated by hotter stars. The degeneracy exists where cool stars do not emit much radiation above $35 \mathrm{eV}$ to generate $\mathrm{O}^{2+}$, and so these nebulae are entirely dominated by $\mathrm{O}^{+}$. Again, IPM based on three ions, adding $\mathrm{S}^{2+}$ for example, can 


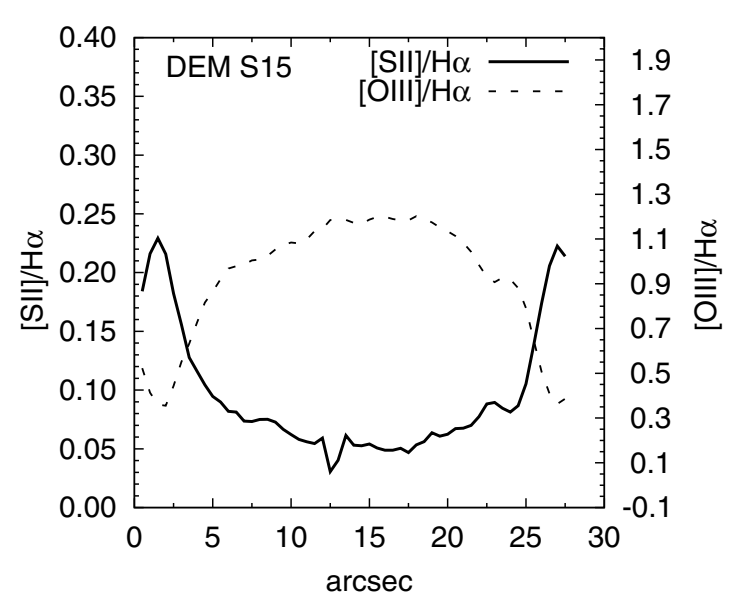

(a) DEM S15

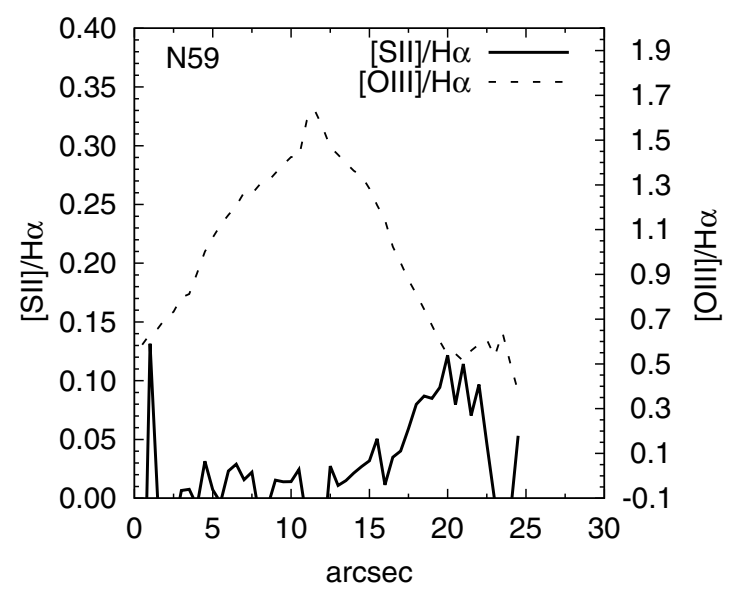

(b) N59

Figure 5. Spatial profiles of $\left[\mathrm{O}_{\mathrm{III}}\right] / \mathrm{H} \alpha$ and $\left[\mathrm{S}_{\mathrm{II}}\right] / \mathrm{H} \alpha$ across the center of the optically thick object DEM S15 (a) and optically thin region N59 (b).

resolve the degeneracy (Figure 1). However, we stress that this problem applies primarily to the lowest-luminosity objects, and as we show below, their aggregate luminosity is insignificant compared to the total amount of energy found to be escaping all $\mathrm{H}$ iI regions.

Finally, we again caution that for a population of randomly oriented blister $\mathrm{H}$ II regions, it is likely that the orientation of some objects will cause a projected ionization-parameter gradient that appears optically thick on the limb, but is optically thin in the line of sight. The most extreme example is of a halfsphere, blister nebula viewed directly face-on: despite having $f_{\text {esc }}=0.5$, the projected region is circular and will show an ionization transition zone associated with the optically thick half. However, as discussed in Section 2.1, the ionic ratios of [S II], [O III], and $\mathrm{H} \alpha$ across the central region of these nebulae should show a deficit in lower-ionization species that is incompatible with optically thick models (Figure 5(b)). With further constraints on the ionizing SED and a quantitative evaluation of these ratios, we can still measure their optical depth. Thus, there may be instances where optically thin H II regions are initially misidentified as optically thick, but these can be identified by quantitative examination of spatially resolved ionic ratios. If objects are misidentified, this again would favor underestimates of $\tau_{\mathrm{LyC}}$.

Hence, the caveats identified above can be resolved by IPM in three ions and quantitative evaluation of the entire nebular projection. We note that these fairly manageable issues all work to underestimate the optical depth of $\mathrm{H}$ II regions. Thus, IPM shows great promise as a powerful tool in studies of the ISM. Emission-line ratio maps neutralize variations in surface brightness, clearly revealing changes in ionization structure for bright and faint regions alike. The power of the technique is that it allows us to identify optically thin $\mathrm{H}$ II regions by the absence of the low-ionization envelope, which almost always indicates that the nebula is density bounded.

\section{IONIZATION-PARAMETER MAPPING OF THE LMC AND SMC}

We now apply our technique of IPM to the LMC and SMC, which have been mapped with narrowband emission-line imaging by the MCELS survey. This is a spatially complete, fluxlimited survey carried out at the Cerro Tololo Inter-American Observatory (CTIO) with the University of Michigan's Curtis $0.6 / 0.9 \mathrm{~m}$ Schmidt telescope. Over the course of five years, the LMC and SMC were imaged in [S II] $\lambda \lambda 6717,6731$, [O III] $\lambda 5007$, and $\mathrm{H} \alpha$, with respective filter widths of 50,40 , and $30 \AA$. The $\mathrm{H} \alpha$ filter bandpass includes [N II] $\lambda \lambda 6548,6584$ at a reduced throughput. The final product, mosaics in both low- and high-ionization line emission, and in the $\mathrm{H} \alpha$ recombination line, traces the ionized ISM at both large and small scales. The process of mosaicking the images resulted in a binned pixel scale of 3.0 and 2.0 arcsec pixel $^{-1}$ for the LMC and SMC, respectively. These correspond to a spatial scale of $0.7 \mathrm{pc}$ and $0.6 \mathrm{pc}$ for distances of $49 \mathrm{kpc}$ (Macri et al. 2006) and $61 \mathrm{kpc}$ (Hilditch et al. 2005), respectively, with an effective resolution of $\sim 5$ arcsec. The $1 \sigma$ surface-brightness limit of each band is listed in Table 1. These are the sensitivities per pixel, expressed as surface brightness in erg s $\mathrm{s}^{-1} \mathrm{~cm}^{-2} \operatorname{arcsec}^{-2}$ and $\mathrm{H} \alpha$ emission measure (EM) in $\mathrm{pc} \mathrm{cm}^{-6}$. Such depth is important to form a complete understanding of the WIM ionization, and the dependence of $f_{\text {esc }}$ on star formation intensity and $\mathrm{H}$ II region properties.

The MCELS survey includes continuum observations centered at $5130 \AA$ and $6850 \AA$, with effective bandpasses of $155 \AA$ and $95 \AA$, respectively. These were used to produce a continuumsubtracted mosaic of the SMC (Winkler et al. 2005). Based on spectrophotometric observations of the SMC region NGC 346 by Tsamis et al. (2003), we estimate that the flux calibration of the continuum-subtracted data has uncertainties on the order of $20 \%$. At present, the LMC data are not yet continuum subtracted. To flux-calibrate the LMC data, we used spectrophotometric observations by Pellegrini et al. (2010), extracting MCELS line fluxes along the length of slit position 5 in that paper to determine the flux constants. We also compare against the flux-calibrated, narrowband data obtained on the SOAR telescope in a 30 arcsec circular aperture at the position $\alpha=$ 05:38:56.9, $\delta=-69: 05: 21.8$ (J2000) (Pellegrini et al. 2010). We find that the comparisons agree within approximately $20 \%$, which then correspond to the systematic uncertainty in our flux calibration.

We generated line-ratio maps of $[\mathrm{S} \mathrm{II}] /[\mathrm{O} \mathrm{III}]$ for both the LMC and SMC using IRAF, ${ }^{4}$ with the LMC maps based on non-continuum-subtracted emission-line images and the SMC maps based on continuum-subtracted images. These ratio maps, which probe the ionization parameter in the ionized gas, are shown in Figures 6 and 7, respectively.

\footnotetext{
4 IRAF is distributed by the National Optical Astronomy Observatory, which is operated by the Association of Universities for Research in Astronomy (AURA) under cooperative agreement with the National Science Foundation.
} 


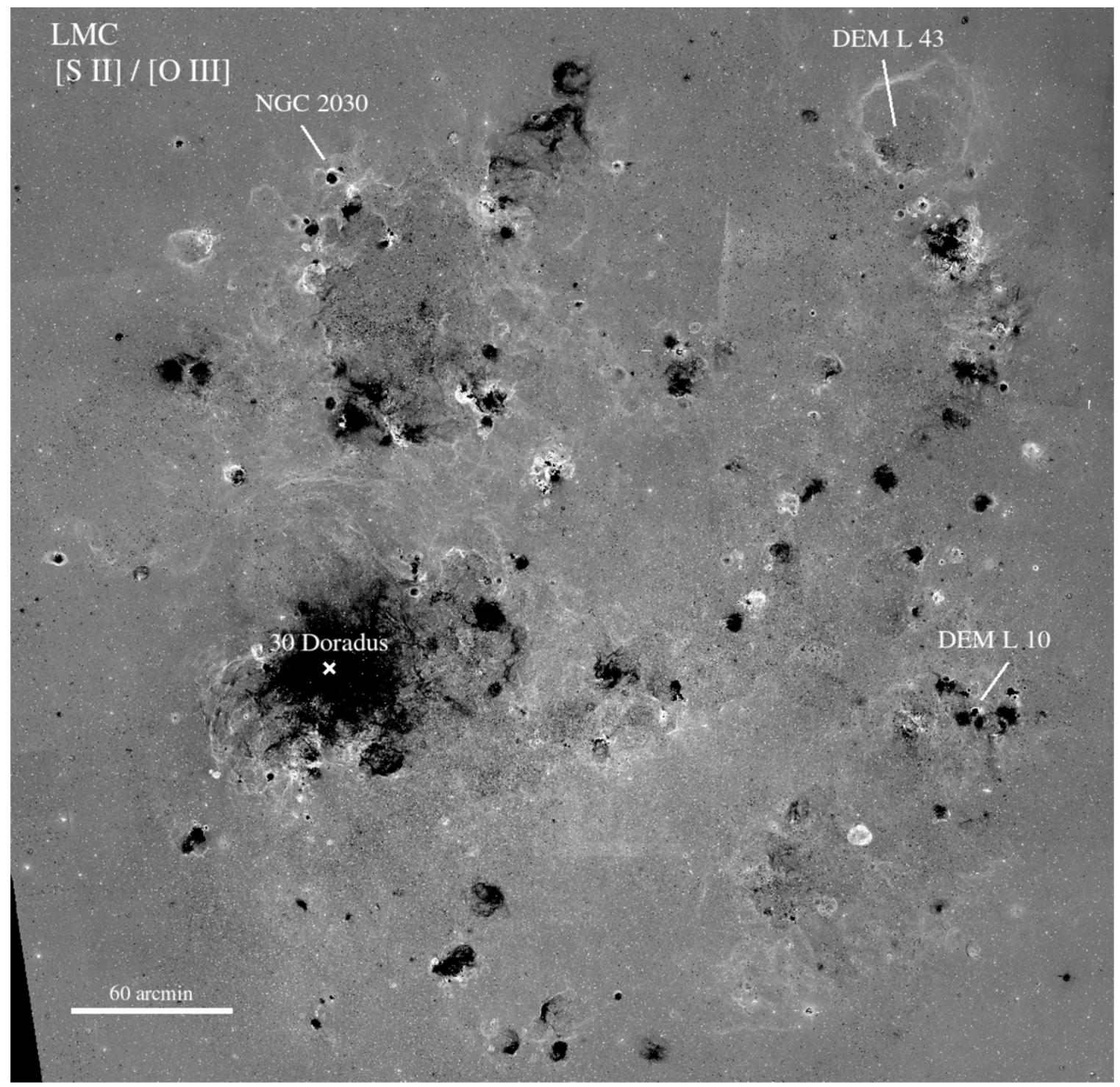

Figure 6. Map of $\log \left[\mathrm{S}_{\mathrm{II}}\right] /[\mathrm{O}$ III $]$ for the entire LMC galaxy created from the MCELS narrowband imaging data. Black corresponds to low [S II $] /[\mathrm{O}$ III $]$; this ratio map is not based on continuum-subtracted data. North is up, east is to the left in the center of the field.

Table 1

MCELS $1 \sigma$ Surface Brightness Detection Limits

\begin{tabular}{lcccc}
\hline \hline Galaxy & $\begin{array}{c}S(\mathrm{H} \alpha) \\
\left(\mathrm{erg} \mathrm{s}^{-1} \mathrm{~cm}^{-2} \operatorname{arcsec}^{-2}\right)\end{array}$ & $\begin{array}{c}\mathrm{EM}(\mathrm{H} \alpha) \\
\left(\mathrm{pc} \mathrm{cm}^{-6}\right)\end{array}$ & $\begin{array}{c}S([\mathrm{O} \text { III] }) \\
\left(\mathrm{erg} \mathrm{s}^{-1} \mathrm{~cm}^{-2} \operatorname{arcsec}^{-2}\right)\end{array}$ & $\begin{array}{c}S[\mathrm{~S} \mathrm{II}]) \\
\left(\mathrm{erg} \mathrm{s}^{-2} \operatorname{arcsec}^{-2}\right)\end{array}$ \\
\hline LMC & $7.0 \mathrm{E}-18$ & 3.5 & $1.4 \mathrm{E}-17$ & $5.2 \mathrm{E}-18$ \\
SMC & $7.2 \mathrm{E}-18$ & 3.6 & $5.4 \mathrm{E}-17$ & $1.0 \mathrm{E}-17$ \\
\hline
\end{tabular}

Notes. Values shown are the $1 \sigma$ uncertainties in a single pixel for surface brightness and emission measure as shown. The LMC data are not continuum subtracted and have a pixel scale of 3 arcsec, while the SMC data are continuum subtracted and have a pixel scale of 2 arcsec.

\subsection{Ionization-based H II Region Catalogs}

IPM allows us to assign physically motivated H II region boundaries in complex, confused regions with multiple ionizing sources. In areas where $\mathrm{H}$ II regions are overlapping, or are found in complex ionized backgrounds, the ionization stratification makes it possible to isolate individual photoionized regions, which is impossible with imaging in only $\mathrm{H} \alpha$ or any single line. In particular, IPM allows us to define nebular boundaries based on both ionization structure and $\mathrm{H} \alpha$ surface brightness $S(\mathrm{H} \alpha)$ morphology. The examples in Figure 8 demonstrate how IPM generates contrast between the DIG and low surface brightness, extended $\mathrm{H}$ II regions that are independently ionized entities. In the $\mathrm{H} \alpha$ image (right panel of Figure 8), the objects DEM S10 and DEM S49 are amorphous regions that blend into the surrounding DIG with ambiguous boundaries. In contrast, the [S II]/[O III] ratio map clearly shows them as distinct regions. The boundaries of optically thick objects are usually unambiguous because these are characterized by a stratified ionization structure as described above, accompanied by a sharp decrease in surface brightness.

Since previous nebular catalogs for the Magellanic Clouds are based only on $\mathrm{H} \alpha$ morphology (e.g., Henize 1956; Davies 


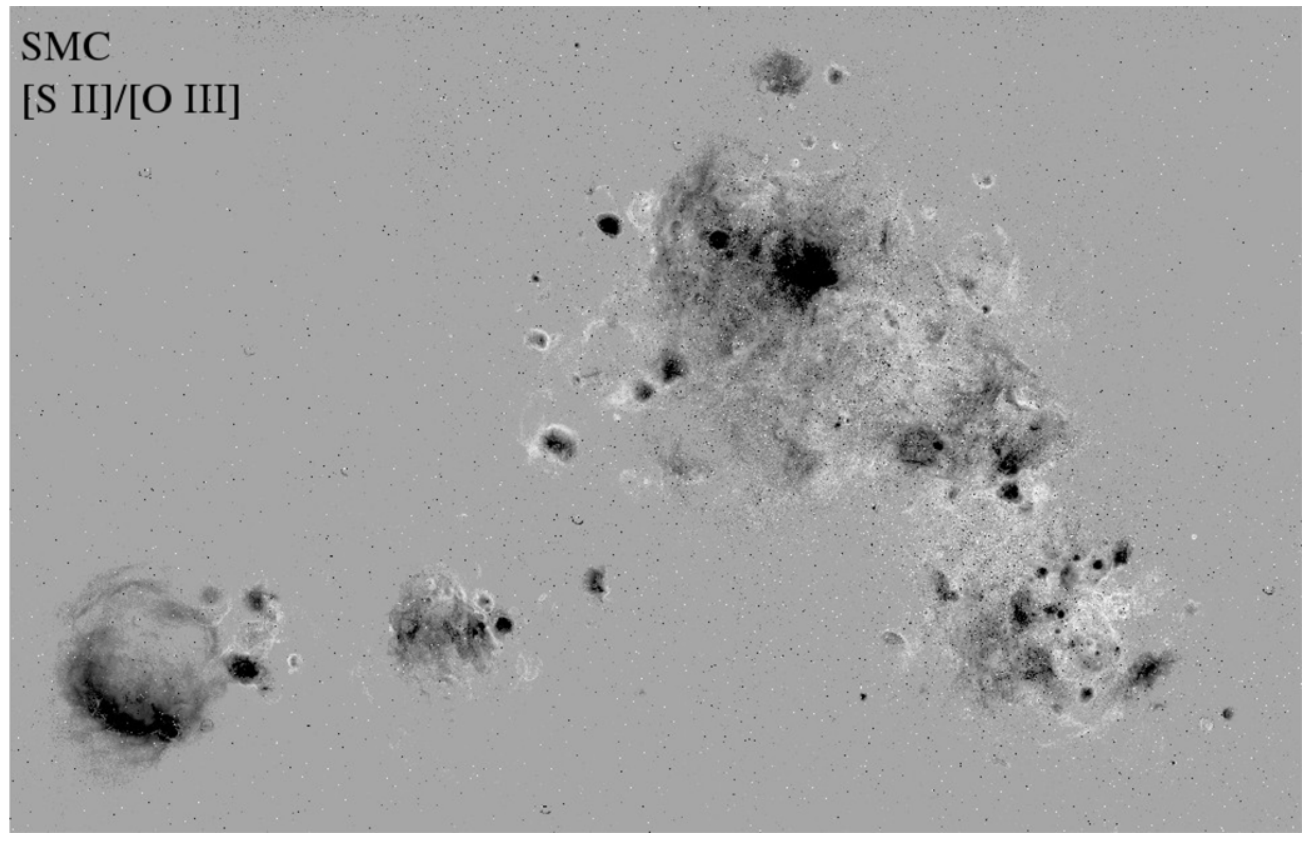

Figure 7. Same as Figure 6 for the SMC galaxy. This ratio map is based on continuum-subtracted data.
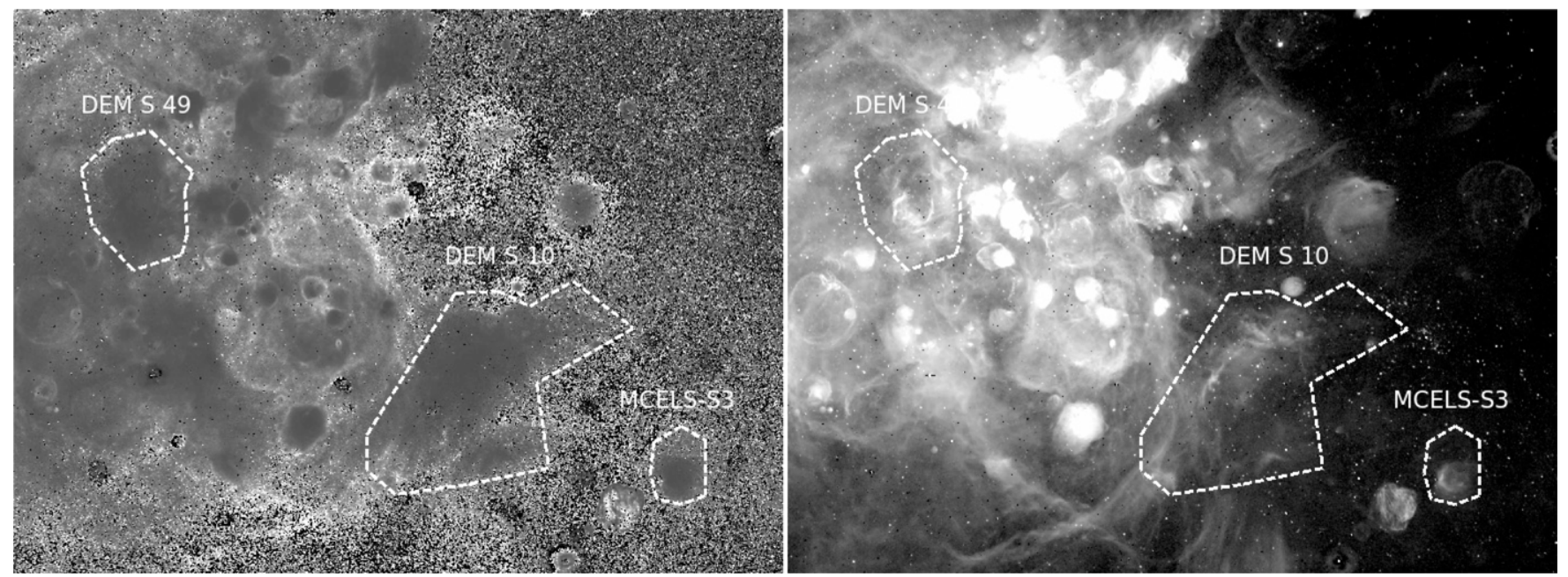

Figure 8. $\left[\mathrm{S}_{\mathrm{II}}\right] /\left[\mathrm{O}_{\mathrm{III}}\right]$ ratio map (left) and $\mathrm{H} \alpha$ (right) for an $\mathrm{SMC}$ region, demonstrating the advantage of using $\left[\mathrm{S}_{\mathrm{II}}\right] /\left[\mathrm{O}_{\mathrm{III}}\right]$ in combination with $\mathrm{H} \alpha$ to define the boundaries of extended H II regions coincident with complex background structures (e.g., DEM S49), and those with extended, faint emission (e.g., DEM S10, MCELS-S3). We indicate H II region boundaries for DEM S10, DEM S49, and MCELS-S3 with dashed lines in both images.

et al. 1976), we use these more sophisticated criteria based on IPM to compile a more physically based catalog of $\mathrm{H}$ II regions in these galaxies. In the case of extended, optically thin objects that show only gradual changes in $[\mathrm{S}$ II $] / \mathrm{H} \alpha$ or $[\mathrm{S}$ II $] /[\mathrm{O}$ III $]$, we define the $\mathrm{H}$ II region boundary to be the point at which either the $S(\mathrm{H} \alpha)$ or the ionic ratio becomes indistinguishable from the DIG, whichever is larger in size. We defined photometric apertures with polygons in SAOImage DS9, for both target objects and local background regions; we used the FUNCNTS routine from FUNTOOLS ${ }^{5}$ to measure the fluxes.

The photometry of faint objects, especially seen in [S II] and [O III] filters, are at risk of being contaminated by stellar continuum in the LMC, where our data are not continuum subtracted. We minimize this contamination by avoiding foreground Galactic stars and also sampling the local density of field stars with our background apertures. Despite the careful creation of apertures,

\footnotetext{
5 https://www.cfa.harvard.edu/ john/funtools/
}

the difference between stellar populations inside and outside the $\mathrm{H}$ II regions may still introduce significant errors, since the most massive, brightest stars often reside within $\mathrm{H}$ II regions. Thus, errors in the background subtraction dominate the flux uncertainties for both galaxies, and they are largest for low surface brightness objects. We therefore find that the median local background surface brightness for nebulae in the LMC is larger than for the SMC: $7.0 \times 10^{-16}$ and $1.3 \times 10^{-16} \mathrm{erg} \mathrm{s}^{-1} \mathrm{~cm}^{-2} \operatorname{arcsec}^{-2}$, respectively, in $\mathrm{H} \alpha$. However, we stress that high surface brightness emission dominates most objects, yielding median background uncertainties of $6 \%$ and $8 \%$ in the LMC and SMC, respectively. For the LMC, the discrete stellar contributions can increase this uncertainty to about $18 \%$. This is consistent with a comparison of our background-subtracted fluxes of bright, isolated LMC and SMC H II regions to their fluxes reported by Kennicutt et al. (1989). We find that the independent measurements agree within $20 \%$, which now also include systematic uncertainties. 


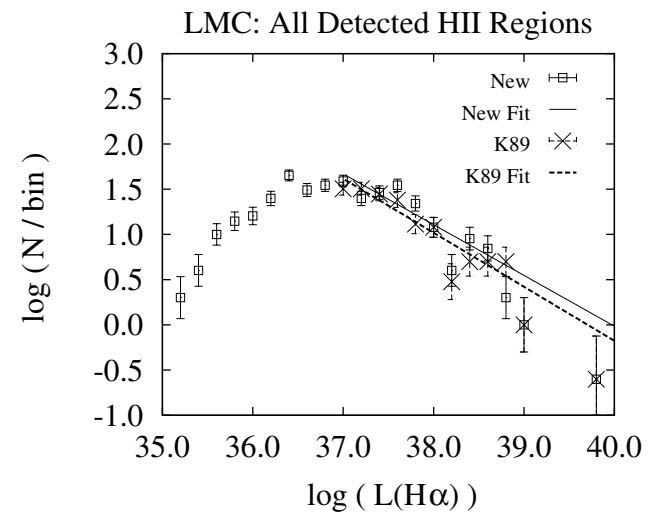

(a) LMC

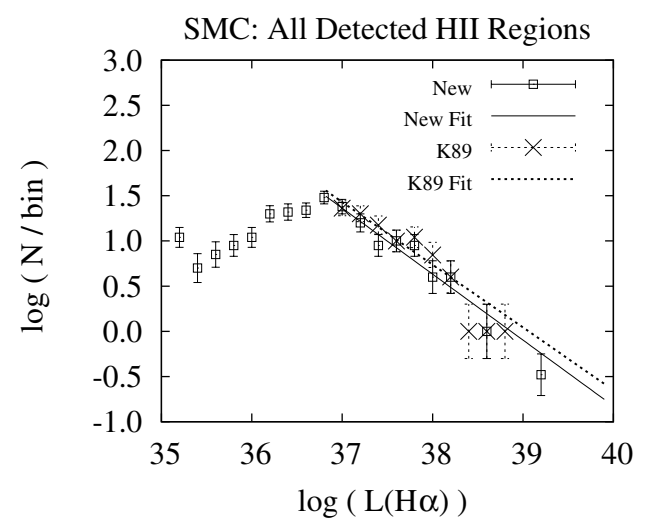

(b) SMC

Figure 9. LMC (a) and SMC (b) H II LFs for our catalogs of photoionized H II regions (squares), and from Kennicutt et al. (1989; crosses). Power-law fits to the data, weighted by inverse error, are shown with the solid and dotted lines, respectively, for our data and those of Kennicutt et al. The error bars show the root- $N$ uncertainties.

We further explored the limiting case of applying a constant background to all objects. For the LMC, we calculated this background from the mean of three locations, two in the north and one in the south to estimate the contamination from sources producing a constant background such as the sky, large-scale diffuse emission, etc. We find the mean, constant background $S(\mathrm{H} \alpha)=$ $5.2 \times 10^{-16} \mathrm{erg} \mathrm{s}^{-1} \mathrm{~cm}^{-2} \operatorname{arcsec}^{-2}$. For the SMC, we determined the background of the continuum-subtracted $\mathrm{H} \alpha$ image to be consistent with zero $\left(7 \times 10^{-18} \pm 7 \mathrm{erg} \mathrm{s}^{-1} \mathrm{~cm}^{-2} \operatorname{arcsec}^{-2}\right.$; cf. Table 1). For both galaxies, subtracting the median backgrounds affects the resulting $L$ by no more than 0.2 dex and does not substantively change our results.

Our H II region catalogs for the LMC and SMC defined with these ionization-based criteria are presented in Appendix B, Tables 5 and 6, which give luminosities and associated $\mathrm{H}$ I column densities for 401 objects in the LMC and 214 in the SMC.

\subsection{The H II Region Luminosity Function}

We find that our $\mathrm{H}$ II region boundaries and $\mathrm{H} \alpha$ luminosities generally agree with those determined in previous, $\mathrm{H} \alpha$-only studies by, e.g., Kennicutt et al. (1989), including the substructure in most of the DEM (Davies et al. 1976) and Henize (1956) surveys. This is especially true for simple objects with a low local background. Figures 9(a) and (b) show the differential H II region luminosity functions (H II LF) for our new LMC and SMC catalogs, respectively (squares), together with those generated by Kennicutt et al. (1989; crosses), fitted above $\log L=37.0$ (where not explicitly stated, the units of $L$ are $\operatorname{erg~s}^{-1}$ ). The power-law slope of the LMC H II LF reported by Kennicutt et al. is $B=1.75 \pm 0.15$, where

$$
d N(L) \propto L^{-B} d(L) .
$$

This is statistically consistent with the fitted H II LF slope for our data, $B=1.79 \pm 0.08$. An identical analysis for the SMC, as shown in Figure 9, yields an H II LF slope of $B=1.88 \pm 0.09$, for our new catalog, compared to a reported slope of 1.9 from Kennicutt et al. (1989). Thus, although previous measurements of the H II LF do not use our ionization-based criteria, they result in essentially identical LF slopes.

Both Magellanic Cloud H II LF slopes flatten around $\log L=$ 37.0, which is equivalent to $Q\left(\mathrm{H}^{0}\right)=48.9 \mathrm{~s}^{-1}$. This flattening is observed in other galaxies whose $\mathrm{H}$ II LFs probe $\log L<36.0$, including the Milky Way (Paladini et al. 2009), M51 (Lee et al. 2011), and M31 (Azimlu et al. 2011). The observed flattening of the HiI LF in this regime was predicted in Monte Carlo simulations by Oey \& Clarke (1998) and Thilker et al. (2002), and it is caused by stochastic ionizing populations at these low luminosities. For comparison, $\log L=37.0$ is the luminosity of the Orion Nebula, whose parent ionizing cluster has a mass of $4500 \mathcal{M}_{\odot}$ (Hillenbrand \& Hartmann 1998), dominated by a single $06.5 \mathrm{~V}$ star.

A final caveat: at the lowest luminosities, there is a decrease in the H II LFs. This is clearly established for $\log L<36.5$ in both galaxies. The drop in source counts could be an indication that below this $L$ we are not complete. Alternatively, since the stellar ionizing fluxes plummet strongly for stars later than early B spectral types, this turnover in the H II LF signals an intrinsically different class of ionizing sources and nebular objects. These must include individual $\mathrm{H}$ II regions of later B-type stars, and perhaps some faint, optically thin nebulae that are intrinsically weak in recombination lines due to their low optical depth. There also may be some shock-heated filaments, although we tried to avoid most of these. Planetary nebulae should not be important above $L \sim 5 \mathrm{e}^{2} \mathrm{erg} \mathrm{s}^{-1}$ (Azimlu et al. 2011).

\subsection{An Enigmatic, Highly Ionized Region}

The technique of IPM is effective at highlighting large, extremely faint structures. The $[\mathrm{S} \mathrm{II}] /[\mathrm{O} \mathrm{III}]$ ratio map of an LMC object at $\alpha=04: 55: 50, \delta=67: 30: 50$ (J2000) is shown in the left panel of Figure 10, with an inner ellipse to mark the extent of highly ionized and filamentary gas. The middle panel shows a larger contour that highlights an $\mathrm{H}$ i cavity seen in the $\mathrm{H}$ I data of Kim et al. (2003) with a major axis of $550 \mathrm{pc}$ and $\mathrm{H} \mathrm{I}$ column density $N(\mathrm{HI})=0.6 \times 10^{21} \mathrm{~cm}^{-2}$. Both ellipses have the same orientation, suggesting that they are related. The right panel of Figure 10 shows that faint $\mathrm{H} \alpha$ emission is co-spatial with the [O III], while no [S II] was detected. This indicates that the optically emitting gas is fully ionized. This structure is intriguing because the $[\mathrm{S} \mathrm{II}] /[\mathrm{O} \mathrm{III}]$ morphology is similar to optically thin nebulae ionized by $\mathrm{OB}$ stars, yet no ionization sources are known in the region, nor is there evidence of a prior supernova or shocked gas. The size and faintness of this highly excited region, together with the lack of an ionization source, make this object unique. Further observations to identify its nature and origin are required.

\section{OPTICAL DEPTH OF THE H II REGIONS}

From the diagnostics based on ionization structure as described in Section 2, we classify the optical depth of our 


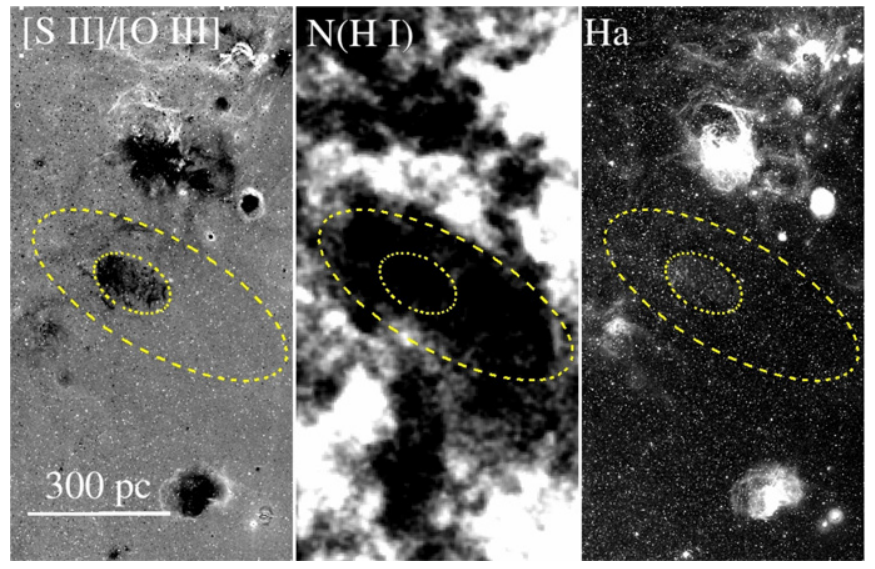

Figure 10. Large, highly ionized bubble with no known ionization source. Left: [S II]/[O III] ratio map; center: $\mathrm{H}_{\mathrm{I}}$ column density map of the region, with white showing $\mathrm{H}_{\mathrm{I}}$ emission; right: the region in $\mathrm{H} \alpha$. All panels are shown with the same scale, where north is up and east is left. The central contour marks the boundary where the object is indistinguishable from the background, while the larger contour marks the rim of the bubble seen in $N(\mathrm{HI})$.

(A color version of this figure is available in the online journal.)

individual, cataloged $\mathrm{H}$ II regions into the following categories: 0 -indeterminate, 1 -optically thick, 2-blister, 3-optically thin, and 4-shocked nebulae. These are given in Column 4 of Tables 5 and 6 . Class 0 objects, with indeterminate optical depth, fall into two categories: those which lack [O III] emission, causing a high $[\mathrm{S} \mathrm{II}] /[\mathrm{O} \mathrm{III}]$ ratio, with little ionization structure; and large-scale, diffuse structures. This latter category is difficult to define morphologically, but since many objects in the DEM LMC catalog include these features, we have attempted to catalog them as well.

We define optically thick objects (class 1) to be those showing classic, low-ionization envelopes enclosing at least two-thirds of the central, high-ionization regions in projection, as described in Section 2. Blister nebulae (class 2) are defined by a low-ionization envelope that surrounds between one-third and two-thirds of the observed object; additionally, objects having complex internal ionization fronts with extended [O III] emission are treated as blisters. Optically thin (class 3) objects show low [S II]/[O III] throughout, with low-ionization envelopes covering $<1 / 3$ of the highly ionized gas (see Figures 3 and 4). Shocked objects (class 4) are characterized by an ionization structure which is inverted relative to photoionization, i.e., these objects have enhanced [O III] emission surrounding strong [S II]. Our survey is not intended to be complete with respect to shocked objects, and we typically avoided cataloguing them. Since these are not photoionized, they are excluded from further consideration. Additional general classification criteria include gradients in ionization parameter, the detection of ionization fronts distinct from the background, and the ionized extent of the object. Radial projections of the $[\mathrm{S}$ II $] /[\mathrm{O}$ III $]$ ratio were made to assess the significance of specific individual features that were identified. Three of the authors (E.W.P., J.Z., and A.E.J.) used these criteria to carry out independent classifications of all the objects. To arrive at a final catalog, we resolved the differences by discussing specific key features and quantitatively measuring the optically thin covering fraction.

We roughly estimate the LyC escape fraction $f_{\text {esc }}$ for each object in classes 1-3 as follows: optically thick objects are assigned $f_{\text {esc }}=0$; optically thin objects are assigned $\tau_{\text {LyC }}=0.5$, which corresponds to $f_{\text {esc }}=0.6$; and blister objects are assigned
Table 2

Comparison of $f_{\text {esc }}$ Estimates

\begin{tabular}{lcr}
\hline \hline Object & $f_{\text {esc }}(\text { IPM })^{\mathrm{a}}$ & $f_{\text {esc }}(\mathrm{V} 08)^{\mathrm{b}}$ \\
\hline DEM L 10B ${ }^{\mathrm{c}}$ & 0.38 & -0.05 \\
DEM L 13 & 0.30 & 0.38 \\
DEM L 31 & 0.60 & 0.49 \\
DEM L 34 & 0.30 & 0.55 \\
DEM L 68 & 0.59 & 0.58 \\
DEM L 106 & 0.30 & 0.37 \\
DEM L 152+156 & 0.56 & 0.27 \\
DEM L 196 & 0.03 & 0.13 \\
DEM L 226 & 0.00 & -0.05 \\
DEM L 243 & 0.00 & 0.47 \\
DEM L 293 & 0.30 & -1.52 \\
DEM L 301 & 0.30 & 0.21 \\
DEM L 323+326 & 0.30 & 0.43 \\
\hline
\end{tabular}

Notes.

a $f_{\text {esc }}$ derived via ionization-parameter mapping.

b $f_{\text {esc }}$ derived from predicted $\mathrm{H} \alpha$ fluxes from Voges et al. (2008) and Equation (3).

${ }^{c}$ Composite objects whose $f_{\text {esc }}$ values reflect the luminosity-weighted contributions of individual optically thin and thick subregions.

a value of $f_{\text {esc }}$ that is half that for the optically thin objects, namely, $f_{\text {esc }}=0.3$. Since we have observations in only two diagnostic ions, some of the class 3 objects in reality may be quite optically thin (Section 2.2), and so we compare these estimates to direct measurements of optical depths using data from Voges et al. (2008), who compared the observed $L$ to predicted values based on the spectral types of individual ionizing stars for a sample of LMC H II regions. Although, as mentioned earlier, there is considerable uncertainty in optical depth estimates based on this method, it remains the most direct, quantitative way to check our results based on IPM.

Voges et al. (2008) adopted ionizing fluxes from the WM-basic models of Smith et al. (2002). These SEDs are intermediate in hardness among the different available modern codes, and they best fit the observed nebular emission-line spectra (Zastrow et al. 2011a). Due to the large uncertainty in determining spectral types of $\mathrm{O}$ stars from photometry alone, we restrict our comparison with the Voges et al. (2008) sample (their Tables 1 and 3 ) to objects having stellar spectral types determined at least in part by spectroscopic classifications, with a further requirement that at least half of the derived ionizing luminosity is attributed to stars with spectroscopic spectral types. These include a reanalysis of $\mathrm{H}$ II regions from Oey \& Kennicutt (1997), which is based entirely on spectroscopic classifications with individually measured reddenings, listed in Table 1 of Voges et al. We exclude DEM L 7, L 9, and L 55 because they are class 0 objects, and DEM L 229, which displays evidence of shock excitation.

Using the predicted $\mathrm{H} \alpha$ luminosities from Voges et al. (2008), and our new observed $\mathrm{H} \alpha$ luminosities, we calculate individual $f_{\text {esc }}$ values for each nebula according to

$$
f_{\text {esc }}=1-L_{\text {obs }} / L_{\text {predicted }}
$$

The predicted luminosity is derived from the expected rate of ionizing photons $Q\left(\mathrm{H}^{0}\right)$ assuming each absorbed LyC photon will result in $2.2 \mathrm{H} \alpha$ photons. In Table 2, we present the $f_{\text {esc }}$ values for the $13 \mathrm{H}$ II regions, comparing the rough estimates obtained from IPM as described above with measurements based on the data of Voges et al. (2008). Column 1 gives the DEM identifier for each object, and Column 2 gives our crude $f_{\text {esc }}$ 


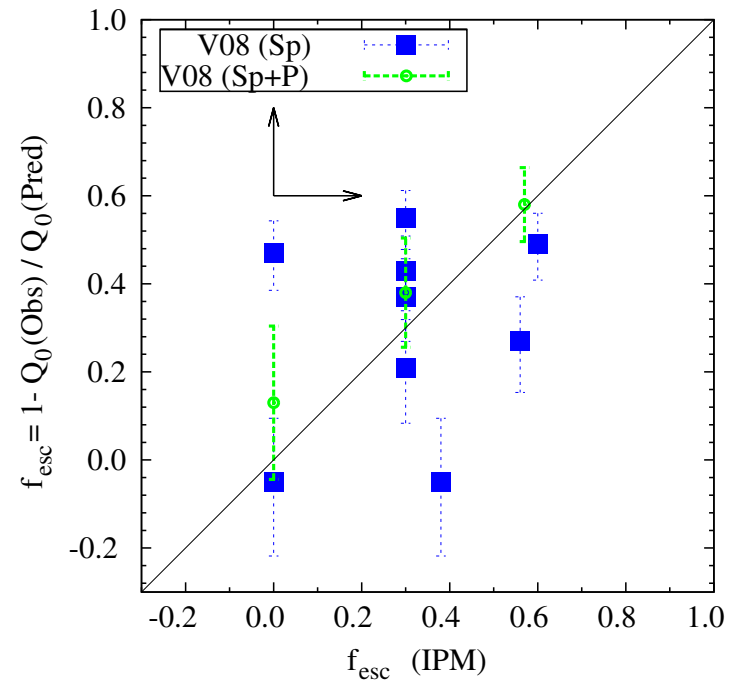

Figure 11. Our $f_{\text {esc }}$ values estimated from ionization-parameter mapping plotted against $f_{\text {esc }}$ calculated from observed and predicted ionization rates from Voges et al. (2008). The values of $f_{\text {esc }}$ derived from both methods are lower limits, illustrated by the orthogonal set of arrows.

(A color version of this figure is available in the online journal.)

estimated from IPM, as described above. Column 3 gives $f_{\text {esc }}$ estimates based on data from Voges et al. (2008) with values derived from known stellar spectral types.

Figure 11 plots the comparison between $f_{\text {esc }}$ estimated from our classification of optical depth based on IPM and the measured values based on the data of Voges et al. (2008). There is a general agreement between our crude estimates for $f_{\text {esc }}$ based on IPM and the measured values based on the observed ionizing stars, for all but one object; the standard deviation from the identity relation is $\sigma=0.23$, excluding DEM L 293 (see below). Although, as discussed in Section 2.2, our values for $f_{\text {esc }}$ are all lower limits, especially for objects categorized as optically thick, and the $f_{\text {esc }}$ values derived using Voges et al. (2008) are also lower limits, the surprisingly good correspondence suggests that both methods actually yield reasonable estimates of the optical depth.

The Voges et al. escape fraction of DEM L 293 is - 1.52, which is an unphysical value, placing it far beyond the bounds of the plot in Figure 11. The predicted ionizing luminosity in DEM L 293 is observationally attributed to only a single O3 III star. However, given the typical cluster mass in which O3 III stars form, additional, obscured, or overlooked ionization sources in this cluster are likely to be present. In particular, Walborn et al. (2002) identified an odd semi-stellar source within DEM L 293 that is brighter than the single O3 III star. If the ionizing luminosity of this source is equal to an O3 III star then the V08 $f_{\text {esc }}$ would then be -0.52 , much closer to the $f_{\text {esc }}$ value derived from IPM.

Overall, however, our extremely crude estimates of $f_{\text {esc }}$ based on IPM show surprisingly good agreement with the empirically measured $f_{\text {esc }}$. In spite of the fact that our estimates tend to yield lower limits, the general agreement confirms that objects appearing to be optically thick indeed tend to be radiation bounded. As mentioned in Section 2.1, this is also supported by their morphologies, which generally resemble smooth, Strömgren spheres. Furthermore, the occurrence of optically thick objects that appear to be density bounded is extremely rare, since this only happens occasionally for the very hottest spectral types (e.g., Figure 2). Figure 11 thus

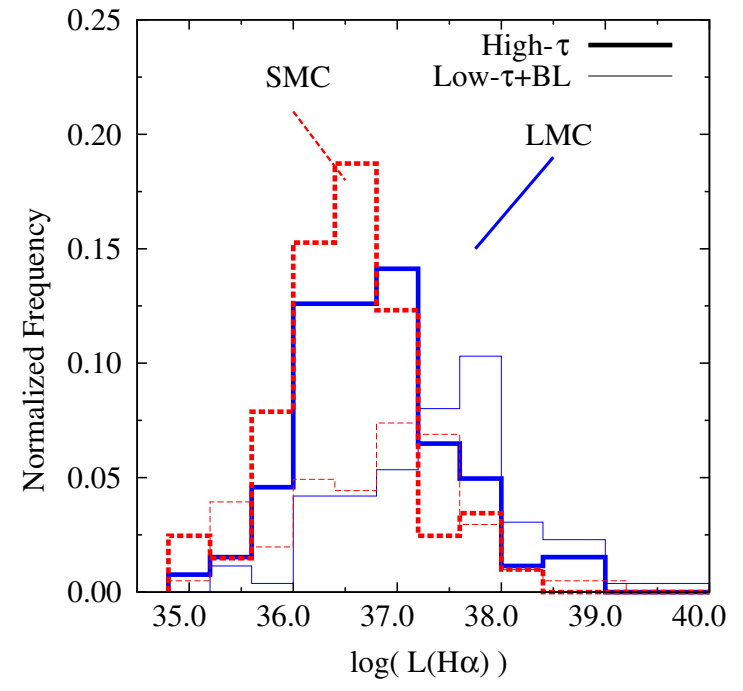

Figure 12. LFs for optically thin nebulae, including blister objects, and optically thick nebulae, shown with thin and thick lines, respectively. The LMC distributions are shown with solid lines (blue) and the SMC distributions are shown with dashed lines (red).

(A color version of this figure is available in the online journal.)

demonstrates the general viability of IPM as a diagnostic of nebular optical depth.

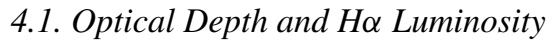

Table 3 summarizes the median nebular properties for each optical depth class as cataloged in Tables 5 and 6. Column 2 gives the number of objects in each class in the LMC. Column 3 gives the corresponding percentage of the total number of objects that are clearly photoionized, thus excluding class 0 and class 4 objects from the total numbers of photoionized nebulae. Columns 4 and 5 give the median $L$ and median $N(\mathrm{HI}$ ) (see Section 4.2 below) associated with the objects, respectively. Columns 6-9 list the same quantities for the SMC.

Figure 12 shows the $L$ distributions for the LMC (solid blue line) and SMC (dotted red line) of optically thin nebulae (thin lines) and optically thick nebulae (thick lines). The optically thin data include blister $\mathrm{H}$ II regions as defined above. The distributions are normalized by the number of $\mathrm{H}$ II regions in the last row of Table 3. Figure 12 and Table 3 show that for both galaxies, the $L$ distributions for optically thick objects peak at lower luminosities than those for the optically thin ones. This difference is larger in the LMC, producing a bimodal distribution, with the median $L$ for optically thin nebulae five times brighter than for the optically thick ones (Table 3). The median $L$ of optically thin SMC nebulae is only twice that of optically thick ones, as expected given the lower starformation rate in that galaxy and fewer luminous H II regions. However, the $L$ distributions for the different classes are similar between the two galaxies, showing peaks near similar values and similar ranges in luminosity. The role of dust in these trends is unclear. It is possible that two $\mathrm{H}$ II regions with similar ionizing luminosities will have different $f_{\text {esc }}$ if one has more dust than the other. This could explain the coexistence of optically thin and thick regions in the same luminosity bin.

In Figure 13, we plot the frequencies of optically thin nebulae as a function of $L$. Both galaxies exhibit a clear increase in the frequency of optically thin nebulae with increasing $\mathrm{H} \alpha$ luminosity. However, we stress that both optically thick and thin objects are found at almost all luminosities having $\log L<39.0$. 
Table 3

Median H II Region Properties

\begin{tabular}{|c|c|c|c|c|c|c|c|c|}
\hline \multirow[b]{2}{*}{ Class } & \multicolumn{4}{|c|}{ LMC } & \multicolumn{4}{|c|}{ SMC } \\
\hline & No. & $\%^{\mathrm{a}}$ & $\begin{array}{c}L \\
10^{36}\left(\mathrm{erg} \mathrm{s}^{-1}\right)\end{array}$ & $\begin{array}{c}N(\mathrm{H} \mathrm{I}) \\
10^{21}\left(\mathrm{~cm}^{-2}\right)\end{array}$ & No. & $\%^{\mathrm{a}}$ & $\begin{array}{c}L \\
10^{36}\left(\mathrm{erg} \mathrm{s}^{-1}\right)\end{array}$ & $\begin{array}{c}N(\mathrm{H} \mathrm{I}) \\
10^{21}\left(\mathrm{~cm}^{-2}\right)\end{array}$ \\
\hline 0 -Indeterminate & 130 & $\ldots$ & 3.7 & 1.8 & 7 & $\ldots$ & 0.05 & 6.5 \\
\hline 1-Opt Thick & 158 & 60 & 3.6 & 2.8 & 132 & 62 & 2.1 & 6.4 \\
\hline 2-Blister & 58 & 18 & 18.8 & 1.9 & 41 & 19 & 4.7 & 5.1 \\
\hline 3-Opt Thin & 46 & 22 & 19.7 & 2.0 & 30 & 14 & 4.0 & 6.2 \\
\hline 4-Shocked & 9 & $\ldots$ & 15.6 & 1.8 & 4 & 2 & 1.5 & 5.7 \\
\hline$(2)+(3)$ & 104 & 40 & 19.2 & 1.9 & 71 & 33 & 4.5 & 5.3 \\
\hline$(1)+(2)+(3)$ & 262 & 100 & 5.5 & 2.5 & 203 & 100 & 2.6 & 5.9 \\
\hline
\end{tabular}

Note. ${ }^{\text {a }}$ Percentages are calculated for photoionized objects, based on the total values in the bottom row.

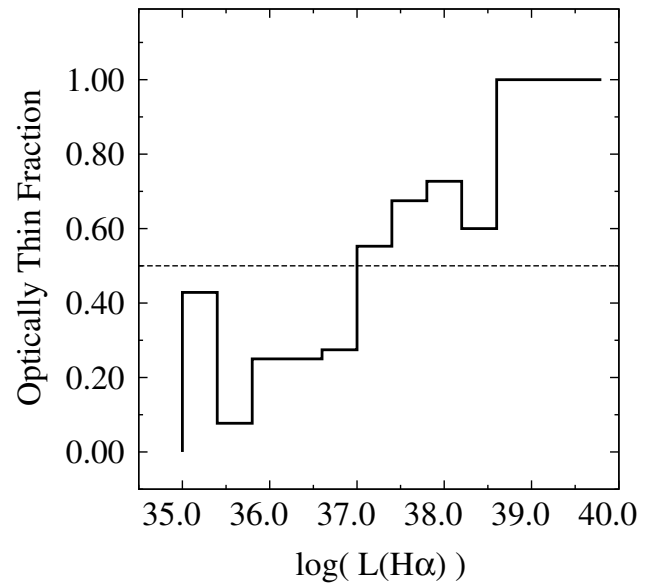

(a) LMC

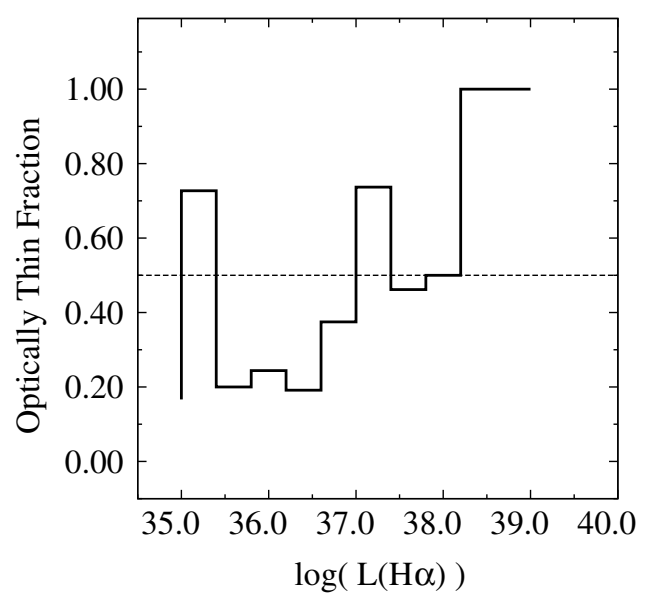

(b) SMC

Figure 13. Fraction of optically thin $\mathrm{H}$ II regions as a function of $L$ is shown for the LMC and SMC in panels (a) and (b), respectively. Optically thin objects dominate above a fraction of 0.5 , indicated by the horizontal dotted line. The lowest $L$ bins likely include contamination by planetary nebulae.

Table 3 also shows that the frequencies of optically thin objects, including blisters, are similar between the two galaxies, $40 \%$ and $33 \%$ in the LMC and SMC, respectively. Furthermore, there is a transition luminosity above which optically thin nebulae dominate, $\log L=37.0$, occurring at the same luminosity in both galaxies. Beckman et al. (2000) speculated that such a transition is responsible for possible discontinuities observed near $\log L=38.6$ in extragalactic H II LFs, but our data clearly show that optically thin objects dominate at luminosities a full 1.6 dex lower in $L$.
It will be interesting to see how strongly our transition value of $\log L=37.0$ depends on galaxy properties. This relatively low luminosity corresponds to nebulae ionized stochastically by single $\mathrm{O}$ stars or substantially evolved associations and clusters (Oey \& Clarke 1998). Thus, most of the objects typically apparent in Figures 6 and 7, as well as those typically detected in local surveys (e.g., Thilker et al. 2002), are the more luminous $\mathrm{H}$ II regions, which are mostly, but not all, optically thin. The most luminous objects have the highest likelihood of being optically thin, including 30 Doradus in the LMC and the N66 in the SMC. These are indeed found to be optically thin in our study, a result consistent with the findings of Pellegrini et al. (2011) in 30 Dor, and the low optical depths found for other giant extragalactic H II regions (e.g., Castellanos et al. 2002).

\subsection{Relation with the Neutral ISM}

The neutral ISM represents the default environment into which ionizing photons from optically thin regions are deposited, and its properties are fundamental to the radiative transfer of the LyC. The Magellanic Clouds were mapped in H I with the Australia Telescope Compact Array by Kim et al. (2003; LMC) and Stanimirović et al. (1999; SMC). The LMC H I data have a 60 arcsec resolution over the $11.1 \times 12.4$ survey area; the SMC H I data have a resolution of 98 arcsec over the $5^{\circ} \times 5^{\circ}$ field. The LMC is a face-on disk galaxy, while the SMC has a more amorphous, three-dimensional (3D) irregular morphology. We now explore the relationship between nebular optical depth and the neutral ISM.

Figure 14 traces the propagation of radiation in the LMC (panel (a)) and SMC (panel (b)) using [S II]/[O III] (red), $[\mathrm{O}$ III] $/ \mathrm{H} \alpha$ (blue), and $N(\mathrm{HI})$ (green). The contrast in ionization morphology between the two galaxies is striking. As ionizing radiation enters the diffuse ISM, it encounters a combination of ionized and neutral gas. The LMC neutral disk has been disrupted, forming shells and filaments surrounding the ionized gas (Kim et al. 1998). These structures are believed to be the result of stellar feedback acting on the ISM (e.g., Oey \& Clarke 1997). Often, optically thin H II regions line the edges of large $\mathrm{H}$ I shells, radiating LyC photons into their interiors. A few examples are highlighted with arrows in the LMC (Figure 14). These large-scale H I structures appear to allow ionizing radiation to travel hundreds of parsecs without being absorbed.

The SMC, on the other hand, has a less fragmented $\mathrm{HI}$ structure (Stanimirović et al. 1999). The neutral ISM in this galaxy is much more diffuse and less filamentary than that in the LMC (Oey 2007). Intense and vigorous star formation 


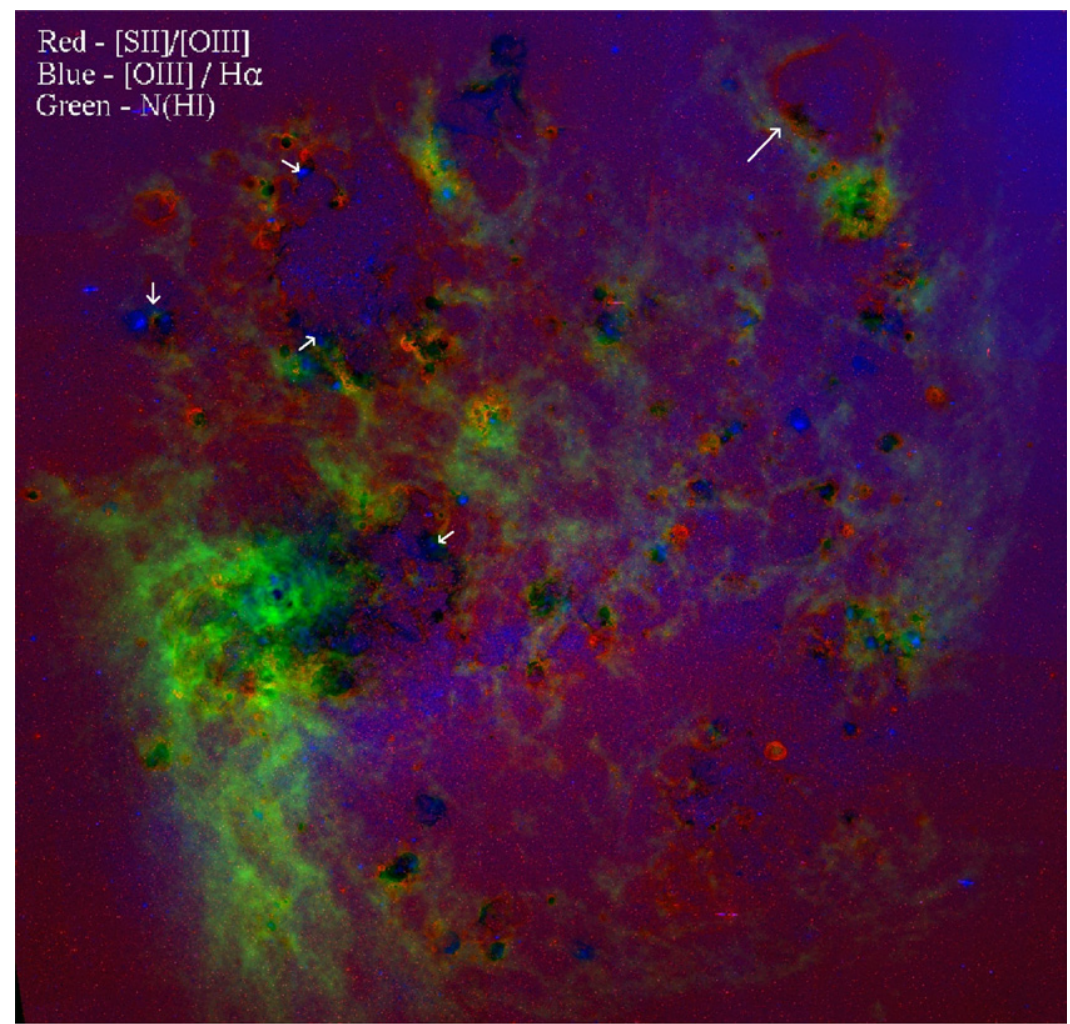

(a) LMC

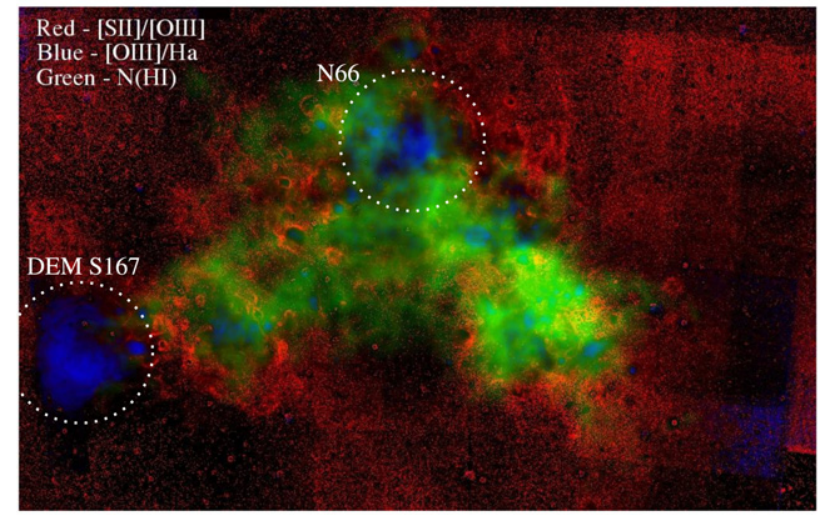

(b) SMC

Figure 14. Composite images of the LMC (a) and SMC (b), which contrast the different ISM structure seen in the two galaxies. [O III]/H $\alpha$ (blue), [S II]/[O III] (red), and $N(\mathrm{HI})$ (green) respectively trace high-excitation nebulae, low-excitation gas, and neutral gas along the line of sight.

(A color version of this figure is available in the online journal.)

is at the center of the two most prominent Hi masses. The first is coincident with N66 near the northern boundary of the galaxy. Lines of sight toward this optically thin region show that $N(\mathrm{HI})$ anti-correlates with highly ionized gas (Figure 14). Thus, in this region, $\mathrm{H}_{\mathrm{I}}$ is being disrupted by the ionizing radiation entering the diffuse ISM. The second region is located in the SW portion of the galaxy. Despite a high $N(\mathrm{HI})$, this region contains many optically thin nebulae, which form a large complex filled with a highly ionized DIG. To improve our sensitivity to ionization transitions in the DIG, we applied an $11 \times 11 \mathrm{arcsec}^{2}$ median filter to the $\left[\mathrm{S} \mathrm{II}_{\mathrm{II}}\right]$ and $[\mathrm{O} \mathrm{III}]$ data, creating a smoothed [S II]/[O III] map. The region is seen in Figure 15 in the inverted map (left) and $N(\mathrm{HI})$ (right). The enhanced sensitivity reveals an ionization transition zone coincident with the edge of the $\mathrm{H}$ I distribution. Thus, the $\mathrm{H}_{\mathrm{I}}$ gas appears to be trapping the ionizing radiation, while individual nebular $f_{\text {esc }}$ depends on the detailed morphology.
Given the strong morphological contrast between the two galaxies, in the H I properties and star formation intensity, the quantitative similarities in the nebular optical depths found above in Section 4 are surprising. In particular, despite expectations that the reduced $N(\mathrm{HI})$ and higher star formation intensity of the LMC would lead to more optically thin nebulae, we saw above that the relative frequency of optically thin and thick objects are similar between the two galaxies (Table 3). It will be important to see whether other galaxies also yield similar relative frequencies.

\subsection{Optical Depth and $N(\mathrm{HI})$}

Figure 16 shows the $N(\mathrm{HI})$ distribution for optically thin (thin lines) and thick nebulae (thick lines) for the LMC (solid blue lines) and SMC (dotted red lines). We used the $N(\mathrm{HI})$ maps of Kim et al. (2003) for the LMC and Stanimirović et al. (1999) for the SMC, to average the $N(\mathrm{HI})$ within the individual H II 

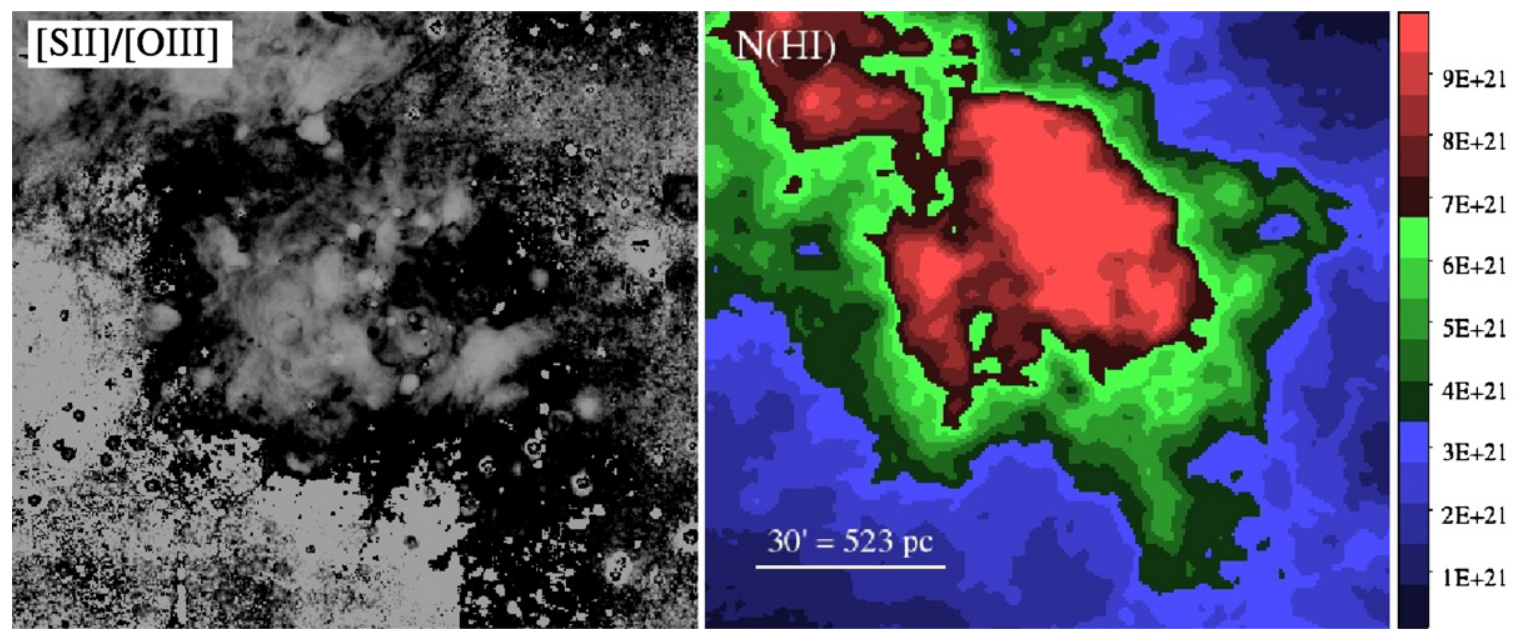

Figure 15. Southwest region of the SMC in $\left[\mathrm{S} \mathrm{III} /\left[\mathrm{O}\right.\right.$ III] (left), smoothed with an $11 \times 11 \operatorname{arcsec}^{2}$ median filter. We have inverted the gray scale to better highlight low-ionization regions, thus black corresponds to areas of low ionization. Surrounding the entire complex is an ionization transition zone (black). This transition zone is coincident with the boundaries of a massive H I cloud seen in the N(H I) map of Stanimirović et al. (1999; right).

(A color version of this figure is available in the online journal.)

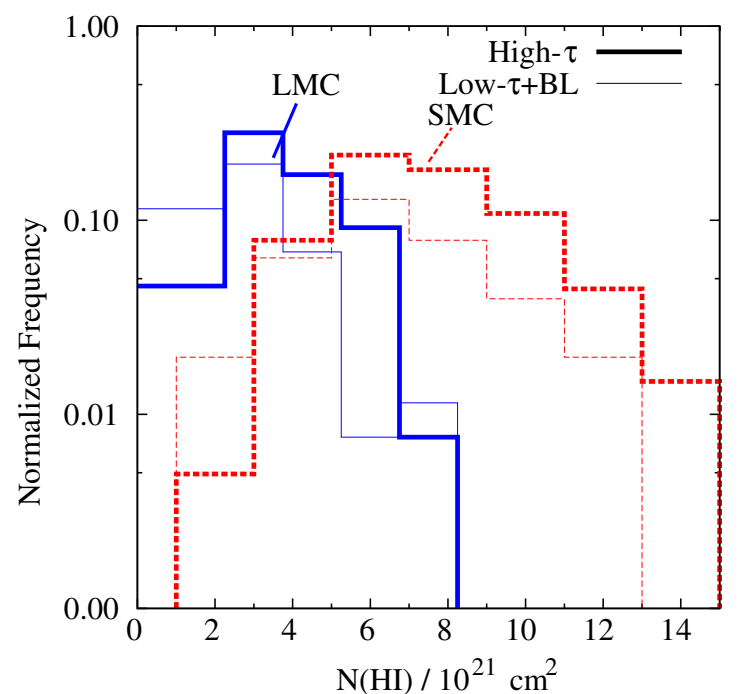

Figure 16. Normalized $N(\mathrm{HI})$ distributions for optically thin and optically thick nebulae, shown with thin and thick lines, respectively. The LMC and SMC distributions are shown with solid lines (blue) and dashed lines (red), respectively. These have been scaled by the total number of objects in each group.

(A color version of this figure is available in the online journal.)

region apertures for each object, as defined in Section 3.1. We caution that these measurements correspond to the $N(\mathrm{HI})$ along the line of sight toward the objects. Because the SMC has a more 3D geometry than the LMC, which is an almost face-on disk, the $N(\mathrm{HI})$ measurements for the SMC include a larger contribution from foreground and background ISM than in the LMC. However, we note that the SMC metallicity and dust content are only one-fifth that of the LMC; thus, for the same $N(\mathrm{H} \mathrm{I}), \tau_{\text {LyC }}$ will be lower in the SMC. Hence the less abundant dust may somewhat offset the effect of increased $N(\mathrm{HI})$ in this galaxy. Still, because of the contrasting galaxy morphologies, the difference in median $N(\mathrm{H}$ I $)$ between optically thin and thick populations within each galaxy is smaller than the difference in global median $N(\mathrm{HI})$ between the two galaxies. Specifically, the median value of all LMC HiI regions is 2.4 times lower than in the SMC (Table 3), while the ratio of the median $N(\mathrm{HI})$ for optically thick (class 1 ) to thin (class $2+3$ ) objects is 1.5 and 1.2 in the LMC and SMC, respectively. Figure 16 shows that, for both galaxies, the $N(\mathrm{HI})$ distributions for optically thin and thick nebulae are similar to each other, but that the former are weighted more toward lower columns, as expected. However, we note that even in the LMC, which has minimal lineof-sight projection effects, the $N(\mathrm{HI})$ distribution for optically thin objects extends up to $N(\mathrm{HI})=8 \times 10^{21} \mathrm{~cm}^{-2}$, a value as high as that for the optically thick ones.

In Figure 17, we show the relative frequency of optically thin nebulae as a function of $N(\mathrm{HI})$ in the LMC and SMC. As expected, we see a strong decrease in the frequency of optically thin objects with increasing $\mathrm{H}$ I column, although as emphasized above, there are still optically thin objects found near the highest $N(\mathrm{H} \mathrm{I})$. In both galaxies, there is a transition $N(\mathrm{HI})$ below which optically thin nebulae constitute the majority, at $3 \times 10^{21} \mathrm{~cm}^{-2}$ in the LMC and $6 \times 10^{21} \mathrm{~cm}^{-2}$ in the SMC. The SMC transition $N(\mathrm{HI})$ is two times higher than in the LMC, which is consistent with the 2.4 times higher median $N(\mathrm{H} \mathrm{I})$ value for all $\mathrm{H}$ II regions in the LMC relative to the SMC, due to line-of-sight ISM projection. Thus, in spite of the very different H I morphology between the two galaxies, the quantitative relationship between nebular optical depth and H I column is remarkably similar.

\section{GLOBAL ESCAPE FRACTIONS}

While the frequency of optically thin versus thick $\mathrm{H}$ II regions is similar in both galaxies, it is the structure of the diffuse ISM that ultimately determines how many ionizing photons heat the galaxy, and how many escape into the IGM, a quantity crucial to our understanding of cosmic evolution. Figure 14 highlights how, in comparison to the SMC, the evacuated ISM of the LMC allows the radiation produced in these regions to travel farther, and perhaps leave the galaxy. In the SMC, much of the ionizing radiation escaping $\mathrm{H}$ II regions is unable to penetrate the higher apparent $\mathrm{H}$ i column. We now explore the global escape fractions for the Magellanic Clouds.

\subsection{H II Region Location}

Gnedin et al. (2008) highlighted the importance of $\mathrm{H}$ II region location on the escape of ionizing radiation from galaxies. 


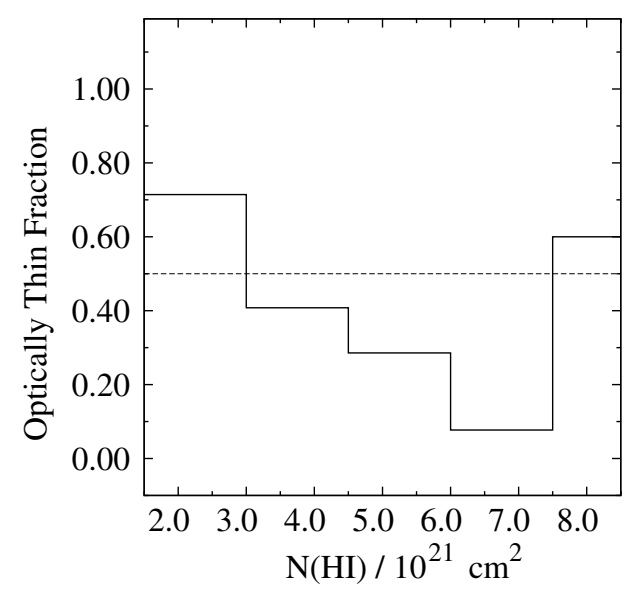

(a) LMC

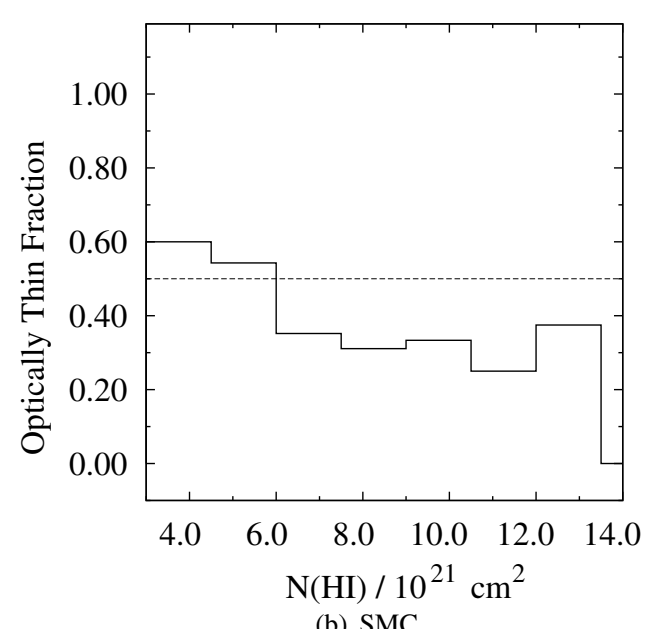

Figure 17. Frequency of optically thin $\mathrm{H}_{\mathrm{II}}$ regions vs. line-of-sight $N(\mathrm{HI})$ within the object apertures, for the LMC and SMC in panels (a) and (b), respectively. Optically thin objects dominate above a fraction of 0.5 , indicated by the horizontal dotted line.

In the LMC and SMC, the largest and most luminous H II regions are found toward the galaxy edges, and these objects are apparently optically thin. Take for example DEM S167, seen in the southeast extreme of the SMC (Figures 7 and 14(b)). In Figure 18, the transition in ionization is shown in [S II]/[O III] (left) coincident with the Hi shell SSH97 499 (Stanimirović et al. 1999; right). These diagnostics indicate that part of the region is optically thick. However, there is [O III] emission extending to the south, well beyond the ionization transition zone. The existence of extended [O III] implies a large nebular escape fraction. The significance of escaping radiation from DEM S167 is amplified by its location near the edge of the SMC (Figure 14(b)).

Following Voges et al. (2008), we compare the expected $\mathrm{H} \alpha$ luminosity from the stellar population to the observed value. The predicted $\mathrm{H} \alpha$ luminosity from seven known $\mathrm{O}$ stars, ranging from $\mathrm{O} 4 \mathrm{~V}$ to $09.5 \mathrm{~V}$, was derived using the observed relation between spectral type and $Q\left(\mathrm{H}^{0}\right)$ from Martins et al. (2005). This includes a rare, well-studied $\mathrm{WO} 4+\mathrm{O} 4$ binary for which we adopt the luminosities reported by St-Louis et al. (2005) equal to $75 \%$ of the total ionizing budget. The predicted $\mathrm{H} \alpha$ luminosity is $\log L=38.137$, implying $f_{\text {esc }}=27 \%$, consistent with our estimate of $30 \%$ from IPM. As the easternmost known SMC $\mathrm{H}$ II region, its blister opens away from the galaxy, making it a prime candidate to contribute to the galactic escape fraction. The escaping UV radiation would be detectable only from certain directions as predicted by Gnedin et al. (2008).

\subsection{H II Region Luminosity}

The most luminous HII region in the LMC is 30 Doradus, ionized by the cluster R136a. It has a reddened luminosity of $\log L=39.66$, and it is ionized by hundreds of $\mathrm{O}$ stars. Similarly, at $\log L=38.82$, the brightest nebula in the SMC is N66, ionized by at least $30 \mathrm{O}$ stars in the cluster NGC 346. We can see in Figures 6 and 7 that these luminous objects are strongly optically thin, based on their very extended [O III] emission. Furthermore, they are not deeply embedded in their respective galaxies, implying that these massive regions produce ionizing radiation that may escape into the IGM.

To examine the LyC photon path lengths from these two, luminous objects, we show the azimuthally averaged [S II]/[O III] ratio for these regions in Figure 19, centered on the main ionizing clusters R136a (top) and NGC 346 (bottom). Small, discrete $\mathrm{H}$ II regions that are projected in the line of sight within these regions are excluded from the azimuthal averages in Figure 19. Figure 19 also shows the average radial $N(\mathrm{HI})$. The profile of 30 Doradus is plotted to maximum radii where the gradient of both quantities is equal to zero and marks the distance at which the ionizing radiation from these sources is no longer dominant. N66 is fainter, so it is less clear exactly where the influence of its ionizing source ends. Clearly, the extended [O III] emission from the gas surrounding both objects requires a photoionizing source of high-energy photons from R136a and NGC 346, which dominate the ionization of the DIG out to at least $600 \mathrm{pc}$, whether or not this gas was ever associated with the HII region, or is just part of the DIG. This is in agreement with the enhanced $\mathrm{H} \alpha$ surface brightness of 30 Dor out to $850 \mathrm{pc}$ noted by Kennicutt et al. (1995).

Figure 19 shows that the peak $N(\mathrm{H}$ I) associated with optically thin objects can occur at radial distances that are well within the radial limits of the photoionized region. This suggests that the neutral and molecular ISM is highly inhomogeneous and clumpy, with large holes or clear areas that allow the escape of ionizing radiation. This situation is similar to the ionization cone detected in NGC 5253 (Zastrow et al. 2011b). In particular, the radial bins used to produce Figure 19 mask important features in the $[\mathrm{S} \mathrm{II}] /[\mathrm{O} \mathrm{III}]$ and $N(\mathrm{HI})$ distributions around 30 Doradus. These include narrow, radial projections containing continuous regions of highly ionized gas extending $1 \mathrm{kpc}$ in various directions from 30 Doradus. There is no evidence for additional ionizing sources that can explain the extended ionization, so we argue that the variation in path length is due to variations in ISM density. East of the ionizing source, R136a, we see a complex of edge-on filaments (Figure 20), associated with the giant H I shell LMC 2 (Meaburn 1980). These filaments form a continuous arc over $500 \mathrm{pc}$ in length, at a distance ranging from 0.6 to $0.8 \mathrm{kpc}$ from R136a, with strong [O III] facing the ionizing cluster, and strong [S II] facing away from it, as shown by the arrows in the figure. This ionic stratification confirms that the ionizing photons striking these filaments or sheets originate from R136a. Similar filaments are detected west of N66 (Figure 14(b)), opposite the bulk of the SMC. Unfortunately, our data do not extend far enough to look for filaments beyond DEM S167 in the direction away from the SMC. However, we see that both the location and luminosity of these giant $\mathrm{H}$ II regions strongly influence the likelihood of LyC radiation escaping from the host galaxies. 

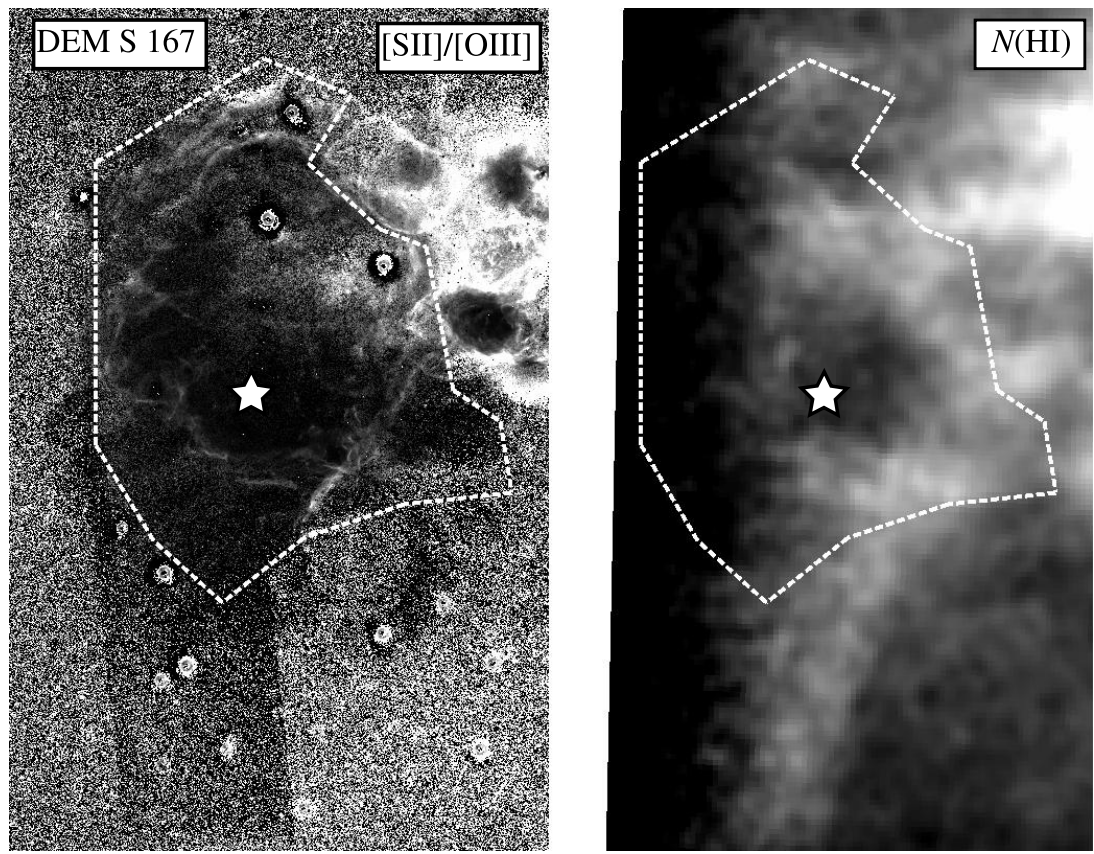

Figure 18. Optically thin H II region DEM S 167 located at the southeast boundary of the SMC. The left panel, showing [S II]/[O III], shows a strip running north to south with spuriously enhanced levels of [O III]. However, extended [O III] emission (black) is clearly detected beyond the southern nebular boundary, in excess of the artifact's signal. The right panel shows the H I column density map from (Stanimirović et al. 1999), demonstrating that the [O III] emission extends beyond the H I shell. The star indicates the location of the WO4+O4 binary, and the ionization-based boundary of the nebula is shown as a dashed line.

\subsection{Integrated H II Region Escape Fractions}

To understand the LyC radiation transfer within galaxies, it is of central interest to evaluate the luminosity-weighted, mean LyC escape fraction $\left\langle f_{\text {esc }}\right\rangle$ of all the nebulae within each galaxy. We first calculate the total $\mathrm{H}$ II region "escape luminosity" $L_{\text {esc }}$ in terms of individual, observed $\mathrm{H}$ II region luminosities using

$$
L_{\mathrm{esc}}=\sum_{i}\left(L_{i} \times \frac{f_{\mathrm{esc}, i}}{1-f_{\mathrm{esc}, i}}\right),
$$

where $i$ represents the $i$ th object in the given galaxy. Note that $L$ is the observed luminosity, as before, which is related to the total ionizing luminosity $L_{\text {tot }}$ by $L=L_{\text {tot }}\left(1-f_{\text {esc }}\right)$. We again adopt $f_{\text {esc }}=0.6$ for optically thin nebulae and 0.3 for blister regions. Optically thick nebulae contribute no escaping radiation, but add to the total observed $\mathrm{H} \alpha$ luminosity. The total escape luminosities for the individual object classes are listed in Table 4. The total $L_{\text {esc }}$ from all $\mathrm{H}$ II regions in the galaxies are $\log L_{\mathrm{esc}}=40.1$ in the LMC and $\log L_{\mathrm{esc}}=39.2$ in the SMC.

Next, we calculate the luminosity-weighted $\mathrm{H}$ II region escape fraction in each galaxy according to

$$
\left\langle f_{\mathrm{esc}}\right\rangle=\frac{\sum_{i} L_{\mathrm{esc}, i}}{\sum_{i}\left(L_{\mathrm{esc}, i}+L_{i}\right)} .
$$

We find the lower limit on $\left\langle f_{\text {esc }}\right\rangle$ in the LMC and SMC to be $0.42-0.51$ and 0.40 , respectively. The lower LMC value corresponds to a scenario where indeterminate, class 0 objects are optically thick, while the upper limit assumes that they are optically thin. In the LMC these objects account for $<14 \%$ of the total $\mathrm{H}$ II region luminosity, while they do not make any significant contribution in the SMC. We have not included the uncertainty due to photometry, which is $20 \%$ for individual objects and introduces an error of $22 \%$ to our $f_{\text {esc }}$ calculations. Therefore, our final lower limits on $\left\langle f_{\text {esc }}\right\rangle$ in the LMC and SMC are $0.42 \pm 0.09$ and $0.40 \pm 0.09$, respectively.

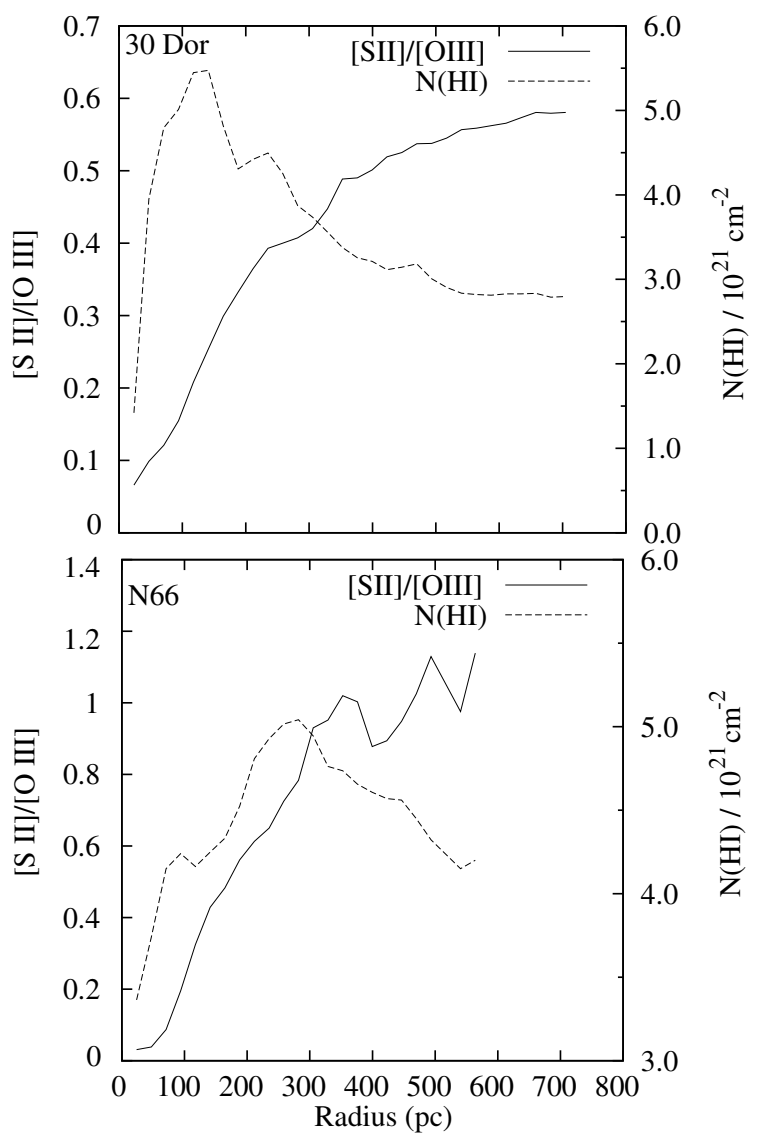

Figure 19. Large-scale radial profiles of $\left[\mathrm{S}_{\mathrm{II}}\right] /[\mathrm{O} \mathrm{III}]$ and $N(\mathrm{HI})$, averaged in annuli centered on R136a in 30 Dor (top) and NGC 346 in N66 (bottom). Nearby $\mathrm{H}$ II regions falling within the annuli were masked. 


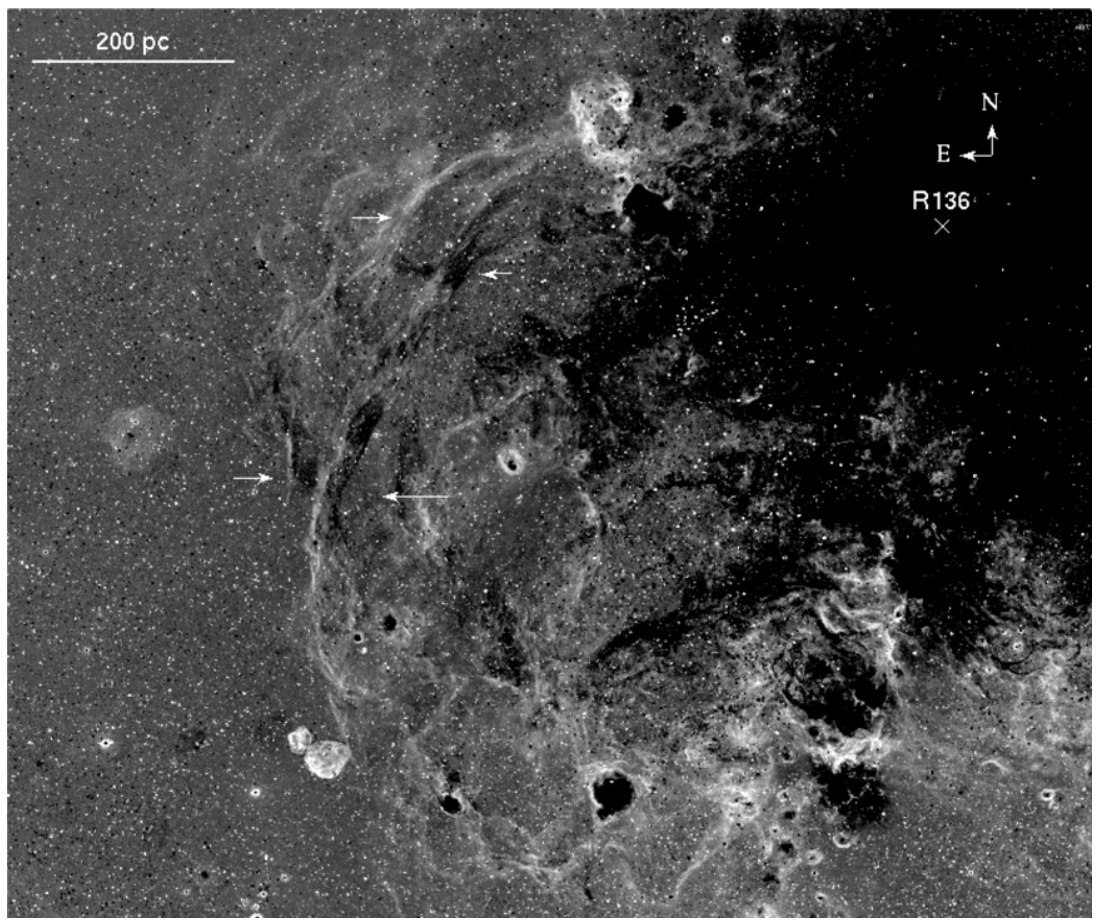

Figure 20. Complex region east of R136a seen in [S II]/[O III]. R136a is marked in the upper right corner of the image. Marked by arrows are ionized filaments which exhibit ionization stratification over nearly $500 \mathrm{pc}$ in length. The orientation of the stratification is evidence of a centrally located illuminating source at a location consistent with 30 Doradus.

Table 4

Global Radiative Transfer Properties of Nebular Populations

\begin{tabular}{lccccccc}
\hline \hline & \multicolumn{3}{c}{ LMC } & & \multicolumn{3}{c}{ SMC } \\
\cline { 2 - 4 } \cline { 7 - 8 } & $\log L^{\mathrm{a}}$ & $\log L_{\mathrm{esc}}^{\mathrm{b}}$ & $\left\langle f_{\mathrm{esc}}\right\rangle^{\mathrm{b}}$ & & $\log L^{\mathrm{a}}$ & $\log L_{\mathrm{esc}}^{\mathrm{b}}$ & $\left\langle f_{\mathrm{esc}}\right\rangle^{\mathrm{b}}$ \\
\hline Indeterminate (class 0) & 39.3 & $\ldots$ & $\ldots$ & & 36.6 & $\ldots$ & $\ldots$ \\
Opt Thick (class 1) & 39.4 & $\ldots$ & 0.0 & & 38.9 & $\ldots$ & 0.0 \\
Blister (class 2) & 39.5 & 39.1 & 0.3 & & 38.7 & 38.4 & 0.3 \\
Opt Thin (class 3) & 39.9 & 40.0 & 0.6 & & 39.1 & 39.2 & 0.6 \\
Classes 1 + 2 + 3 & 40.2 & 40.1 & 0.42 & & 39.4 & 39.2 & 0.40 \\
Kennicutt et al. (1995) & 40.2 & 40.0 & 0.35 & & 39.5 & 39.3 & 0.41 \\
\hline
\end{tabular}

Notes.

${ }^{\text {a }}$ Columns 2 and 5 give the sum of the observed $L$.

${ }^{\mathrm{b}} L_{\mathrm{esc}}$ and $\left\langle f_{\mathrm{esc}}\right\rangle$ are lower limits.

c $L_{\text {esc }}$ from Kennicutt et al. (1995) corresponds to their measured DIG luminosity, $L_{\text {DIG }}$.

Because we are using only two line ratios to constrain $f_{\text {esc }}$, we again note that these estimates for $\left\langle f_{\text {esc }}\right\rangle$ are lower limits, although as discussed above, they are not strong lower limits. We can compare our results to the estimated $\left\langle f_{\text {esc }}\right\rangle$ for the Magellanic Clouds by Kennicutt et al. (1995), who adopted the DIG luminosity for $L_{\text {esc }}$. They found $\left\langle f_{\text {esc }}\right\rangle=0.35$ and 0.41 in the LMC and SMC, respectively, which agree well with our estimates. Table 4 gives the total $L, L_{\mathrm{esc}}$, and $\left\langle f_{\mathrm{esc}}\right\rangle$ for the $\mathrm{H}$ II region populations listed in Column 1, with LMC and SMC values shown on the left and right side of the table, respectively.

The similarities in $\left\langle f_{\text {esc }}\right\rangle$ between the two galaxies are not due to H I distributions, which, as we saw above, differ strongly. Instead, they apparently result from the brightest optically thin nebulae. In the LMC, 30 Dor contributes nearly $60 \%$ of the total LMC escape luminosity. There is a similar situation in the SMC, where N66, ionized by the cluster NGC 346, contributes an estimated $50 \%$ of the total escape luminosity in that galaxy.

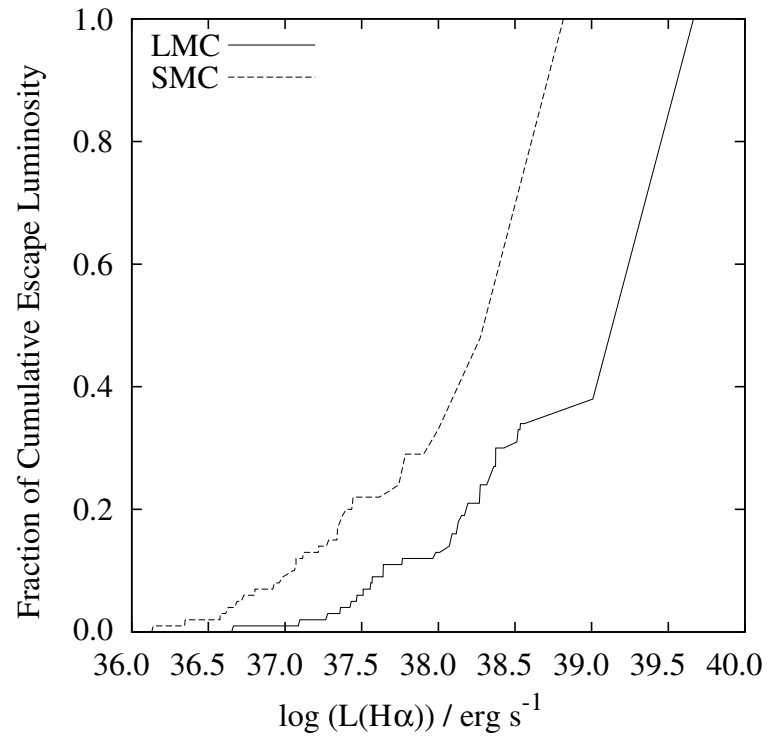

Figure 21. Cumulative fractional $L_{\mathrm{esc}}$ as a function of $\log L$ for the LMC (solid line) and SMC (dashed line).

Looking at Figure 21, the cumulative fractional $L_{\mathrm{esc}}$ as a function of observed $L$, we find that in the SMC only $30 \%$ of the escaping ionizing radiation comes from objects with $\log L<38$.0. In the LMC, the contribution is near $10 \%$ for the same range of $L$. Thus, the dominant contribution to the escape luminosity is from objects more luminous than $\log L>38.0$. Therefore, it is not surprising that the derived luminosity-weighted $\left\langle f_{\text {esc }}\right\rangle$ is similar between the two galaxies, when both (1) the total escaping luminosity is dominated by the bright objects and (2) a single $f_{\text {esc }}$ is assumed to describe all optically thin nebulae. 


\subsection{Ionizing the WIM}

Now, we confront an important question: Can ionizing radiation escaping from optically thin star-forming regions explain the luminosity of the WIM? Previous studies estimated that $\sim 40 \%$ of the WIM (or DIG) ionization is due to isolated field stars, and optically thin $\mathrm{H}$ II regions powered by clusters contribute the remaining $60 \%$ (e.g., Oey et al. 2004; Hoopes \& Walterbos 2000; Oey \& Kennicutt 1997; Miller \& Cox 1993). From Table 4, we find that $\left\langle f_{\text {esc }}\right\rangle$ and $L_{\text {esc }}$ from the $H$ II regions alone are enough to balance the observed LMC DIG recombination rate observed by Kennicutt et al. (1995), and that H II regions account for $84 \%$ of the DIG luminosity in the SMC. Including photometric errors, the DIG of both galaxies can be powered by optically thin radiation from $\mathrm{H}$ II regions alone. This is not at odds with the previous results when we consider that not all field stars are sitting naked in the DIG. Many are ionizing discrete nebulae, so that simply adding the ionizing luminosities of all field stars to $L_{\text {esc }}$ will overestimate its value.

We have a unique opportunity to examine this quantitatively in the SMC: the RIOTS4 survey of Oey \& Lamb (2011) is the only complete survey to target field massive stars in an external galaxy, and it includes 115 spectroscopically confirmed field O stars from the Oey et al. (2004) sample of field OB stars. Of these SMC field O stars, 60 show no associated nebular emission. Using $Q\left(\mathrm{H}^{0}\right)$ from Martins et al. (2005) to convert the stellar spectral types from Oey \& Lamb (2011), the total ionizing flux from these is equivalent to $12 \%$ of the DIG ionization rate. There are an additional 27 stars whose location inside nebulae is ambiguous. If we assume that their ionizing luminosity also streams into the DIG, field $\mathrm{O}$ stars produce a total ionization rate equivalent to $23 \%$ of the DIG. If we further assume that no ionizing radiation escapes the galaxy, then the DIG emission measured by Kennicutt et al. (1995) reflects the combined ionizing radiation from field stars and from optically thin $\mathrm{H}$ II regions, with $77 \%-88 \%$ of the ionizing radiation originating from $\mathrm{H}$ II regions. Using Equation (4), an SMC aggregate $\left\langle f_{\text {esc }}\right\rangle \sim 0.38-0.41$, instead of 0.40 (Table 4 ), would result in $\mathrm{H}$ II regions producing $77 \%-88 \%$ of the DIG luminosity in Table 4 , which is within our $f_{\text {esc }}$ uncertainty caused by photometry.

\subsection{Galactic Escape Fractions from the Magellanic Clouds}

With ample evidence of the great distances that ionizing radiation travels from massive star-forming regions, we can make an initial quantitative estimate of $f_{\text {esc,gal }}$, the galactic escape fraction, by comparing the aggregate escape luminosities from Equation (4) to the DIG luminosity $L_{\mathrm{DIG}}$, where

$$
f_{\text {esc }, \text { gal }}=\left(L_{\text {esc }}-L_{\text {DIG }}\right) / L_{\text {tot }} \text {. }
$$

The quantities needed to calculate $f_{\text {esc, gal }}$ are listed in Table 4, where $L_{\text {tot }}$ is the sum of observed $L$ and $L_{\mathrm{esc}}$, as before. We see that in the SMC, $f_{\text {esc,gal }}=0$. However, as discussed above, this neglects the contribution from a known population of massive field stars. As calculated in Section 5.4, the ionizing radiation from truly isolated stars in the SMC is $12 \%-23 \%$ of the DIG luminosity since about half of these field stars reside in $\mathrm{H}$ II regions. In the SMC, this yields a lower limit to $f_{\text {esc,gal }}$ of $4 \%-9 \%$. For the LMC, Table 4 shows that the ionizing luminosity escaping $\mathrm{H}$ II regions is also about the same as the value needed to explain the LMC DIG, without accounting for an unknown population of field $\mathrm{O}$ stars. It is reasonable to assume that the field star ionizing luminosity relative to $\mathrm{H}_{\text {II }}$ region $L_{\mathrm{tot}}$ is similar (Oey et al. 2004) in the LMC and SMC (0.05-0.11). From Table 4 and Equation (4) we find that a lower limit to $f_{\text {esc, gal }}$ in the LMC is $11 \%-17 \%$.

It is important to bear in mind that our constraints on nebular $\left\langle f_{\text {esc }}\right\rangle$ technically are lower limits, as stressed in Section 2 . The ranges in galactic escape fraction quoted above only reflect the uncertainties in the field star population. Thus, it is possible that $f_{\text {esc,gal }}$ may be underestimated in one or both of the galaxies; the crude estimates in Table 4 preclude any conclusive results. Future work is needed to quantitatively improve these constraints. Further efforts may be directed at obtaining more definitive IPM by adding imaging in more ions, or modeling diagnostic emission lines in filaments ionized by distant sources like those for 30 Doradus and N66, which can constrain the ionizing photon flux and SED of these dominant objects.

\section{CONCLUSIONS}

We have demonstrated the power of spatially resolved IPM to quantitatively probe the optical depth of $\mathrm{H}$ II regions to the LyC. Our CLOUDY photoionization simulations show that spatially resolved emission-line ratio mapping reveals the presence or absence of ionization stratification that diagnoses the optical depth of photoionized regions. The technique also constrains the optical depth in the line of sight. We show that IPM in only [S II] and [O III] is a powerful and productive technique when studying global nebular properties. Although there is a degeneracy between optically thin and weakly ionized regions when using only two radially varying ions, the technique works well in the aggregate, and the degeneracy is resolved with observations of three sensitive ions. It may be possible to develop similar methods using emission from polycyclic aromatic hydrocarbons, which are easily destroyed in ionized gas and enhanced by non-ionizing UV light in ionization fronts.

Our application of IPM uses the [S II], [O III], and $\mathrm{H} \alpha$ data of the LMC and SMC from the MCELS survey. First, we used $[\mathrm{S} \mathrm{II}] /[\mathrm{O} \mathrm{III}]$ ratio maps to define new boundaries for photoionized $\mathrm{H}$ II regions. The $\left[\mathrm{S}_{\mathrm{II}}\right] /[\mathrm{O} \mathrm{III}]$ maps reveal the nebular ionization structure, thereby allowing us to isolate the emission from individual photoionized H II regions, even if they are overlapping and/or embedded in large complexes or bright DIG. We used these data, together with the $\mathrm{H} \alpha$ surface brightness, to define the boundaries of $401 \mathrm{H}$ II regions in the LMC and 214 in the SMC. The resulting H II region LFs are consistent with those published for these same galaxies (Kennicutt et al. 1989), indicating that the simpler, H $\alpha$-only boundary criteria do result in statistical properties that are similar to those for objects defined by our more physically motivated criteria.

Based on their observed ionization structures, the optical depths of the individual $\mathrm{H}$ II regions were crudely divided into optically thin, optically thick, and blister classes. Based on our models, we assign $f_{\text {esc }}=0.6$ for the population of optically thin regions, 0.3 for blisters, and 0.0 for the optically thick objects. These estimates agree within $23 \%$ with more direct measurements of the optical depth for a sample of objects with known spectral classifications for the ionizing stars.

These rough optical depth classes already yield fundamental new insights into the quantitative radiation transfer of the nebular population and DIG ionization in these galaxies. We find that the frequency of optically thin nebulae is $40 \%$ in the LMC and 33\% in the SMC. The luminosity distributions reveal that the median luminosity of optically thin nebulae is significantly brighter than for those which are optically thick, 


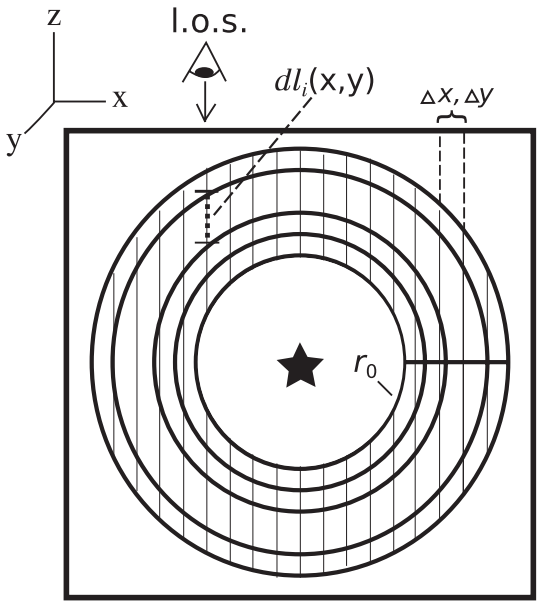

(a) Sphere

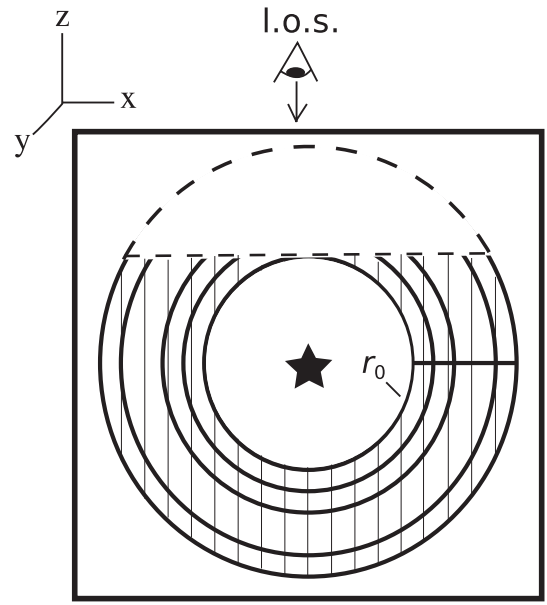

(b) Truncated Sphere

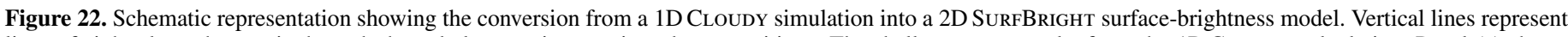

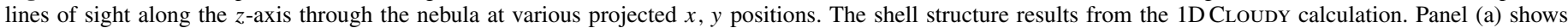
spherically symmetric geometry, and panel (b) shows an object with truncated spherical geometry.

by a factor of two to five. More importantly, the frequency of optically thin nebulae increases with $L$, such that above $\log L /\left(\mathrm{erg} \mathrm{s}^{-1}\right)=37.0$, H II regions in both galaxies are dominated by optically thin objects. Due to their high luminosity and significant $f_{\text {esc }}$, these objects also dominate the total ionizing radiation leaking into the DIG. It will be important to determine whether all star-forming galaxies show a similar luminosity threshold for the dominance of optically thin objects.

We also see a correlation in the frequency of optically thick regions and $\mathrm{H}$ I column density, with the median $N(\mathrm{HI})$ of optically thick nebulae 1.5 and 1.2 times higher than those of optically thin ones in the LMC and SMC, respectively. In contrast, the median $N(\mathrm{HI})$ of all objects measured in the SMC is 2.4 times higher than in the LMC, probably owing to projection effects. It is surprising that despite major differences in the character of the ambient neutral ISM outside of $\mathrm{H}$ II regions, the quantitative properties of the LyC radiative transfer within the nebulae are remarkably similar between the two galaxies. This brings us to an important conclusion: the large-scale fate of ionizing radiation emitted by $\mathrm{O}$ stars in the LMC and SMC may be determined by the external, neutral $\mathrm{H}$ I environment, which in the SMC appears more efficient at trapping radiation once it escapes optically thin $\mathrm{H}$ II regions (e.g., the southwest region of the SMC).

Optically thin nebulae are sufficiently luminous to maintain the ionization of the DIG in both galaxies, as measured by Kennicutt et al. (1995). We also consider the global escape fraction of ionizing radiation from these galaxies into the IGM. We find evidence that luminous, optically thin $\mathrm{H}$ II regions near the outer edges of both galaxies may produce ionizing radiation that escapes into the interstellar environment. This is evidenced by the kiloparsec-scale path lengths traveled by ionizing photons from these massive $\mathrm{H}$ II regions, shown by the existence of extended [O III] halos opening toward the IGM.

We find the combined, luminosity-weighted, LyC escape fractions from all $\mathrm{H}$ II regions to be at least 0.42 and 0.40 in the LMC and SMC, respectively. The corresponding escape luminosities are at least $\log L_{\mathrm{esc}} /\left(\mathrm{erg} \mathrm{s}^{-1}\right)=40.1$ and 39.2 in the LMC and SMC, respectively. Considering the existence of field $\mathrm{O}$ stars with no nebulae, the implied total available LyC luminosity is greater than needed to explain the DIG emission in both galaxies. These are still crude estimates, but an excess implies that a fraction of the ionizing radiation produced leaves the galaxy and may enter the IGM. We currently estimate lower limits to the galactic escape fractions of $f_{\text {esc, gal }}=4 \%-9 \%$ in the SMC, and $11 \%-17 \%$ in the LMC. These values are consistent with $f_{\text {esc gal }} \sim 10 \%-20 \%$, as required for cosmic reionization to be driven by star-forming galaxies at high redshift (Sokasian et al. 2003). These estimates for $f_{\text {esc,gal }}$ would increase when accounting for lower optical depth due to the absence of dust and metals at high redshift.

We thank Snežana Stanimirović and Sungeun Kim for access to the SMC and LMC $N(\mathrm{HI})$ data, respectively. We thank the anonymous referee for helpful contributions. We also thank Joel Lamb and Mark Reynolds for help checking this manuscript. M.S.O., E.W.P., and J.Z. acknowledge support from NSF grant AST-0806476 and a Margaret and Herman Sokol Faculty Award to M.S.O. P.F.W. acknowledges support from the NSF through grant AST-0908566, and A.E.J. acknowledges an NSF Graduate Research Fellowship.

\section{APPENDIX A}

\section{SURFBRIGHT: MODELING SURFACE BRIGHTNESS USING CLOUDY}

The comparison of one-dimensional (1D) photoionization models to observations is most commonly done with spatially integrated emission-line fluxes. This approach is used because a direct comparison to the observed surface-brightness profile of a nebula involves the convolution of nebular geometry with a changing volume emissivity. While computing predicted surface brightnesses is more difficult, it does provide important additional constraints on a model.

We developed the Perl routine SurfBright, which calculates the projected 2D surface brightness of isotropically emitted emission lines in a nebula. This code uses the results of 1D Cloudy simulations and a user-specified 3D geometry. The code works in Cartesian coordinates relative to the nebula with $\mathbf{z}$ parallel to the line of sight from observer to object. This geometry is defined at each $x, y$ coordinate (projected on the sky) by the minimum and maximum radial distance of the cloud along the line of sight, $z_{\min }(x, y)$ and $z_{\max }(x, y)$. A specified physical geometry allows for the prediction of absolute nebular 
Table 5

MCELS LMC H II Region Catalog

\begin{tabular}{|c|c|c|c|c|c|c|}
\hline Object ID & Other ID & $\begin{array}{l}\text { R.A. (J2000) } \\
\text { (h:m:s) }\end{array}$ & $\begin{array}{l}\text { Decl. } \\
\text { (d:m:s) }\end{array}$ & Type $^{\mathrm{b}}$ & $\begin{array}{c}N(\mathrm{HI}) \\
\left(10^{21} \mathrm{~cm}^{-2}\right)\end{array}$ & $\begin{array}{c}L \\
\left(\operatorname{erg~s}^{-1}\right)\end{array}$ \\
\hline MCELS-L1 & $\ldots$ & $04: 44: 59.712$ & $-69: 03: 21.77$ & 2 & 1.75 & 35.12 \\
\hline MCELS-L2 & $\ldots$ & $04: 45: 04.784$ & $-69: 04: 24.94$ & 2 & 1.90 & 35.27 \\
\hline MCELS-L3 & DEM L1 & $04: 47: 19.484$ & $-69: 18: 27.33$ & 2 & 1.89 & 36.41 \\
\hline MCELS-L4 & DEM L2 & 04:48:53.701 & $-69: 09: 37.95$ & 1 & 3.24 & 36.51 \\
\hline MCELS-L5 & DEM L6-3 & 04:49:06.811 & $-69: 20: 25.54$ & 1 & 2.71 & 36.53 \\
\hline MCELS-L6 & DEM L3 & 04:49:07.360 & $-68: 24: 16.55$ & 1 & 2.34 & 36.55 \\
\hline MCELS-L7 & DEM L4-3 & $04: 49: 11.346$ & $-69: 16: 07.76$ & 0 & 3.00 & 36.35 \\
\hline MCELS-L8 & DEM L4-9 & $04: 49: 18.365$ & $-69: 13: 53.73$ & 1 & 3.57 & 35.91 \\
\hline MCELS-L9 & DEM L4-10 & 04:49:18.682 & $-69: 13: 19.99$ & 1 & 3.52 & 35.70 \\
\hline MCELS-L10 & DEM L5 & $04: 49: 24.373$ & $-69: 07: 36.79$ & 3 & 3.24 & 35.90 \\
\hline MCELS-L11 & DEM L4-5 & $04: 49: 25.229$ & $-69: 15: 23.64$ & 1 & 3.29 & $35.85^{\mathrm{c}}$ \\
\hline MCELS-L12 & DEM L4-2 & $04: 49: 25.860$ & $-69: 11: 59.91$ & 3 & 3.08 & 37.08 \\
\hline MCELS-L13 & DEM L4-4 & $04: 49: 30.731$ & $-69: 13: 34.69$ & 3 & 3.49 & $36.66^{\mathrm{c}}$ \\
\hline MCELS-L14 & DEM L4-8 & 04:49:44.439 & $-69: 12: 54.51$ & 1 & 3.54 & 36.02 \\
\hline MCELS-L15 & DEM L6-2 & 04:49:49.192 & $-69: 20: 02.83$ & 3 & 3.31 & 37.46 \\
\hline MCELS-L16 & DEM L4-1 & $04: 49: 51.014$ & $-69: 11: 44.37$ & 3 & 2.97 & $37.36^{\mathrm{d}}$ \\
\hline MCELS-L17 & DEM L4-7 & 04:50:02.415 & $-69: 13: 23.28$ & 1 & 2.94 & $35.80^{\mathrm{d}}$ \\
\hline MCELS-L18 & DEM L4-6 & 04:50:05.934 & $-69: 12: 04.74$ & 1 & 2.98 & $35.81^{\mathrm{c}}$ \\
\hline MCELS-L19 & DEM L7 & 04:50:08.046 & $-67: 42: 10.15$ & 0 & 1.35 & 37.32 \\
\hline MCELS-L20 & DEM L6-1 & $04: 50: 37.795$ & $-69: 25: 28.01$ & 3 & 2.75 & 37.64 \\
\hline MCELS-L21 & $\ldots$ & $04: 51: 32.198$ & $-68: 24: 24.06$ & 3 & 1.53 & 35.84 \\
\hline MCELS-L22 & DEM L8a & $04: 51: 44.200$ & $-66: 55: 13.76$ & 1 & 3.16 & 36.29 \\
\hline MCELS-L23 & DEM L10a-2 & $04: 51: 47.542$ & $-69: 23: 09.55$ & 1 & 2.22 & 37.16 \\
\hline MCELS-L24 & DEM L10a-3 & $04: 51: 49.005$ & $-69: 24: 25.29$ & 3 & 2.00 & 37.16 \\
\hline MCELS-L25 & DEM L10a-1 & $04: 51: 52.784$ & $-69: 23: 29.29$ & 1 & 1.73 & 36.71 \\
\hline MCELS-L26 & DEM L17 & $04: 51: 54.020$ & $-70: 47: 01.43$ & 0 & 1.45 & 36.63 \\
\hline MCELS-L27 & DEM L8b & 04:52:07.354 & $-66: 55: 31.39$ & 3 & 3.15 & 37.41 \\
\hline MCELS-L28 & DEM L8c & 04:52:11.705 & $-66: 54: 28.81$ & 1 & 3.87 & 36.63 \\
\hline MCELS-L29 & DEM L10b-1 & $04: 52: 13.010$ & $-69: 20: 15.75$ & 2 & 3.04 & 37.69 \\
\hline MCELS-L30 & BSDL139 & $04: 52: 17.520$ & $-70: 36: 29.19$ & 0 & 1.51 & 35.56 \\
\hline MCELS-L31 & DEM L9 & $04: 52: 19.371$ & $-68: 24: 39.22$ & 0 & 1.59 & 36.30 \\
\hline MCELS-L32 & $\ldots$ & $04: 52: 23.318$ & $-66: 55: 15.98$ & 1 & 3.02 & 35.62 \\
\hline MCELS-L33 & DEM L10b-2 & $04: 52: 26.500$ & $-69: 21: 44.70$ & 1 & 2.99 & 36.99 \\
\hline MCELS-L34 & DEM L11 & $04: 52: 34.387$ & $-67: 17: 22.56$ & 1 & 1.62 & 37.29 \\
\hline MCELS-L35 & & $04: 52: 35.032$ & $-66: 55: 41.13$ & 1 & 2.45 & 35.82 \\
\hline MCELS-L36 & DEM L15-1 & $04: 52: 46.482$ & $-69: 12: 53.55$ & 1 & 2.73 & 37.12 \\
\hline MCELS-L37 & DEM L14 & $04: 52: 47.119$ & $-68: 54: 40.49$ & 0 & 1.90 & 36.37 \\
\hline MCELS-L38 & DEM L10b-3 & $04: 52: 59.500$ & $-69: 23: 29.50$ & 3 & 2.18 & 37.20 \\
\hline MCELS-L39 & DEM L13 & 04:53:06.281 & $-68: 02: 37.57$ & 2 & 1.57 & 37.60 \\
\hline MCELS-L40 & DEM L15-2 & 04:53:07.055 & $-69: 14: 11.25$ & 3 & 3.21 & 36.64 \\
\hline MCELS-L41 & DEM L12 & $04: 53: 20.950$ & $-66: 56: 18.67$ & 4 & 1.72 & 37.16 \\
\hline MCELS-L42 & DEM L21 & $04: 53: 25.969$ & $-70: 36: 00.14$ & 3 & 1.07 & 36.85 \\
\hline MCELS-L43 & DEM L20 & $04: 53: 30.602$ & $-67: 23: 21.90$ & 1 & 1.34 & 36.23 \\
\hline MCELS-L44 & DEM L18 & $04: 53: 33.254$ & $-67: 03: 29.73$ & 3 & 1.96 & 36.26 \\
\hline MCELS-L45 & DEM L19 & $04: 53: 35.499$ & $-67: 14: 09.07$ & 3 & 1.17 & 36.79 \\
\hline MCELS-L46 & DEM L16 & 04:53:38.798 & $-68: 49: 00.14$ & 0 & 1.28 & 36.67 \\
\hline MCELS-L47 & DEM L22-1 & $04: 53: 57.512$ & $-69: 10: 27.73$ & 1 & 2.80 & 36.64 \\
\hline MCELS-L48 & DEM L25 & 04:53:58.907 & $-70: 00: 52.58$ & 2 & 0.63 & 37.29 \\
\hline MCELS-L49 & DEM L22-2 & 04:54:03.309 & $-69: 12: 08.42$ & 2 & 2.56 & 37.64 \\
\hline MCELS-L50 & DEM L29a & 04:54:05.699 & $-66: 45: 56.84$ & 0 & 2.09 & 35.34 \\
\hline MCELS-L51 & DEM L27 & $04: 54: 10.978$ & $-66: 54: 01.06$ & 0 & 1.51 & 36.64 \\
\hline MCELS-L52 & DEM L26 & 04:54:13.387 & $-68: 21: 52.48$ & 1 & 1.76 & 37.08 \\
\hline MCELS-L53 & DEM L23 & $04: 54: 24.408$ & $-69: 29: 42.54$ & 0 & 3.10 & 35.87 \\
\hline MCELS-L54 & DEM L22-3 & $04: 54: 25.240$ & $-69: 10: 56.81$ & 1 & 2.22 & 36.87 \\
\hline MCELS-L55 & DEM L22-4 & $04: 54: 27.627$ & $-69: 09: 36.65$ & 1 & 2.32 & 37.19 \\
\hline MCELS-L56 & DEM L29b & $04: 54: 27.706$ & $-66: 44: 34.83$ & 2 & 1.49 & 35.36 \\
\hline MCELS-L57 & DEM L30 & $04: 55: 02.964$ & $-67: 15: 52.65$ & 0 & 1.74 & 36.44 \\
\hline MCELS-L58 & DEM L31 & $04: 55: 10.915$ & $-67: 11: 32.12$ & 3 & 1.11 & 37.77 \\
\hline MCELS-L59 & DEM L22-5 & $04: 55: 17.703$ & $-69: 11: 45.04$ & 2 & 2.05 & 37.26 \\
\hline MCELS-L60 & DEM L24 & $04: 55: 25.562$ & $-69: 16: 06.28$ & 1 & 0.99 & 36.06 \\
\hline MCELS-L61 & DEM L32 & $04: 55: 34.509$ & $-68: 25: 38.75$ & 1 & 2.56 & 36.58 \\
\hline MCELS-L62 & DEM L33 & $04: 55: 40.786$ & $-68: 38: 43.30$ & 0 & 1.70 & 37.08 \\
\hline MCELS-L63 & DEM L28+35 & 04:55:58.368 & $-65: 57: 49.86$ & 2 & 1.78 & 36.73 \\
\hline MCELS-L64 & DEM L36 & 04:56:33.538 & $-69: 29: 03.62$ & 0 & 1.07 & 37.55 \\
\hline MCELS-L65 & DEM L34 & $04: 56: 34.254$ & $-66: 26: 51.77$ & 2 & 2.99 & 39.01 \\
\hline
\end{tabular}


Table 5

(Continued)

\begin{tabular}{|c|c|c|c|c|c|c|}
\hline Object ID & Other ID ${ }^{\mathrm{a}}$ & $\begin{array}{l}\text { R.A. (J2000) } \\
\text { (h:m:s) }\end{array}$ & $\begin{array}{l}\text { Decl. } \\
\text { (d:m:s) }\end{array}$ & Type $^{\text {b }}$ & $\begin{array}{c}N(\mathrm{HI}) \\
\left(10^{21} \mathrm{~cm}^{-2}\right)\end{array}$ & $\begin{array}{c}L \\
\left(\operatorname{erg~s}^{-1}\right)\end{array}$ \\
\hline MCELS-L66 & DEM L37 & $04: 56: 58.423$ & $-69: 12: 45.30$ & 1 & 1.91 & 36.61 \\
\hline MCELS-L67 & DEM L38 & 04:57:05.751 & $-68: 44: 57.93$ & 2 & 1.87 & 37.36 \\
\hline MCELS-L68 & DEM L40 & $04: 57: 32.688$ & $-67: 39: 09.73$ & 0 & 1.64 & 36.36 \\
\hline MCELS-L69 & $\ldots$ & $04: 57: 44.015$ & $-66: 15: 30.74$ & 1 & 4.68 & 36.11 \\
\hline MCELS-L70 & DEM L41 & $04: 58: 10.285$ & $-66: 21: 33.66$ & 1 & 4.28 & 37.47 \\
\hline MCELS-L71 & DEM L39 & $04: 58: 29.686$ & $-68: 27: 01.87$ & 2 & 1.12 & 38.12 \\
\hline MCELS-L72 & DEM L42 & $04: 58: 48.391$ & $-66: 11: 37.67$ & 1 & 2.84 & 37.38 \\
\hline MCELS-L73 & $\mathrm{N} 12 \mathrm{~A}$ & 04:58:57.290 & $-66: 13: 57.68$ & 1 & 3.11 & 35.98 \\
\hline MCELS-L74 & DEM L43+48 & 04:58:58.751 & $-65: 40: 27.98$ & 1 & 0.93 & 38.25 \\
\hline MCELS-L75 & DEM L44 & $04: 59: 11.953$ & $-66: 16: 02.89$ & 1 & 3.07 & 36.24 \\
\hline MCELS-L76 & DEM L50 & $04: 59: 45.536$ & $-70: 09: 44.61$ & 4 & 1.28 & 37.37 \\
\hline MCELS-L77 & DEM L45 & 04:59:57.202 & $-67: 56: 47.82$ & 3 & 0.98 & 37.28 \\
\hline MCELS-L78 & DEM L51 & 05:00:00.304 & $-70: 03: 30.12$ & 1 & 0.93 & 36.05 \\
\hline MCELS-L79 & DEM L46 & 05:00:06.625 & $-66: 15: 38.70$ & 1 & 2.23 & 36.91 \\
\hline MCELS-L80 & DEM L47 & $05: 00: 10.254$ & $-66: 05: 37.03$ & 1 & 3.29 & 36.60 \\
\hline MCELS-L81 & DEM L49 & $05: 00: 52.524$ & $-66: 23: 16.45$ & 0 & 2.15 & 35.94 \\
\hline MCELS-L82 & DEM L52 & 05:01:48.040 & $-68: 12: 41.43$ & 0 & 1.63 & 36.00 \\
\hline MCELS-L83 & DEM L55 & $05: 01: 50.450$ & $-70: 38: 36.65$ & 0 & 0.62 & 37.03 \\
\hline MCELS-L84 & DEM L53 & $05: 01: 52.472$ & $-70: 04: 47.90$ & 0 & 0.63 & 36.27 \\
\hline MCELS-L85 & DEM L54 & 05:02:06.984 & $-69: 34: 01.86$ & 0 & 0.72 & 35.53 \\
\hline MCELS-L86 & BSDL453 & 05:02:09.150 & $-66: 40: 17.62$ & 2 & 1.29 & 36.30 \\
\hline MCELS-L87 & DEM L60 & 05:02:12.707 & $-69: 03: 37.57$ & 0 & 2.54 & 36.46 \\
\hline MCELS-L88 & DEM L57 & 05:02:48.614 & $-67: 00: 00.80$ & 0 & 1.82 & 36.56 \\
\hline MCELS-L89 & DEM L61 & 05:03:13.214 & $-65: 57: 24.11$ & 0 & 2.38 & 36.88 \\
\hline MCELS-L90 & DEM L58 & $05: 03: 15.571$ & $-68: 27: 07.27$ & 0 & 1.37 & 36.20 \\
\hline MCELS-L91 & DEM L56 & $05: 03: 22.816$ & $-66: 42: 02.25$ & 0 & 1.34 & 36.42 \\
\hline MCELS-L92 & DEM L59 & 05:04:20.091 & $-67: 18: 34.97$ & 2 & 2.27 & 37.45 \\
\hline MCELS-L93 & DEM L63 & $05: 04: 23.527$ & $-70: 44: 06.97$ & 1 & 1.37 & 37.18 \\
\hline MCELS-L94 & DEM L62 & $05: 04: 25.175$ & $-69: 03: 32.56$ & 0 & 1.86 & 37.23 \\
\hline MCELS-L95 & DEM L64a & $05: 04: 31.355$ & $-70: 54: 06.37$ & 1 & 1.78 & 36.22 \\
\hline MCELS-L96 & DEM L64b & $05: 04: 38.867$ & $-70: 54: 43.46$ & 1 & 2.03 & 36.75 \\
\hline MCELS-L97 & DEM L68-2 & 05:04:39.307 & $-70: 10: 31.78$ & 1 & 1.34 & 35.93 \\
\hline MCELS-L98 & DEM L68-1 & $05: 04: 47.859$ & $-70: 05: 38.53$ & 1 & 2.16 & 35.25 \\
\hline MCELS-L99 & DEM L65 & $05: 04: 48.830$ & $-67: 33: 18.31$ & 2 & 1.94 & 36.84 \\
\hline MCELS-L100 & DEM L67 & 05:04:55.111 & $-70: 07: 40.58$ & 1 & 1.91 & 36.38 \\
\hline MCELS-L101 & DEM L66 & 05:05:00.179 & $-68: 03: 41.41$ & 3 & 1.58 & 37.56 \\
\hline MCELS-L102 & DEM L68 & 05:05:06.741 & $-70: 06: 24.01$ & 3 & 1.29 & $37.51^{\mathrm{d}}$ \\
\hline MCELS-L103 & DEM L70 & $05: 05: 16.650$ & $-68: 05: 40.62$ & 3 & 1.56 & 36.94 \\
\hline MCELS-L104 & DEM L69 & $05: 05: 16.939$ & $-66: 55: 15.29$ & 1 & 2.01 & 36.68 \\
\hline MCELS-L105 & DEM L71 & 05:05:41.007 & $-67: 52: 49.18$ & 0 & 1.63 & 35.58 \\
\hline MCELS-L106 & DEM L72 & 05:06:04.972 & $-65: 41: 28.83$ & 3 & 1.55 & 36.46 \\
\hline MCELS-L107 & DEM L73 & $05: 06: 12.116$ & $-68: 07: 28.47$ & 1 & 2.33 & 37.48 \\
\hline MCELS-L108 & DEM L76 & $05: 06: 45.254$ & $-68: 26: 34.36$ & 3 & 1.52 & 37.51 \\
\hline MCELS-L109 & DEM L74 & $05: 06: 46.312$ & $-68: 09: 53.62$ & 1 & 2.77 & 36.69 \\
\hline MCELS-L110 & DEM L75 & $05: 06: 47.447$ & $-70: 44: 44.35$ & 0 & 0.93 & 37.09 \\
\hline MCELS-L111 & DEM L77 & $05: 06: 50.057$ & $-66: 54: 55.70$ & 0 & 1.24 & 36.65 \\
\hline MCELS-L112 & DEM L78 & 05:06:59.922 & $-67: 56: 46.36$ & 0 & 1.17 & 35.28 \\
\hline MCELS-L113 & DEM L80 & 05:07:19.067 & $-70: 27: 02.39$ & 0 & 0.93 & 37.42 \\
\hline MCELS-L114 & DEM L79 & $05: 07: 21.466$ & $-68: 32: 05.10$ & 1 & 2.12 & 36.76 \\
\hline MCELS-L115 & DEM L81 & $05: 07: 37.534$ & $-71: 10: 31.96$ & 0 & 0.91 & 36.10 \\
\hline MCELS-L116 & DEM L83 & $05: 07: 39.283$ & $-71: 01: 30.45$ & 0 & 0.94 & 35.76 \\
\hline MCELS-L117 & DEM L84 & 05:08:42.555 & $-68: 45: 51.94$ & 4 & 1.83 & 37.86 \\
\hline MCELS-L118 & DEM L85 & $05: 09: 24.026$ & $-68: 45: 44.00$ & 1 & 3.14 & 36.76 \\
\hline MCELS-L119 & DEM L86 & $05: 09: 34.253$ & $-68: 53: 45.05$ & 3 & 2.55 & 38.13 \\
\hline MCELS-L120 & DEM L91 & 05:09:38.133 & $-71: 26: 21.73$ & 0 & 1.05 & 36.29 \\
\hline MCELS-L121 & DEM L89 & 05:09:39.490 & $-67: 55: 14.32$ & 3 & 1.86 & 37.45 \\
\hline MCELS-L122 & DEM L88 & 05:09:53.010 & $-68: 29: 11.15$ & 1 & 2.37 & 37.10 \\
\hline MCELS-L123 & DEM L90 & $05: 10: 23.013$ & $-67: 09: 27.18$ & 0 & 2.06 & 36.32 \\
\hline MCELS-L124 & DEM L92 & 05:10:30.269 & $-69: 25: 56.41$ & 0 & 1.38 & 36.56 \\
\hline MCELS-L125 & DEM L93 & $05: 10: 43.482$ & $-67: 04: 50.36$ & 1 & 2.03 & 36.05 \\
\hline MCELS-L126 & DEM L95 & $05: 10: 54.259$ & $-69: 03: 08.93$ & 0 & 2.33 & 35.57 \\
\hline MCELS-L127 & DEM L94 & $05: 10: 59.381$ & $-67: 07: 34.23$ & 1 & 1.66 & 36.18 \\
\hline MCELS-L128 & DEM L96 & $05: 11: 23.627$ & $-69: 03: 53.95$ & 1 & 2.71 & 35.76 \\
\hline MCELS-L129 & DEM L97 & 05:12:07.699 & $-67: 06: 54.13$ & 0 & 2.88 & 36.96 \\
\hline MCELS-L130 & DEM L101-1 & $05: 12: 08.223$ & $-70: 28: 42.91$ & 1 & 2.50 & 35.70 \\
\hline
\end{tabular}


Table 5

(Continued)

\begin{tabular}{|c|c|c|c|c|c|c|}
\hline Object ID & Other ID ${ }^{\mathrm{a}}$ & $\begin{array}{l}\text { R.A. (J2000) } \\
\text { (h:m:s) }\end{array}$ & $\begin{array}{l}\text { Decl. } \\
\text { (d:m:s) }\end{array}$ & Type $^{\mathrm{b}}$ & $\begin{array}{c}N(\mathrm{H} \mathrm{I}) \\
\left(10^{21} \mathrm{~cm}^{-2}\right)\end{array}$ & $\begin{array}{c}L \\
\left(\operatorname{erg~s}^{-1}\right)\end{array}$ \\
\hline MCELS-L131 & DEM L100-1 & 05:12:14.999 & $-70: 28: 04.74$ & 1 & 2.03 & 35.91 \\
\hline MCELS-L132 & DEM L100-2 & $05: 12: 21.944$ & $-70: 27: 33.34$ & 1 & 1.87 & 35.33 \\
\hline MCELS-L133 & DEM L98 & $05: 12: 24.498$ & $-67: 15: 40.16$ & 0 & 2.41 & 36.37 \\
\hline MCELS-L134 & DEM L101-2 & $05: 12: 28.366$ & $-70: 24: 52.27$ & 3 & 2.61 & 36.52 \\
\hline MCELS-L135 & DEM L101-3 & $05: 12: 30.176$ & $-70: 24: 21.63$ & 1 & 2.22 & 34.99 \\
\hline MCELS-L136 & DEM L101-3 & $05: 12: 30.397$ & $-70: 25: 21.72$ & 1 & 2.17 & 35.14 \\
\hline MCELS-L137 & DEM L102 & $05: 12: 44.181$ & $-70: 22: 02.13$ & 2 & 2.22 & 36.14 \\
\hline MCELS-L138 & DEM L99 & $05: 12: 48.730$ & $-67: 02: 18.21$ & 1 & 2.31 & 36.29 \\
\hline MCELS-L139 & DEM L103 & $05: 13: 07.132$ & $-69: 01: 56.31$ & 0 & 2.27 & 36.64 \\
\hline MCELS-L140 & DEM L104-1 & $05: 13: 10.428$ & $-69: 22: 34.73$ & 1 & 2.81 & 37.26 \\
\hline MCELS-L141 & N30D & $05: 13: 16.280$ & $-67: 28: 24.37$ & 1 & 2.94 & 36.17 \\
\hline MCELS-L142 & DEM L111 & 05:13:18.709 & $-71: 22: 05.07$ & 0 & 1.25 & 35.98 \\
\hline MCELS-L143 & DEM L104-2 & $05: 13: 18.995$ & $-69: 21: 25.64$ & 1 & 2.76 & 37.45 \\
\hline MCELS-L144 & DEM L104-3 & $05: 13: 21.433$ & $-69: 22: 37.56$ & 1 & 2.60 & 36.68 \\
\hline MCELS-L145 & DEM L109 & $05: 13: 25.389$ & $-69: 10: 55.22$ & 0 & 2.15 & 36.29 \\
\hline MCELS-L146 & DEM L108 & $05: 13: 34.910$ & $-69: 17: 05.90$ & 2 & 2.48 & 37.76 \\
\hline MCELS-L147 & DEM L104-4 & $05: 13: 40.515$ & $-69: 22: 30.42$ & 2 & 1.60 & 37.19 \\
\hline MCELS-L148 & DEM L105 & $05: 13: 43.423$ & $-67: 22: 42.83$ & 2 & 1.66 & 37.53 \\
\hline MCELS-L149 & DEM L104-5 & $05: 13: 48.499$ & $-69: 23: 14.82$ & 1 & 1.77 & 36.81 \\
\hline MCELS-L150 & DEM L106 & $05: 13: 53.149$ & $-67: 27: 05.10$ & 2 & 2.93 & 37.56 \\
\hline MCELS-L151 & DEM L110 & $05: 14: 01.760$ & $-69: 31: 54.85$ & 2 & 1.30 & 37.37 \\
\hline MCELS-L152 & DEM L107+115 & 05:14:05.973 & $-67: 09: 01.65$ & 3 & 1.75 & 37.52 \\
\hline MCELS-L153 & DEM L114 & $05: 14: 08.156$ & $-70: 07: 42.59$ & 0 & 1.68 & 35.54 \\
\hline MCELS-L154 & DEM L112 & $05: 14: 34.403$ & $-67: 34: 02.61$ & 0 & 2.52 & 36.78 \\
\hline MCELS-L155 & DEM L119 & 05:14:53.107 & $-71: 37: 09.36$ & 3 & 1.21 & 36.83 \\
\hline MCELS-L156 & DEM L113 & $05: 14: 53.777$ & $-69: 25: 58.19$ & 0 & 1.60 & 37.44 \\
\hline MCELS-L157 & DEM L116 & 05:15:07.167 & $-66: 28: 06.01$ & 1 & 1.07 & 36.29 \\
\hline MCELS-L158 & DEM L120 & $05: 15: 20.451$ & $-69: 43: 29.81$ & 0 & 1.36 & 36.04 \\
\hline MCELS-L159 & DEM L117 & $05: 15: 34.094$ & $-67: 20: 34.67$ & 0 & 1.77 & 35.73 \\
\hline MCELS-L160 & DEM L118 & $05: 15: 45.680$ & $-66: 42: 48.98$ & 0 & 1.36 & 36.17 \\
\hline MCELS-L161 & DEM L124 & $05: 16: 53.145$ & $-69: 53: 01.24$ & 0 & 2.02 & 36.21 \\
\hline MCELS-L162 & DEM L121 & $05: 16: 53.381$ & $-67: 19: 51.03$ & 1 & 2.20 & 36.90 \\
\hline MCELS-L163 & & $05: 16: 53.563$ & $-66: 00: 23.33$ & 2 & 1.07 & 35.84 \\
\hline MCELS-L164 & DEM L122 & 05:17:06.169 & $-68: 52: 35.45$ & 0 & 3.39 & 35.05 \\
\hline MCELS-L165 & DEM L131 & $05: 17: 39.052$ & $-71: 15: 31.94$ & 2 & 1.72 & 37.35 \\
\hline MCELS-L166 & DEM L130 & $05: 17: 44.512$ & $-69: 24: 00.25$ & 0 & 2.34 & 36.96 \\
\hline MCELS-L167 & DEM L128 & $05: 17: 45.970$ & $-68: 47: 03.99$ & 1 & 3.54 & 36.13 \\
\hline MCELS-L168 & DEM L129 & 05:17:47.999 & $-67: 54: 05.00$ & 2 & 1.83 & 36.19 \\
\hline MCELS-L169 & DEM L127 & $05: 17: 48.973$ & $-67: 20: 58.24$ & 2 & 1.28 & 37.01 \\
\hline MCELS-L170 & DEM L125 & $05: 17: 51.000$ & $-66: 01: 16.99$ & 1 & 1.45 & 37.11 \\
\hline MCELS-L171 & DEM L123+132 & $05: 17: 59.694$ & $-69: 11: 00.83$ & 2 & 2.23 & 38.54 \\
\hline MCELS-L172 & DEM L126 & $05: 18: 16.646$ & $-65: 57: 40.89$ & 0 & 1.67 & 36.49 \\
\hline MCELS-L173 & DEM L134-3 & $05: 18: 27.369$ & $-69: 39: 54.84$ & 1 & 2.81 & 36.85 \\
\hline MCELS-L174 & & $05: 18: 40.581$ & $-67: 05: 21.22$ & 3 & 1.29 & 36.32 \\
\hline MCELS-L175 & DEM L134-4 & $05: 18: 43.692$ & $-69: 39: 15.27$ & 1 & 2.14 & 36.83 \\
\hline MCELS-L176 & DEM L134-1+133 & $05: 19: 16.370$ & $-69: 38: 42.71$ & 2 & 1.47 & 38.09 \\
\hline MCELS-L177 & DEM L134-2 & 05:19:35.777 & $-69: 38: 46.80$ & 3 & 2.25 & 36.32 \\
\hline MCELS-L178 & DEM L135 & $05: 19: 48.280$ & $-65: 53: 53.17$ & 0 & 0.91 & 37.38 \\
\hline MCELS-L179 & DEM L139 & 05:20:19.421 & $-66: 28: 54.36$ & 0 & 1.40 & 35.37 \\
\hline MCELS-L180 & DEM L136 & $05: 20: 22.132$ & $-66: 53: 41.66$ & 2 & 1.61 & 36.89 \\
\hline MCELS-L181 & DEM L141 & 05:20:30.647 & $-68: 01: 02.02$ & 1 & 3.59 & 36.14 \\
\hline MCELS-L182 & DEM L138 & $05: 20: 34.007$ & $-66: 46: 37.94$ & 2 & 2.02 & 37.03 \\
\hline MCELS-L183 & & $05: 20: 40.327$ & $-66: 48: 46.44$ & 0 & 2.40 & 35.10 \\
\hline MCELS-L184 & DEM L137 & $05: 20: 48.691$ & $-65: 27: 24.64$ & 3 & 0.77 & 37.64 \\
\hline MCELS-L185 & DEM L146 & $05: 20: 54.450$ & $-71: 43: 17.48$ & 0 & 1.99 & 35.66 \\
\hline MCELS-L186 & $\ldots$ & $05: 21: 04.863$ & $-71: 41: 42.66$ & 0 & 1.95 & 35.02 \\
\hline MCELS-L187 & $\ldots$ & $05: 21: 17.438$ & $-66: 47: 13.89$ & 0 & 2.31 & 35.37 \\
\hline MCELS-L188 & DEM L145 & $05: 21: 19.322$ & $-69: 40: 50.04$ & 1 & 2.42 & 36.71 \\
\hline MCELS-L189 & DEM L147 & $05: 21: 26.277$ & $-69: 56: 50.29$ & 0 & 1.81 & 35.91 \\
\hline MCELS-L190 & DEM L143 & $05: 21: 26.923$ & $-68: 52: 04.13$ & 0 & 1.87 & 36.86 \\
\hline MCELS-L191 & DEM L144 & $05: 21: 31.101$ & $-68: 10: 47.80$ & 2 & 3.94 & 35.94 \\
\hline MCELS-L192 & DEM L149 & $05: 21: 34.561$ & $-69: 40: 26.84$ & 1 & 3.01 & 37.15 \\
\hline MCELS-L193 & DEM L150 & $05: 21: 36.618$ & $-67: 46: 33.56$ & 1 & 2.90 & 36.24 \\
\hline MCELS-L194 & DEM L140 & $05: 21: 37.132$ & $-67: 54: 49.07$ & 1 & 3.06 & 37.46 \\
\hline MCELS-L195 & DEM L148 & $05: 21: 37.250$ & $-69: 59: 37.00$ & 0 & 2.10 & 36.40 \\
\hline
\end{tabular}


Table 5

(Continued)

\begin{tabular}{|c|c|c|c|c|c|c|}
\hline Object ID & Other ID ${ }^{\mathrm{a}}$ & $\begin{array}{l}\text { R.A. (J2000) } \\
\text { (h:m:s) }\end{array}$ & $\begin{array}{l}\text { Decl. } \\
\text { (d:m:s) }\end{array}$ & Type $^{\text {b }}$ & $\begin{array}{c}N(\mathrm{H} \mathrm{I}) \\
\left(10^{21} \mathrm{~cm}^{-2}\right)\end{array}$ & $\begin{array}{c}L \\
\left(\mathrm{erg} \mathrm{s}^{-1}\right)\end{array}$ \\
\hline MCELS-L196 & DEM L151a & 05:21:46.789 & $-67: 53: 41.51$ & 0 & 4.44 & 36.80 \\
\hline MCELS-L197 & DEM L153 & $05: 21: 49.737$ & $-69: 41: 08.86$ & 1 & 3.15 & 35.85 \\
\hline MCELS-L198 & DEM L151 & $05: 21: 49.869$ & $-67: 51: 45.99$ & 0 & 3.01 & 37.39 \\
\hline MCELS-L199 & DEM L142 & $05: 21: 53.904$ & $-65: 43: 49.45$ & 0 & 0.97 & 37.18 \\
\hline MCELS-L200 & DEM L154 & 05:22:00.937 & $-65: 58: 22.24$ & 2 & 0.92 & 37.57 \\
\hline MCELS-L201 & DEM L152 & $05: 22: 06.859$ & $-67: 56: 46.11$ & 3 & 3.97 & 38.19 \\
\hline MCELS-L202 & DEM L164 & $05: 22: 13.132$ & $-71: 26: 09.80$ & 2 & 1.25 & 37.59 \\
\hline MCELS-L203 & DEM L157 & $05: 22: 16.276$ & $-68: 38: 58.35$ & 1 & 1.68 & 36.61 \\
\hline MCELS-L204 & DEM L158-1 & $05: 22: 19.405$ & $-68: 04: 25.93$ & 1 & 4.89 & 36.64 \\
\hline MCELS-L205 & DEM L155 & $05: 22: 25.482$ & $-65: 44: 46.15$ & 0 & 1.24 & $37.32^{\mathrm{d}}$ \\
\hline MCELS-L206 & DEM L156 & 05:22:26.917 & $-67: 53: 41.49$ & 1 & 4.23 & 37.39 \\
\hline MCELS-L207 & DEM L165 & $05: 22: 27.832$ & $-71: 35: 50.94$ & 1 & 1.88 & 37.57 \\
\hline MCELS-L208 & DEM L158-2 & $05: 22: 29.885$ & $-68: 04: 52.47$ & 1 & 4.45 & 35.93 \\
\hline MCELS-L209 & DEM L163 & $05: 22: 32.269$ & $-70: 08: 45.06$ & 2 & 1.10 & 36.50 \\
\hline MCELS-L210 & DEM L159 & $05: 22: 36.176$ & $-68: 08: 32.19$ & 1 & 4.68 & 36.56 \\
\hline MCELS-L211 & $\ldots$ & $05: 22: 36.961$ & $-66: 38: 52.02$ & 2 & 2.70 & 35.46 \\
\hline MCELS-L212 & DEM L160 & $05: 22: 45.238$ & $-68: 04: 00.00$ & 1 & 4.51 & 37.89 \\
\hline MCELS-L213 & DEM L161 & $05: 22: 47.991$ & $-66: 41: 05.67$ & 1 & 3.15 & 36.38 \\
\hline MCELS-L214 & DEM L168 & $05: 22: 52.698$ & $-69: 50: 57.96$ & 0 & 2.20 & 36.40 \\
\hline MCELS-L215 & DEM L155a & $05: 22: 53.603$ & $-65: 43: 02.54$ & 1 & 1.54 & 36.88 \\
\hline MCELS-L216 & DEM L166a & $05: 23: 06.017$ & $-68: 00: 16.50$ & 1 & 3.74 & 36.28 \\
\hline MCELS-L217 & DEM L162 & 05:23:06.777 & $-66: 22: 32.41$ & 1 & 1.97 & 36.52 \\
\hline MCELS-L218 & DEM L166b & $05: 23: 12.509$ & $-68: 00: 18.30$ & 1 & 4.23 & 36.55 \\
\hline MCELS-L219 & DEM L167 & $05: 23: 16.463$ & $-67: 56: 14.25$ & 1 & 3.53 & 36.65 \\
\hline MCELS-L220 & DEM L170 & $05: 23: 27.358$ & $-68: 12: 24.56$ & 0 & 2.55 & 36.10 \\
\hline MCELS-L221 & DEM L171 & $05: 23: 33.510$ & $-69: 38: 51.00$ & 0 & 2.04 & 36.60 \\
\hline MCELS-L222 & DEM L169 & $05: 23: 39.514$ & $-68: 00: 42.93$ & 1 & 3.51 & 36.26 \\
\hline MCELS-L223 & DEM L172 & $05: 23: 40.552$ & $-69: 37: 00.93$ & 2 & 1.00 & 36.91 \\
\hline MCELS-L224 & DEM L173-3 & $05: 23: 50.817$ & $-69: 41: 25.84$ & 0 & 2.66 & 36.26 \\
\hline MCELS-L225 & DEM L176a & $05: 24: 03.034$ & $-68: 56: 20.67$ & 0 & 2.47 & 35.34 \\
\hline MCELS-L226 & DEM L173-2 & 05:24:04.092 & $-69: 40: 17.65$ & 1 & 3.19 & 36.08 \\
\hline MCELS-L227 & DEM L173-1 & $05: 24: 06.969$ & $-69: 38: 43.07$ & 1 & 2.18 & 36.05 \\
\hline MCELS-L228 & DEM L176b & $05: 24: 09.445$ & $-68: 55: 52.81$ & 0 & 2.46 & 35.24 \\
\hline MCELS-L229 & DEM L174-1 & $05: 24: 12.288$ & $-68: 30: 08.91$ & 1 & 2.76 & 36.31 \\
\hline MCELS-L230 & N132B & $05: 24: 17.526$ & $-69: 38: 55.93$ & 1 & 2.09 & 35.95 \\
\hline MCELS-L231 & DEM L174 & $05: 24: 22.662$ & $-68: 31: 33.90$ & 3 & 2.21 & $37.56^{d}$ \\
\hline MCELS-L232 & DEM L175 & $05: 24: 24.122$ & $-66: 14: 29.44$ & 2 & 2.69 & 37.30 \\
\hline MCELS-L233 & DEM L194 & $05: 24: 28.191$ & $-71: 38: 35.64$ & 0 & 2.16 & 36.00 \\
\hline MCELS-L234 & DEM L178 & $05: 24: 34.185$ & $-69: 27: 09.09$ & 0 & 1.15 & 36.92 \\
\hline MCELS-L235 & DEM L177 & $05: 24: 36.885$ & $-69: 06: 54.62$ & 0 & 1.76 & 38.32 \\
\hline MCELS-L236 & DEM L182+184 & $05: 24: 38.462$ & $-66: 57: 13.08$ & 0 & 1.51 & 37.43 \\
\hline MCELS-L237 & DEM L179 & $05: 24: 39.239$ & $-68: 28: 47.11$ & 1 & 3.24 & 36.26 \\
\hline MCELS-L238 & DEM L180 & $05: 24: 58.237$ & $-68: 28: 41.12$ & 2 & 3.01 & 37.23 \\
\hline MCELS-L239 & DEM L175a & $05: 24: 58.520$ & $-66: 26: 03.38$ & 3 & 3.83 & 37.17 \\
\hline MCELS-L240 & DEM L186 & $05: 25: 02.359$ & $-69: 38: 33.97$ & 3 & 2.03 & 36.26 \\
\hline MCELS-L241 & DEM L188 & $05: 25: 06.099$ & $-71: 27: 48.70$ & 2 & 1.58 & 35.98 \\
\hline MCELS-L242 & DEM L181 & $05: 25: 21.702$ & $-66: 02: 45.50$ & 2 & 2.56 & 37.17 \\
\hline MCELS-L243 & DEM L187 & $05: 25: 25.372$ & $-69: 26: 09.88$ & 0 & 1.00 & 36.91 \\
\hline MCELS-L244 & DEM L183 & $05: 25: 27.356$ & $-66: 21: 52.11$ & 1 & 3.98 & 36.07 \\
\hline MCELS-L245 & DEM L185 & $05: 25: 54.458$ & $-65: 55: 53.12$ & 0 & 2.34 & 35.94 \\
\hline MCELS-L246 & DEM L191 & $05: 26: 01.278$ & $-66: 04: 57.00$ & 4 & 3.91 & 37.19 \\
\hline MCELS-L247 & DEM L189-1 & $05: 26: 04.121$ & $-66: 15: 46.76$ & 0 & 4.41 & 37.67 \\
\hline MCELS-L248 & DEM L193 & 05:26:09.991 & $-67: 10: 37.36$ & 3 & 1.43 & 37.31 \\
\hline MCELS-L249 & DEM L195 & $05: 26: 12.708$ & $-66: 21: 46.89$ & 0 & 1.75 & 37.48 \\
\hline MCELS-L250 & DEM L192+201 & $05: 26: 13.111$ & $-67: 29: 56.09$ & 1 & 1.90 & 38.52 \\
\hline MCELS-L251 & DEM L196-2 & $05: 26: 13.873$ & $-67: 37: 12.77$ & 1 & 2.27 & 36.38 \\
\hline MCELS-L252 & DEM L196-1 & $05: 26: 20.683$ & $-67: 37: 54.64$ & 1 & 1.86 & 37.60 \\
\hline MCELS-L253 & DEM L189-2 & $05: 26: 23.231$ & $-66: 14: 30.93$ & 2 & 4.40 & 36.28 \\
\hline MCELS-L254 & DEM L197 & $05: 26: 28.588$ & $-69: 18: 57.52$ & 3 & 0.78 & 37.38 \\
\hline MCELS-L255 & DEM L196-3 & $05: 26: 28.611$ & $-67: 42: 00.51$ & 2 & 2.07 & 35.98 \\
\hline MCELS-L256 & DEM L198 & $05: 26: 32.213$ & $-69: 02: 03.52$ & 0 & 1.20 & 37.77 \\
\hline MCELS-L257 & DEM L196-4 & $05: 26: 37.867$ & $-67: 43: 27.72$ & 1 & 2.58 & 36.00 \\
\hline MCELS-L258 & DEM L199 & $05: 26: 42.482$ & $-68: 49: 34.13$ & 3 & 1.18 & 38.36 \\
\hline MCELS-L259 & DEM L202 & $05: 27: 10.252$ & $-71: 32: 06.20$ & 2 & 1.99 & 37.57 \\
\hline MCELS-L260 & DEM L208 & $05: 27: 17.601$ & $-70: 34: 46.24$ & 3 & 1.01 & 37.73 \\
\hline
\end{tabular}


Table 5

(Continued)

\begin{tabular}{|c|c|c|c|c|c|c|}
\hline Object ID & Other ID ${ }^{\mathrm{a}}$ & $\begin{array}{l}\text { R.A. (J2000) } \\
\text { (h:m:s) }\end{array}$ & $\begin{array}{l}\text { Decl. } \\
\text { (d:m:s) }\end{array}$ & Type $^{\text {b }}$ & $\begin{array}{c}N(\mathrm{H} \mathrm{I}) \\
\left(10^{21} \mathrm{~cm}^{-2}\right)\end{array}$ & $\begin{array}{c}L \\
\left(\mathrm{erg} \mathrm{s}^{-1}\right)\end{array}$ \\
\hline MCELS-L261 & DEM L206 & $05: 27: 26.753$ & $-71: 23: 27.64$ & 2 & 3.09 & 36.01 \\
\hline MCELS-L262 & $\ldots$ & $05: 27: 31.278$ & $-67: 27: 31.34$ & 0 & 2.18 & 37.51 \\
\hline MCELS-L263 & DEM L203 & $05: 27: 31.358$ & $-68: 26: 59.64$ & 0 & 1.68 & 38.38 \\
\hline MCELS-L264 & DEM L207 & $05: 27: 41.552$ & $-71: 24: 46.37$ & 1 & 2.47 & 36.26 \\
\hline MCELS-L265 & DEM L204 & $05: 27: 54.235$ & $-65: 50: 09.56$ & 4 & 0.65 & 36.49 \\
\hline MCELS-L266 & DEM L209 & $05: 28: 02.218$ & $-69: 21: 36.01$ & 0 & 0.87 & 37.63 \\
\hline MCELS-L267 & DEM L205 & 05:28:06.188 & $-67: 26: 49.96$ & 2 & 2.56 & 37.59 \\
\hline MCELS-L268 & BSDL1844 & $05: 28: 14.614$ & $-67: 23: 57.34$ & 1 & 2.50 & 36.67 \\
\hline MCELS-L269 & DEM L210 & $05: 28: 19.243$ & $-69: 01: 20.89$ & 0 & 1.52 & 37.96 \\
\hline MCELS-L270 & DEM L213 & $05: 28: 43.076$ & $-70: 20: 20.34$ & 0 & 1.44 & 36.44 \\
\hline MCELS-L271 & DEM L211 & $05: 28: 54.271$ & $-67: 43: 23.67$ & 0 & 1.58 & 36.57 \\
\hline MCELS-L272 & DEM L214 & $05: 29: 17.376$ & $-66: 57: 20.69$ & 0 & 0.69 & 36.95 \\
\hline MCELS-L273 & DEM L215 & $05: 29: 33.407$ & $-69: 48: 48.12$ & 0 & 1.23 & 37.26 \\
\hline MCELS-L274 & $\ldots$ & $05: 29: 52.217$ & $-71: 04: 32.83$ & 1 & 2.17 & 36.56 \\
\hline MCELS-L275 & DEM L216 & 05:30:09.440 & $-69: 45: 08.71$ & 0 & 0.91 & 36.18 \\
\hline MCELS-L276 & DEM L218 & $05: 30: 33.907$ & $-70: 07: 59.41$ & 0 & 1.41 & 37.02 \\
\hline MCELS-L277 & DEM L221 & $05: 30: 33.984$ & $-71: 01: 21.22$ & 1 & 2.02 & 38.43 \\
\hline MCELS-L278 & N206B & 05:30:51.189 & $-71: 08: 01.48$ & 1 & 3.48 & 36.83 \\
\hline MCELS-L279 & DEM L222a & $05: 30: 58.078$ & $-67: 20: 28.18$ & 0 & 1.77 & 35.56 \\
\hline MCELS-L280 & DEM L224 & 05:31:02.981 & $-69: 19: 18.87$ & 0 & 0.93 & 38.37 \\
\hline MCELS-L281 & DEM L222b & $05: 31: 11.571$ & $-67: 22: 46.68$ & 0 & 1.47 & 35.91 \\
\hline MCELS-L282 & DEM L223 & 05:31:17.691 & $-67: 26: 54.31$ & 0 & 1.08 & 36.59 \\
\hline MCELS-L283 & DEM L225 & $05: 31: 49.240$ & $-67: 21: 34.55$ & 0 & 1.43 & 35.47 \\
\hline MCELS-L284 & DEM L226 & 05:32:01.731 & $-68: 40: 24.21$ & 1 & 2.43 & 37.21 \\
\hline MCELS-L285 & DEM L228a & $05: 32: 05.424$ & $-66: 24: 45.52$ & 1 & 1.84 & 36.91 \\
\hline MCELS-L286 & BSDL2224 & $05: 32: 10.557$ & $-68: 39: 04.37$ & 1 & 3.63 & 35.68 \\
\hline MCELS-L287 & DEM L227 & $05: 32: 10.871$ & $-68: 28: 22.50$ & 1 & 3.77 & $38.07^{\mathrm{d}}$ \\
\hline MCELS-L288 & DEM L228b & $05: 32: 13.630$ & $-66: 23: 26.13$ & 1 & 1.42 & 36.89 \\
\hline MCELS-L289 & DEM L219+229 & $05: 32: 19.571$ & $-67: 40: 57.44$ & 4 & 2.04 & 38.29 \\
\hline MCELS-L290 & BSDL2247 & $05: 32: 30.650$ & $-68: 40: 12.79$ & 1 & 3.26 & 36.15 \\
\hline MCELS-L291 & DEM L228 & $05: 32: 32.913$ & $-66: 24: 20.24$ & 2 & 0.87 & $37.98^{\mathrm{d}}$ \\
\hline MCELS-L292 & N148a & $05: 32: 44.003$ & $-68: 24: 27.53$ & 1 & 0.00 & 33.31 \\
\hline MCELS-L293 & DEM L230 & $05: 32: 53.527$ & $-67: 49: 08.83$ & 0 & 1.27 & 35.59 \\
\hline MCELS-L294 & DEM L232 & 05:33:03.933 & $-68: 56: 15.26$ & 0 & 2.05 & 38.37 \\
\hline MCELS-L295 & DEM L231 & $05: 33: 10.610$ & $-67: 42: 45.46$ & 3 & 2.74 & 37.24 \\
\hline MCELS-L296 & DEM L234 & 05:33:39.995 & $-67: 31: 02.56$ & 0 & 1.93 & 38.01 \\
\hline MCELS-L297 & DEM L233 & $05: 33: 43.948$ & $-68: 46: 00.59$ & 1 & 2.68 & 36.56 \\
\hline MCELS-L298 & DEM L238 & 05:34:17.669 & $-70: 33: 35.14$ & 4 & 1.29 & 36.02 \\
\hline MCELS-L299 & DEM L236 & $05: 34: 25.943$ & $-67: 05: 45.69$ & 0 & 0.76 & 36.17 \\
\hline MCELS-L300 & DEM L235 & $05: 34: 31.133$ & $-66: 07: 45.96$ & 1 & 1.19 & 37.24 \\
\hline MCELS-L301 & DEM L258 & $05: 34: 37.451$ & $-68: 12: 37.33$ & 0 & 3.22 & 37.04 \\
\hline MCELS-L302 & DEM L239 & $05: 34: 40.000$ & $-66: 14: 19.20$ & 2 & 0.82 & 37.47 \\
\hline MCELS-L303 & DEM L237 & $05: 34: 40.388$ & $-65: 57: 09.98$ & 2 & 1.83 & 37.00 \\
\hline MCELS-L304 & DEM L242 & $05: 34: 50.971$ & $-69: 31: 22.97$ & 1 & 2.70 & 36.64 \\
\hline MCELS-L305 & DEM L240 & $05: 34: 53.055$ & $-67: 21: 23.80$ & 3 & 0.81 & 36.61 \\
\hline MCELS-L306 & DEM L246 & $05: 35: 04.794$ & $-69: 43: 25.48$ & 2 & 2.58 & 38.51 \\
\hline MCELS-L307 & DEM L241 & $05: 35: 15.548$ & $-67: 34: 04.12$ & 3 & 2.28 & 38.37 \\
\hline MCELS-L308 & DEM L245 & $05: 35: 22.447$ & $-67: 42: 08.21$ & 0 & 1.96 & 37.13 \\
\hline MCELS-L309 & DEM L244 & $05: 35: 28.235$ & $-66: 38: 43.13$ & 0 & 0.52 & 37.42 \\
\hline MCELS-L310 & DEM L243 & $05: 35: 33.576$ & $-66: 02: 25.94$ & 1 & 1.56 & 37.66 \\
\hline MCELS-L311 & N59C & $05: 35: 39.078$ & $-67: 37: 07.03$ & 1 & 3.11 & 36.83 \\
\hline MCELS-L312 & DEM L249 & 05:36:08.300 & $-70: 38: 56.31$ & 0 & 2.66 & 35.99 \\
\hline MCELS-L313 & DEM L248 & 05:36:09.783 & $-69: 31: 53.93$ & 0 & 3.27 & 37.91 \\
\hline MCELS-L314 & DEM L247 & $05: 36: 27.965$ & $-66: 02: 12.46$ & 0 & 1.37 & 36.29 \\
\hline MCELS-L315 & DEM L259 & $05: 36: 30.751$ & $-69: 49: 05.73$ & 1 & 3.75 & 36.72 \\
\hline MCELS-L316 & DEM L250 & $05: 36: 39.751$ & $-67: 26: 50.51$ & 1 & 2.37 & 37.22 \\
\hline MCELS-L317 & DEM L251 & $05: 36: 40.231$ & $-66: 26: 31.23$ & 0 & 2.15 & 36.86 \\
\hline MCELS-L318 & DEM L252 & 05:37:02.710 & $-66: 21: 18.29$ & 1 & 2.08 & 37.62 \\
\hline MCELS-L319 & DEM L255 & 05:37:03.001 & $-66: 39: 46.28$ & 1 & 1.14 & 37.62 \\
\hline MCELS-L320 & DEM L260 & $05: 37: 16.661$ & $-69: 46: 00.04$ & 1 & 5.09 & 36.24 \\
\hline MCELS-L321 & DEM L253 & $05: 37: 17.986$ & $-66: 17: 58.25$ & 1 & 2.45 & 37.10 \\
\hline MCELS-L322 & DEM L256 & $05: 37: 28.886$ & $-66: 27: 51.06$ & 0 & 2.34 & 36.20 \\
\hline MCELS-L323 & DEM L261 & 05:37:43.009 & $-69: 22: 02.62$ & 3 & 3.10 & 38.24 \\
\hline MCELS-L324 & DEM L262 & $05: 37: 50.651$ & $-69: 39: 19.82$ & 0 & 4.32 & 37.65 \\
\hline MCELS-L325 & DEM L254 & $05: 38: 12.463$ & $-66: 18: 16.33$ & 1 & 1.52 & 36.98 \\
\hline
\end{tabular}


Table 5

(Continued)

\begin{tabular}{|c|c|c|c|c|c|c|}
\hline Object ID & Other ID ${ }^{\mathrm{a}}$ & $\begin{array}{l}\text { R.A. (J2000) } \\
\text { (h:m:s) }\end{array}$ & $\begin{array}{l}\text { Decl. } \\
\text { (d:m:s) }\end{array}$ & Type $^{b}$ & $\begin{array}{c}N(\mathrm{H} \mathrm{I}) \\
\left(10^{21} \mathrm{~cm}^{-2}\right)\end{array}$ & $\begin{array}{c}L \\
\left(\mathrm{erg} \mathrm{s}^{-1}\right)\end{array}$ \\
\hline MCELS-L326 & DEM L265 & $05: 38: 18.585$ & $-70: 41: 15.23$ & 2 & 2.82 & 37.37 \\
\hline MCELS-L327 & DEM L264 & $05: 38: 21.623$ & $-66: 35: 24.71$ & 0 & 1.28 & 36.43 \\
\hline MCELS-L328 & DEM L263 & 05:38:36.013 & $-69: 05: 10.80$ & 3 & 4.24 & 39.66 \\
\hline MCELS-L329 & DEM L266 & 05:38:48.193 & $-70: 04: 28.06$ & 0 & 5.47 & 35.43 \\
\hline MCELS-L330 & DEM L269-3 & 05:38:57.582 & $-69: 29: 55.48$ & 0 & 4.73 & 38.27 \\
\hline MCELS-L331 & DEM L267 & $05: 39: 14.137$ & $-70: 12: 38.80$ & 0 & 5.51 & 36.43 \\
\hline MCELS-L332 & N158D & $05: 39: 16.403$ & $-69: 33: 16.72$ & 1 & 4.88 & 36.70 \\
\hline MCELS-L333 & DEM L269-1 & $05: 39: 33.123$ & $-69: 25: 24.81$ & 2 & 3.46 & 38.15 \\
\hline MCELS-L334 & $\ldots$ & 05:39:34.309 & $-69: 39: 23.08$ & 1 & 5.37 & 37.53 \\
\hline MCELS-L335 & DEM L269-2 & $05: 39: 37.420$ & $-69: 28: 07.18$ & 1 & 4.65 & 36.89 \\
\hline MCELS-L336 & DEM L284 & 05:39:46.131 & $-69: 38: 49.01$ & 1 & 4.96 & $38.56^{\mathrm{d}}$ \\
\hline MCELS-L337 & $\ldots$ & 05:39:46.131 & $-69: 38: 49.01$ & 1 & 4.29 & 37.82 \\
\hline MCELS-L338 & DEM L270 & $05: 39: 48.031$ & $-66: 08: 52.19$ & 2 & 1.23 & 36.97 \\
\hline MCELS-L339 & DEM L274-3 & $05: 39: 51.136$ & $-71: 09: 21.74$ & 1 & 5.05 & 35.59 \\
\hline MCELS-L340 & DEM L274-2 & 05:39:53.789 & $-71: 09: 42.85$ & 1 & 5.15 & 36.24 \\
\hline MCELS-L341 & DEM L274-1 & 05:39:58.032 & $-71: 10: 16.21$ & 1 & 5.20 & 36.30 \\
\hline MCELS-L342 & DEM L272 & 05:40:03.397 & $-69: 49: 14.35$ & 1 & 6.54 & 36.02 \\
\hline MCELS-L343 & DEM L271 & $05: 40: 06.813$ & $-69: 45: 28.34$ & 3 & 6.33 & 38.27 \\
\hline MCELS-L344 & DEM L276 & 05:40:09.510 & $-71: 11: 05.44$ & 1 & 5.06 & 36.77 \\
\hline MCELS-L345 & DEM L278 & $05: 40: 10.112$ & $-71: 12: 26.07$ & 1 & 5.46 & 36.02 \\
\hline MCELS-L346 & DEM L275 & $05: 40: 12.281$ & $-69: 55: 00.90$ & 1 & 5.59 & 36.19 \\
\hline MCELS-L347 & DEM L273 & 05:40:13.530 & $-68: 59: 26.26$ & 1 & 4.99 & 36.62 \\
\hline MCELS-L348 & DEM L277 & $05: 40: 22.892$ & $-69: 53: 15.66$ & 1 & 5.76 & 35.82 \\
\hline MCELS-L349 & $\ldots$ & 05:40:23.411 & $-69: 40: 16.72$ & 0 & 4.64 & 37.16 \\
\hline MCELS-L350 & DEM L279 & $05: 40: 26.944$ & $-69: 50: 23.95$ & 0 & 8.43 & 36.02 \\
\hline MCELS-L351 & DEM L281 & $05: 40: 42.047$ & $-70: 02: 31.46$ & 1 & 4.74 & 36.99 \\
\hline MCELS-L352 & $\ldots$ & $05: 40: 43.558$ & $-69: 47: 18.69$ & 2 & 7.14 & 36.12 \\
\hline MCELS-L353 & $\ldots$ & 05:40:48.293 & $-69: 49: 33.33$ & 2 & 7.29 & 36.04 \\
\hline MCELS-L354 & DEM L280 & $05: 40: 48.494$ & $-70: 10: 06.31$ & 1 & 5.33 & 36.06 \\
\hline MCELS-L355 & DEM L283 & $05: 40: 50.828$ & $-69: 46: 07.70$ & 1 & 6.01 & 35.85 \\
\hline MCELS-L356 & DEM L287 & $05: 40: 53.446$ & $-71: 12: 05.45$ & 3 & 5.34 & 36.21 \\
\hline MCELS-L357 & DEM L290 & $05: 40: 57.297$ & $-70: 54: 40.87$ & 1 & 4.39 & 35.79 \\
\hline MCELS-L358 & DEM L282 & $05: 41: 03.003$ & $-69: 55: 24.31$ & 0 & 4.35 & 36.91 \\
\hline MCELS-L359 & DEM L288 & 05:41:07.076 & $-71: 13: 42.95$ & 0 & 4.90 & 35.79 \\
\hline MCELS-L360 & DEM L291 & $05: 41: 12.128$ & $-70: 29: 45.45$ & 0 & 4.71 & 35.80 \\
\hline MCELS-L361 & DEM L289 & $05: 41: 16.687$ & $-71: 15: 05.57$ & 1 & 4.40 & 35.86 \\
\hline MCELS-L362 & DEM L292 & $05: 41: 25.704$ & $-71: 17: 00.08$ & 1 & 4.08 & 35.70 \\
\hline MCELS-L363 & DEM L285 & $05: 41: 28.354$ & $-69: 46: 36.24$ & 0 & 4.47 & 37.05 \\
\hline MCELS-L364 & DEM L295 & $05: 41: 34.522$ & $-70: 01: 19.19$ & 0 & 4.16 & 36.35 \\
\hline MCELS-L365 & DEM L294 & 05:41:35.101 & $-70: 35: 13.46$ & 3 & 5.14 & 36.64 \\
\hline MCELS-L366 & DEM L286 & $05: 41: 35.760$ & $-66: 59: 15.86$ & 0 & 0.81 & 36.26 \\
\hline MCELS-L367 & DEM L293 & $05: 41: 38.076$ & $-71: 19: 49.01$ & 2 & 4.13 & 37.78 \\
\hline MCELS-L368 & DEM L297 & $05: 42: 10.044$ & $-68: 58: 16.65$ & 0 & 5.58 & 36.57 \\
\hline MCELS-L369 & DEM L298 & $05: 42: 17.177$ & $-69: 05: 44.53$ & 1 & 5.00 & 37.70 \\
\hline MCELS-L370 & DEM L296 & $05: 42: 25.863$ & $-66: 39: 49.95$ & 0 & 0.89 & 36.55 \\
\hline MCELS-L371 & DEM L299 & $05: 42: 55.337$ & $-68: 56: 53.17$ & 4 & 5.10 & 37.30 \\
\hline MCELS-L372 & DEM L300 & 05:43:04.617 & $-69: 45: 57.35$ & 1 & 5.35 & 37.48 \\
\hline MCELS-L373 & DEM L301 & $05: 43: 17.469$ & $-67: 50: 48.26$ & 2 & 1.77 & 37.84 \\
\hline MCELS-L374 & DEM L304 & $05: 43: 46.655$ & $-67: 27: 12.21$ & 1 & 2.73 & 36.35 \\
\hline MCELS-L375 & DEM L306 & $05: 44: 22.206$ & $-67: 27: 34.70$ & 0 & 1.22 & 37.71 \\
\hline MCELS-L376 & N73 & $05: 44: 22.206$ & $-67: 27: 34.70$ & 0 & 2.48 & 35.83 \\
\hline MCELS-L377 & DEM L307 & $05: 44: 24.241$ & $-69: 22: 43.41$ & 1 & 4.80 & 36.78 \\
\hline MCELS-L378 & DEM L310 & $05: 44: 55.496$ & $-69: 27: 44.48$ & 0 & 4.49 & $38.53^{\mathrm{d}}$ \\
\hline MCELS-L379 & DEM L309-1 & $05: 45: 08.921$ & $-67: 08: 53.16$ & 2 & 1.20 & 37.57 \\
\hline MCELS-L380 & DEM L311 & $05: 45: 22.227$ & $-69: 46: 20.92$ & 1 & 4.01 & 37.04 \\
\hline MCELS-L381 & DEM L308 & $05: 45: 24.969$ & $-67: 17: 43.62$ & 0 & 1.20 & 37.79 \\
\hline MCELS-L382 & DEM L309-2 & $05: 45: 43.022$ & $-67: 09: 48.64$ & 1 & 1.67 & 36.50 \\
\hline MCELS-L383 & DEM L312 & 05:46:05.751 & $-69: 33: 28.05$ & 0 & 5.34 & 36.84 \\
\hline MCELS-L384 & DEM L313 & $05: 46: 27.250$ & $-69: 35: 21.64$ & 1 & 5.39 & 35.86 \\
\hline MCELS-L385 & DEM L314 & $05: 46: 33.871$ & $-69: 34: 21.85$ & 1 & 5.27 & 36.28 \\
\hline MCELS-L386 & DEM L315 & $05: 46: 38.797$ & $-67: 10: 38.82$ & 3 & 0.86 & 37.40 \\
\hline MCELS-L387 & DEM L317 & $05: 47: 03.980$ & $-70: 09: 03.86$ & 0 & 3.99 & 36.56 \\
\hline MCELS-L388 & DEM L316 & $05: 47: 06.273$ & $-69: 42: 32.80$ & 4 & 6.24 & 36.85 \\
\hline MCELS-L389 & DEM L318 & $05: 47: 56.636$ & $-69: 52: 10.09$ & 1 & 3.78 & 35.44 \\
\hline MCELS-L390 & DEM L320 & $05: 48: 01.857$ & $-69: 53: 51.39$ & 1 & 3.69 & 36.32 \\
\hline
\end{tabular}


Table 5

(Continued)

\begin{tabular}{|c|c|c|c|c|c|c|}
\hline Object ID & Other ID & $\begin{array}{l}\text { R.A. (J2000) } \\
\text { (h:m:s) }\end{array}$ & $\begin{array}{l}\text { Decl. } \\
\text { (d:m:s) }\end{array}$ & Type $^{\mathrm{b}}$ & $\begin{array}{c}N(\mathrm{HI}) \\
\left(10^{21} \mathrm{~cm}^{-2}\right)\end{array}$ & $\begin{array}{c}L \\
\left(\operatorname{erg~s}^{-1}\right)\end{array}$ \\
\hline MCELS-L391 & DEM L319 & 05:48:02.934 & $-69: 53: 02.42$ & 1 & 3.76 & 35.63 \\
\hline MCELS-L392 & DEM L321 & 05:48:11.322 & $-69: 52: 44.40$ & 1 & 3.83 & 35.35 \\
\hline MCELS-L393 & DEM L322 & 05:48:15.072 & $-70: 02: 02.97$ & 1 & 4.19 & 36.78 \\
\hline MCELS-L394 & DEM L324 & $05: 48: 44.604$ & $-69: 50: 37.97$ & 1 & 2.91 & 35.91 \\
\hline MCELS-L395 & DEM L325 & $05: 48: 58.024$ & $-69: 59: 42.99$ & 1 & 3.72 & 36.59 \\
\hline MCELS-L396 & DEM L323+326 & $05: 49: 23.980$ & $-70: 06: 20.17$ & 2 & 3.03 & 38.17 \\
\hline MCELS-L397 & DEM L327 & $05: 49: 27.344$ & $-69: 19: 34.15$ & 0 & 2.84 & 36.84 \\
\hline MCELS-L398 & DEM L328 & $05: 51: 29.273$ & $-68: 13: 15.29$ & 0 & 1.88 & 37.60 \\
\hline MCELS-L399 & DEM L329 & $05: 51: 41.935$ & $-69: 55: 49.08$ & 0 & 1.43 & 35.40 \\
\hline MCELS-L400 & $\mathrm{N} 75 \mathrm{~A}$ & $05: 55: 42.166$ & $-68: 09: 46.94$ & 1 & 2.08 & 35.76 \\
\hline MCELS-L401 & $\ldots$ & 05:55:54.448 & $-68: 13: 53.91$ & 1 & 1.47 & 37.43 \\
\hline
\end{tabular}

Notes.

${ }^{a}$ Identifiers in Column 2 are from Davies et al. (1976; DEM), Bica et al. (1999; BSDL), or Henize (1956; N).

${ }^{\mathrm{b}}$ Optical depth classifications in Column 5 are 0 -indeterminate, 1 -optically thick, 2-blister, 3—optically thin, and 4-shocked nebulae.

${ }^{\mathrm{c}}$ Local $\mathrm{H} \alpha$ backgrounds could not be unambiguously determined for $L$ measurements of these objects due to a high DIG luminosity, even though structure is seen in $[\mathrm{S} \mathrm{II}] /[\mathrm{O}$ III] . Therefore, the background was set to the surface brightness of the outermost area of the LMC observed by the MCELS survey, as discussed in the text.

${ }^{\mathrm{d}}$ Object includes separately cataloged substructure in the line of sight. Photometry for the substructure is not included in the photometry of the larger region.

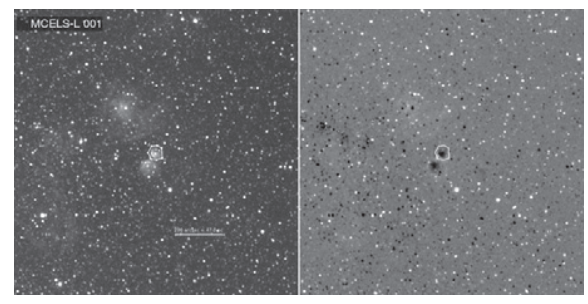

(a) MCELS-L001

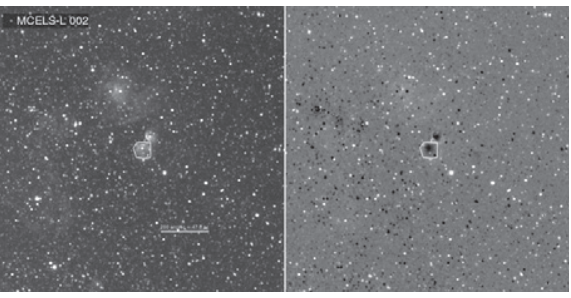

(b) MCELS-L002

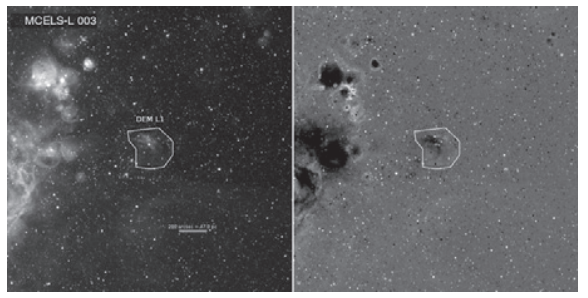

(c) MCELS-L003

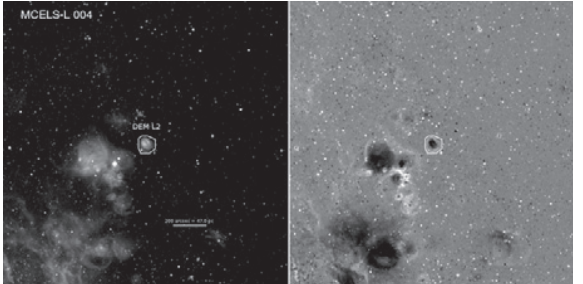

(d) MCELS-L004

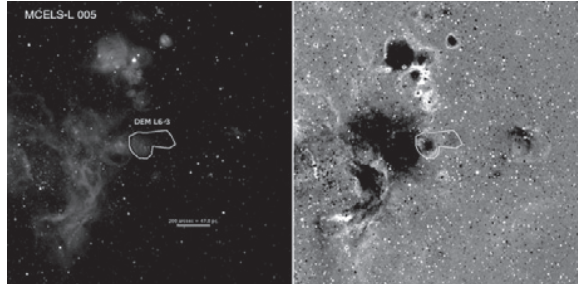

(e) MCELS-L005

Figure 23. Five LMC H II regions from the MCELS catalog in $\mathrm{H} \alpha$ (left) and [S II]/[O III] (right).

(The complete figure set (399 images) is available in the online journal)

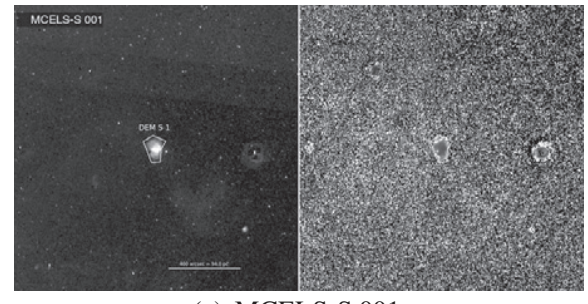

(a) MCELS-S 001

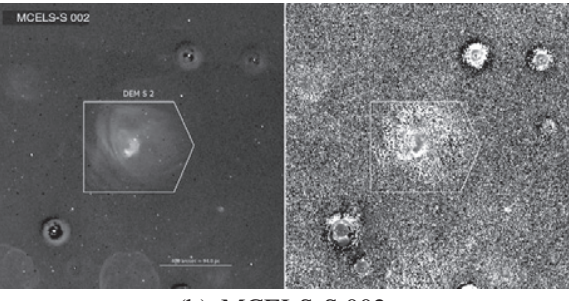

(b) MCELS-S 002

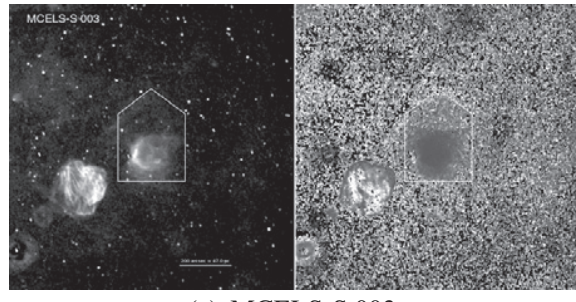

(c) MCELS-S 003

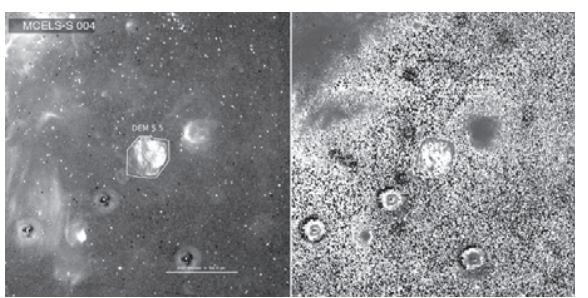

(d) MCELS-S 004

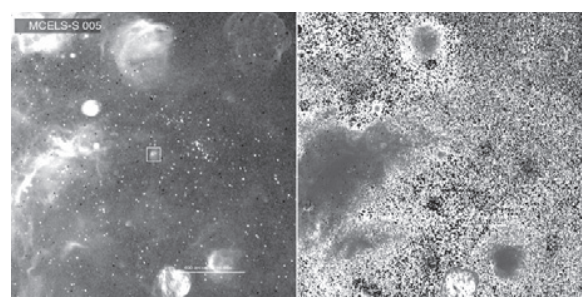

(e) MCELS-S 005

Figure 24. Five SMC H II regions from the MCELS-S catalog in $\mathrm{H} \alpha$ and $[\mathrm{S} \mathrm{II}] /[\mathrm{O}$ III].

(The complete figure set (214 images) is available in the online journal) 
Table 6

MCELS SMC H II Region Catalog

\begin{tabular}{|c|c|c|c|c|c|c|}
\hline Object ID & Other ID ${ }^{\mathrm{a}}$ & $\begin{array}{l}\text { R.A. (J2000) } \\
\text { (h:m:s) }\end{array}$ & $\begin{array}{c}\text { Decl. } \\
\text { (d:m:s) }\end{array}$ & Type $^{b}$ & $\begin{array}{c}N(\mathrm{H} \mathrm{I}) \\
\left(10^{21} \mathrm{~cm}^{-2}\right)\end{array}$ & $\begin{array}{c}L(\mathrm{H} \alpha) \\
\left(\mathrm{erg} \mathrm{s}^{-1}\right)\end{array}$ \\
\hline MCELS-S1 & DEM S1 & $00: 31: 41.06$ & $-73: 47: 38.69$ & 3 & 2.38 & 36.30 \\
\hline MCELS-S2 & DEM S2 & $00: 36: 59.04$ & $-72: 59: 41.53$ & 1 & 2.66 & 36.67 \\
\hline MCELS-S3 & $\ldots$ & $00: 39: 59.55$ & $-73: 33: 30.31$ & 1 & 2.88 & 35.88 \\
\hline MCELS-S4 & DEM S5 & $00: 41: 02.56$ & $-73: 36: 20.16$ & 1 & 3.88 & 36.21 \\
\hline MCELS-S5 & $\ldots$ & $00: 41: 39.6$ & $-73: 24: 26.18$ & 2 & 4.94 & 34.76 \\
\hline MCELS-S6 & $\ldots$ & $00: 42: 10.16$ & $-73: 14: 52.26$ & 2 & 5.77 & 36.34 \\
\hline MCELS-S7 & DEM S6 & $00: 42: 15.49$ & $-72: 59: 37.56$ & 2 & 5.36 & 35.84 \\
\hline MCELS-S8 & DEM S7 & $00: 42: 27.33$ & $-73: 43: 55.36$ & 1 & 3.11 & 35.95 \\
\hline MCELS-S9 & $\ldots$ & $00: 42: 55.25$ & $-74: 28: 51.55$ & 1 & 1.93 & 36.11 \\
\hline MCELS-S10 & $\ldots$ & 00:43:06.71 & $-73: 20: 30.35$ & 2 & 6.82 & 35.85 \\
\hline MCELS-S11 & DEM S8 & 00:43:08 & $-72: 35: 53.66$ & 2 & 1.86 & 34.54 \\
\hline MCELS-S12 & $\ldots$ & $00: 43: 18.68$ & $-73: 13: 52.54$ & 3 & 6.48 & 34.57 \\
\hline MCELS-S13 & $\ldots$ & $00: 43: 34.91$ & $-73: 15: 44.58$ & 1 & 7.63 & 34.67 \\
\hline MCELS-S14 & DEM S9 & $00: 43: 35.76$ & $-73: 02: 27.75$ & 2 & 5.36 & 36.34 \\
\hline MCELS-S15 & $\ldots$ & $00: 43: 44.93$ & $-73: 08: 52.52$ & 1 & 6.30 & 36.70 \\
\hline MCELS-S16 & $\ldots$ & $00: 43: 47.31$ & $-73: 15: 55.19$ & 2 & 8.11 & 34.34 \\
\hline MCELS-S17 & DEM S10 & $00: 43: 50.78$ & $-73: 28: 29.27$ & 1 & 6.12 & 36.96 \\
\hline MCELS-S18 & $\ldots$ & $00: 44: 54.2$ & $-72: 55: 59.57$ & 1 & 4.13 & 35.07 \\
\hline MCELS-S19 & N10 & $00: 44: 54.42$ & $-73: 10: 23.89$ & 1 & 8.43 & 36.13 \\
\hline MCELS-S20 & DEM S15+18 & $00: 45: 20.59$ & $-73: 03: 39.39$ & 1 & 8.60 & 37.44 \\
\hline MCELS-S21 & DEM S12 & $00: 45: 21.04$ & $-73: 59: 13.4$ & 3 & 2.89 & 36.29 \\
\hline MCELS-S22 & N13B & $00: 45: 21.38$ & $-73: 22: 27.5$ & 1 & 8.82 & 36.71 \\
\hline MCELS-S23 & N13 & $00: 45: 24.44$ & $-73: 23: 05.56$ & 1 & 8.43 & 36.65 \\
\hline MCELS-S24 & DEM S14+19 & $00: 45: 25.89$ & $-73: 15: 12.29$ & 1 & 9.54 & 37.11 \\
\hline MCELS-S25 & DEM S17 & $00: 45: 32.31$ & $-73: 12: 29.89$ & 1 & 9.16 & 36.48 \\
\hline MCELS-S26 & DEM S20 & 00:46:09.56 & $-73: 05: 59.47$ & 1 & 10.01 & 37.16 \\
\hline MCELS-S27 & N15 & 00:46:11.04 & $-73: 25: 37.07$ & 1 & 7.43 & 35.67 \\
\hline MCELS-S28 & N21 & $00: 46: 19.81$ & $-73: 23: 32.9$ & 1 & 8.35 & 36.59 \\
\hline MCELS-S29 & DEM S29 & $00: 46: 24.28$ & $-73: 26: 21.9$ & 1 & 7.80 & $37.61^{\mathrm{e}}$ \\
\hline MCELS-S30 & DEM S22 & $00: 46: 24.84$ & $-73: 12: 27.63$ & 1 & 8.78 & 36.55 \\
\hline MCELS-S31 & DEM S23 & $00: 46: 31.53$ & $-73: 06: 16.48$ & 1 & 10.86 & 37.40 \\
\hline MCELS-S32 & $\ldots$ & $00: 46: 38.48$ & $-72: 54: 39.88$ & 1 & 6.61 & 34.89 \\
\hline MCELS-S33 & DEM S24 & 00:46:40.99 & $-73: 21: 36.61$ & 1 & 9.64 & 36.61 \\
\hline MCELS-S34 & DEM S25 & $00: 46: 42.99$ & $-73: 31: 47.77$ & 1 & 6.44 & 37.14 \\
\hline MCELS-S35 & $\ldots$ & $00: 47: 00.86$ & $-73: 18: 04.12$ & 1 & 10.16 & 36.76 \\
\hline MCELS-S36 & $\ldots$ & $00: 47: 07.48$ & $-73: 14: 11.46$ & 3 & 9.46 & 36.24 \\
\hline MCELS-S37 & DEM S31 & $00: 47: 29.07$ & $-73: 05: 08.14$ & 2 & 13.93 & 36.82 \\
\hline MCELS-S38 & DEM S30 & $00: 47: 30.32$ & $-73: 22: 17.26$ & 1 & 8.90 & 36.66 \\
\hline MCELS-S39 & DEM S32 & $00: 47: 43.89$ & $-73: 08: 22.08$ & 1 & 11.73 & 37.74 \\
\hline MCELS-S40 & DEM S35 & $00: 47: 46.41$ & $-73: 17: 30.87$ & 1 & 10.46 & 36.26 \\
\hline MCELS-S41 & DEM S34 & $00: 47: 53.71$ & $-73: 17: 35.64$ & 2 & 10.00 & 34.89 \\
\hline MCELS-S42 & DEM S36 & $00: 47: 57.44$ & $-73: 17: 38.04$ & 1 & 9.96 & 36.48 \\
\hline MCELS-S43 & DEM S33 & $00: 48: 03.27$ & $-73: 35: 11.86$ & 1 & 4.94 & 36.14 \\
\hline MCELS-S44 & DEM S37 & 00:48:03.91 & $-73: 16: 22.84$ & 1 & 9.98 & 37.33 \\
\hline MCELS-S45 & DEM S38 & 00:48:09.13 & $-73: 14: 09.48$ & 1 & 10.73 & 36.80 \\
\hline MCELS-S46 & $\ldots$ & $00: 48: 15.77$ & $-73: 11: 16.7$ & 2 & 10.61 & 35.57 \\
\hline MCELS-S47 & DEM S39 & $00: 48: 18.08$ & $-73: 10: 18.59$ & 1 & 10.98 & 35.8 \\
\hline MCELS-S48 & DEM S42 & $00: 48: 18.88$ & $-73: 19: 42.55$ & 1 & 10.02 & 36.84 \\
\hline MCELS-S49 & DEM S41 & $00: 48: 21.63$ & $-73: 32: 52.29$ & 1 & 4.93 & 36.68 \\
\hline MCELS-S50 & N27 & $00: 48: 24$ & $-73: 05: 49.63$ & 0 & 13.27 & 36.41 \\
\hline MCELS-S51 & DEM S43 & $00: 48: 26.68$ & $-73: 15: 16.09$ & 1 & 9.78 & 37.12 \\
\hline MCELS-S52 & $\ldots$ & $00: 48: 28.3$ & $-72: 15: 58.18$ & 1 & 2.87 & 36.73 \\
\hline MCELS-S53 & $\ldots$ & $00: 48: 32.64$ & $-72: 52: 57.92$ & 2 & 9.57 & 35.78 \\
\hline MCELS-S54 & DEM S51 & $00: 48: 56.28$ & $-73: 03: 55.09$ & 1 & 10.80 & $37.51^{\mathrm{e}}$ \\
\hline MCELS-S55 & $\ldots$ & $00: 48: 56.6$ & $-73: 11: 40.24$ & 1 & 10.64 & 35.59 \\
\hline MCELS-S56 & DEM S45 & 00:49:01.82 & $-73: 08: 24.13$ & 1 & 12.06 & 37.43 \\
\hline MCELS-S57 & DEM S44 & $00: 49: 12.03$ & $-73: 28: 18.81$ & 1 & 6.72 & $37.26^{\mathrm{e}}$ \\
\hline MCELS-S58 & DEM S47 & $00: 49: 14.61$ & $-72: 52: 44.96$ & 1 & 9.83 & 36.90 \\
\hline MCELS-S59 & DEM S46e & $00: 49: 29.35$ & $-72: 47: 44.25$ & 1 & 7.01 & $36.45^{\mathrm{e}}$ \\
\hline MCELS-S60 & DEM S49 & $00: 49: 36.45$ & $-73: 15: 42.73$ & 1 & 8.15 & 36.77 \\
\hline MCELS-S61 & $\ldots$ & $00: 49: 37.26$ & $-73: 25: 29.72$ & 2 & 7.20 & 35.47 \\
\hline MCELS-S62 & N32 & $00: 49: 39.38$ & $-72: 48: 47.47$ & 2 & 8.57 & 36.26 \\
\hline MCELS-S63 & DEM S50 & $00: 49: 45.04$ & $-73: 10: 30.89$ & 2 & 9.64 & 35.81 \\
\hline MCELS-S64 & DEM S47-1 & 00:49:47.01 & $-72: 56: 33.6$ & 1 & 10.64 & 36.80 \\
\hline MCELS-S65 & $\ldots$ & $00: 49: 52.31$ & $-73: 24: 10.59$ & 3 & 7.66 & 36.08 \\
\hline
\end{tabular}


Table 6

(Continued)

\begin{tabular}{|c|c|c|c|c|c|c|}
\hline Object ID & Other ID ${ }^{\mathrm{a}}$ & $\begin{array}{l}\text { R.A. (J2000) } \\
\text { (h:m:s) }\end{array}$ & $\begin{array}{l}\text { Decl. } \\
\text { (d:m:s) }\end{array}$ & Type $^{b}$ & $\begin{array}{c}N(\mathrm{H} \mathrm{I}) \\
\left(10^{21} \mathrm{~cm}^{-2}\right)\end{array}$ & $\begin{array}{c}L(\mathrm{H} \alpha) \\
\left(\mathrm{erg} \mathrm{s}^{-1}\right)\end{array}$ \\
\hline MCELS-S66 & & 00:49:52.68 & $-73: 25: 38.95$ & 2 & 7.69 & 36.51 \\
\hline MCELS-S67 & DEM S46+55 & $00: 49: 57.24$ & $-72: 44: 56.91$ & 2 & 4.94 & 37.95 \\
\hline MCELS-S68 & DEM S57 & 00:50:00.64 & $-72: 32: 43.39$ & 1 & 4.20 & 36.43 \\
\hline MCELS-S69 & DEM S56 & $00: 50: 25.69$ & $-72: 35: 36.05$ & 4 & 3.86 & 37.21 \\
\hline MCELS-S70 & $\ldots$ & $00: 50: 30.17$ & $-73: 31: 35.55$ & 1 & 6.74 & 36.57 \\
\hline MCELS-S71 & DEM S54 & $00: 50: 33.85$ & $-72: 53: 26.04$ & 1 & 10.46 & $37.84^{\mathrm{e}}$ \\
\hline MCELS-S72 & DEM S52 & $00: 50: 34.6$ & $-73: 20: 11.25$ & 1 & 8.04 & 36.49 \\
\hline MCELS-S73 & DEM S53 & $00: 50: 48.75$ & $-73: 24: 22.31$ & 3 & 7.20 & 35.76 \\
\hline MCELS-S74 & N41 & 00:51:01.66 & $-72: 52: 52.46$ & 1 & 9.91 & 36.24 \\
\hline MCELS-S75 & $\ldots$ & $00: 51: 06.31$ & $-73: 31: 35.57$ & 0 & 6.87 & 35.04 \\
\hline MCELS-S76 & $\ldots$ & $00: 51: 14.14$ & $-73: 31: 35.97$ & 1 & 6.91 & 34.92 \\
\hline MCELS-S77 & DEM S59 & 00:51:19.13 & $-73: 30: 15.17$ & 1 & 6.71 & 36.54 \\
\hline MCELS-S78 & DEM S63 & $00: 51: 34.74$ & $-72: 41: 21.88$ & 1 & 5.39 & 37.14 \\
\hline MCELS-S79 & H86-117 & $00: 51: 40.63$ & $-73: 31: 50.82$ & 1 & 7.56 & 34.62 \\
\hline MCELS-S80 & DEM S62 & 00:51:47.79 & $-72: 50: 46.86$ & 1 & 9.58 & 36.15 \\
\hline MCELS-S81 & DEM S65 & $00: 51: 58.26$ & $-72: 16: 30.62$ & 1 & 5.18 & 36.70 \\
\hline MCELS-S82 & DEM S60 & 00:52:01.65 & $-73: 13: 18.95$ & 3 & 8.80 & 36.64 \\
\hline MCELS-S83 & & $00: 52: 04.85$ & $-72: 44: 57.81$ & 1 & 6.72 & 37.07 \\
\hline MCELS-S84 & DEM S63 & $00: 52: 05.56$ & $-72: 39: 35.94$ & 1 & 7.02 & 36.68 \\
\hline MCELS-S85 & DEM S67 & $00: 52: 15.11$ & $-71: 50: 18.01$ & 1 & 1.50 & 36.56 \\
\hline MCELS-S86 & DEM S71 & $00: 52: 18.21$ & $-73: 27: 06.57$ & 1 & 8.16 & 35.92 \\
\hline MCELS-S87 & DEM S66 & $00: 52: 25.42$ & $-72: 08: 59.18$ & 1 & 2.82 & $37.50^{\mathrm{e}}$ \\
\hline MCELS-S88 & DEM S72 & $00: 52: 37.19$ & $-73: 26: 11.11$ & 1 & 8.21 & 36.21 \\
\hline MCELS-S89 & $\ldots$ & 00:52:39.49 & $-72: 55: 29.96$ & 1 & 7.05 & 35.17 \\
\hline MCELS-S90 & DEM S69 & $00: 53: 01.5$ & $-72: 53: 42.46$ & 1 & 6.32 & 36.62 \\
\hline MCELS-S91 & DEM S74 & $00: 53: 23.64$ & $-73: 12: 07.57$ & 1 & 9.13 & 36.96 \\
\hline MCELS-S92 & $\ldots$ & $00: 53: 25.53$ & $-72: 28: 30.4$ & 1 & 4.79 & 35.36 \\
\hline MCELS-S93 & N52-2 & $00: 53: 40.64$ & $-72: 39: 37.59$ & 2 & 7.52 & 35.82 \\
\hline MCELS-S94 & N52-1 & 00:53:43.06 & $-72: 39: 20.91$ & 2 & 7.48 & 35.82 \\
\hline MCELS-S95 & DEM S76 & 00:54:01.46 & $-72: 22: 23.27$ & 1 & 3.54 & 36.60 \\
\hline MCELS-S96 & DEM S78 & $00: 54: 11.18$ & $-73: 17: 17.42$ & 3 & 8.34 & 36.19 \\
\hline MCELS-S97 & $\ldots$ & $00: 54: 15.65$ & $-73: 32: 16.91$ & 1 & 5.56 & 35.49 \\
\hline MCELS-S98 & DEM S80 & $00: 54: 22.97$ & $-72: 42: 49.02$ & 2 & 7.10 & 37.78 \\
\hline MCELS-S99 & DEM S79 & $00: 54: 29.15$ & $-71: 57: 57.73$ & 3 & 1.86 & 36.02 \\
\hline MCELS-S100 & $\ldots$ & 00:54:56.91 & $-73: 19: 14.26$ & 4 & 5.57 & 35.04 \\
\hline MCELS-S101 & $\ldots$ & 00:55:02.43 & $-72: 55: 59.24$ & 1 & 6.37 & 35.94 \\
\hline MCELS-S102 & $\ldots$ & $00: 55: 14.71$ & $-72: 26: 32.68$ & 1 & 5.58 & 35.65 \\
\hline MCELS-S103 & $\ldots$ & $00: 55: 34.38$ & $-72: 29: 12.19$ & 1 & 5.30 & 35.37 \\
\hline MCELS-S104 & DEM S83NE & $00: 55: 34.39$ & $-72: 17: 12.14$ & 3 & 5.63 & 35.94 \\
\hline MCELS-S105 & DEM S84-1 & $00: 55: 44.14$ & $-72: 16: 02.02$ & 2 & 5.71 & 35.87 \\
\hline MCELS-S106 & $\ldots$ & 00:55:48.91 & $-72: 38: 14.17$ & 1 & 7.61 & 35.39 \\
\hline MCELS-S107 & DEM S81 & $00: 55: 57.38$ & $-73: 23: 16.6$ & 1 & 3.87 & 36.86 \\
\hline MCELS-S108 & DEM S84-2 & $00: 56: 02.19$ & $-72: 15: 43.54$ & 2 & 6.42 & 35.66 \\
\hline MCELS-S109 & $\ldots$ & $00: 56: 03.53$ & $-72: 27: 11.41$ & 3 & 5.63 & 36.11 \\
\hline MCELS-S110 & DEM S85 & $00: 56: 16.35$ & $-72: 17: 25.12$ & 1 & 6.59 & 36.56 \\
\hline MCELS-S111 & DEM S88 & $00: 56: 18.55$ & $-72: 47: 24.49$ & 3 & 8.53 & 36.13 \\
\hline MCELS-S112 & DEM S90 & $00: 56: 46.91$ & $-72: 03: 21.81$ & 1 & 3.23 & 36.70 \\
\hline MCELS-S113 & DEM S89 & $00: 56: 48.11$ & $-72: 47: 46.96$ & 2 & 9.21 & 35.47 \\
\hline MCELS-S114 & $\ldots$ & 00:57:02.52 & $-72: 21: 50.87$ & 2 & 7.22 & 35.80 \\
\hline MCELS-S115 & DEM S91 & $00: 57: 10.34$ & $-73: 34: 17.33$ & 1 & 3.06 & 36.19 \\
\hline MCELS-S116 & $\ldots$ & $00: 57: 18.3$ & $-71: 54: 38.54$ & 1 & 3.28 & 35.31 \\
\hline MCELS-S117 & $\ldots$ & $00: 57: 38.4$ & $-72: 24: 40.33$ & 1 & 8.04 & 35.42 \\
\hline MCELS-S118 & DEM S93 & $00: 57: 56.71$ & $-72: 39: 20.64$ & 0 & 7.86 & 36.47 \\
\hline MCELS-S119 & DEM S94 & $00: 58: 16.58$ & $-72: 38: 53.47$ & 1 & 7.75 & 36.57 \\
\hline MCELS-S120 & SNR B0056-72.5 & $00: 58: 17.01$ & $-72: 17: 52.65$ & 4 & 5.66 & 36.45 \\
\hline MCELS-S121 & DEM S95 & $00: 58: 20.07$ & $-72: 40: 09.75$ & 1 & 7.50 & 36.68 \\
\hline MCELS-S122 & DEM S96 & $00: 58: 28.05$ & $-71: 44: 39.84$ & 1 & 3.18 & 36.11 \\
\hline MCELS-S123 & DEM S97 & $00: 58: 30.59$ & $-71: 31: 07.05$ & 1 & 1.84 & $36.66^{\mathrm{e}}$ \\
\hline MCELS-S124 & DEM S98 & $00: 58: 36.63$ & $-72: 14: 08.1$ & 3 & 6.80 & 36.37 \\
\hline MCELS-S125 & DEM S99 & $00: 58: 57.33$ & $-72: 14: 36.03$ & 3 & 5.85 & 36.18 \\
\hline MCELS-S126 & $\ldots$ & 00:59:05.94 & $-71: 45: 18.56$ & 2 & 3.66 & 34.51 \\
\hline MCELS-S127 & DEM S100 & 00:59:14.98 & $-72: 24: 15.09$ & 3 & 7.43 & 36.18 \\
\hline MCELS-S128 & DEM S102 & 00:59:18.75 & $-72: 17: 31.29$ & 1 & 4.69 & 36.91 \\
\hline MCELS-S129 & DEM S105 & 00:59:42.11 & $-71: 43: 43.27$ & 0 & 3.86 & 36.44 \\
\hline MCELS-S130 & N66,NGC 346 & $00: 59: 42.58$ & $-72: 12: 05.08$ & 1 & 3.91 & 38.81 \\
\hline
\end{tabular}


Table 6

(Continued)

\begin{tabular}{|c|c|c|c|c|c|c|}
\hline Object ID & Other ID ${ }^{\mathrm{a}}$ & $\begin{array}{l}\text { R.A. (J2000) } \\
\text { (h:m:s) }\end{array}$ & $\begin{array}{l}\text { Decl. } \\
\text { (d:m:s) }\end{array}$ & Type $^{\text {b }}$ & $\begin{array}{c}N(\mathrm{H} \mathrm{I}) \\
\left(10^{21} \mathrm{~cm}^{-2}\right)\end{array}$ & $\begin{array}{c}L(\mathrm{H} \alpha) \\
\left(\mathrm{erg} \mathrm{s}^{-1}\right)\end{array}$ \\
\hline MCELS-S131 & DEM S107 & 01:00:15.24 & $-71: 48: 25.27$ & 2 & 4.47 & 36.28 \\
\hline MCELS-S132 & DEM S109 & 01:00:58.56 & $-71: 35: 27.31$ & 3 & 4.22 & 36.11 \\
\hline MCELS-S133 & DEM S108 & 01:01:17.67 & $-71: 30: 59.48$ & 1 & 3.31 & 37.28 \\
\hline MCELS-S134 & DEM S113 & 01:01:30.27 & $-71: 47: 45.25$ & 2 & 5.00 & 35.64 \\
\hline MCELS-S135 & DEM S112 & 01:01:30.73 & $-71: 51: 07.81$ & 1 & 4.25 & 36.47 \\
\hline MCELS-S136 & DEM S111 & 01:01:43.19 & $-71: 56: 16.37$ & 0 & 4.53 & 36.42 \\
\hline MCELS-S137 & $\ldots$ & 01:01:53.64 & $-72: 06: 09.74$ & 1 & 4.37 & 36.67 \\
\hline MCELS-S138 & $\ldots$ & 01:01:59.85 & $-71: 54: 54.31$ & 2 & 5.41 & 35.63 \\
\hline MCELS-S139 & DEM S115 & 01:02:16.57 & $-71: 51: 25.29$ & 1 & 5.05 & 36.23 \\
\hline MCELS-S140 & DEM S116 & 01:02:28.61 & $-71: 56: 33.64$ & 2 & 5.62 & 35.41 \\
\hline MCELS-S141 & DEM S118 & 01:02:41.09 & $-72: 24: 40.93$ & 1 & 5.22 & 36.74 \\
\hline MCELS-S142 & DEM S117b-1 & 01:02:43.45 & $-71: 53: 34.15$ & 1 & 5.69 & 35.56 \\
\hline MCELS-S143 & DEM S117 & 01:02:47.82 & $-71: 53: 18.21$ & 3 & 5.71 & 36.41 \\
\hline MCELS-S144 & DEM S119+120 & 01:03:01.24 & $-72: 05: 40.88$ & 1 & 6.24 & 36.49 \\
\hline MCELS-S145 & DEM S121 & 01:03:03.09 & $-71: 53: 29.98$ & 3 & 5.54 & 36.29 \\
\hline MCELS-S146 & SNR B0101-72.6 & 01:03:17.4 & $-72: 09: 43.17$ & 1 & 5.75 & 35.29 \\
\hline MCELS-S147 & DEM S123 & 01:03:25.01 & $-72: 03: 44.83$ & 1 & 6.04 & 37.90 \\
\hline MCELS-S148 & $\mathrm{N} 76 \mathrm{~A}$ & 01:03:48.56 & $-72: 03: 56.12$ & 3 & 5.99 & 36.18 \\
\hline MCELS-S149 & DEM S122 & 01:03:58.67 & $-72: 40: 53.09$ & 1 & 5.16 & 35.86 \\
\hline MCELS-S150 & DEM S124 & 01:04:08.35 & $-72: 02: 07.4$ & 1 & 5.62 & 36.51 \\
\hline MCELS-S151 & DEM S125 & 01:04:14.98 & $-72: 23: 59.99$ & 2 & 4.76 & 34.72 \\
\hline MCELS-S152 & $\ldots$ & 01:04:22.01 & $-71: 56: 45.55$ & 2 & 5.73 & 35.77 \\
\hline MCELS-S153 & N78 & 01:05:03.14 & $-71: 59: 21.94$ & 2 & 6.52 & 36.35 \\
\hline MCELS-S154 & $\mathrm{N} 78 \mathrm{~A}$ & 01:05:04.38 & $-71: 58: 57.96$ & 3 & 6.37 & 36.03 \\
\hline MCELS-S155 & $\ldots$ & 01:05:07.69 & $-72: 12: 14.31$ & 1 & 5.14 & 37.34 \\
\hline MCELS-S156 & DEM S129 & 01:05:09.45 & $-72: 48: 04.59$ & 1 & 5.31 & 37.05 \\
\hline MCELS-S157 & DEM S127 & 01:05:12.13 & $-71: 58: 25.05$ & 2 & 6.28 & 36.41 \\
\hline MCELS-S158 & DEM S126 & 01:05:12.8 & $-72: 00: 37.7$ & 1 & 6.00 & 37.51 \\
\hline MCELS-S159 & DEM S128 & 01:05:23.52 & $-72: 08: 42.38$ & 3 & 5.19 & 36.29 \\
\hline MCELS-S160 & DEM S130 & 01:05:41.25 & $-72: 03: 48.38$ & 3 & 5.96 & 36.25 \\
\hline MCELS-S161 & DEM S134 & 01:05:52.12 & $-72: 29: 56.1$ & 1 & 4.60 & 37.21 \\
\hline MCELS-S162 & $\ldots$ & 01:05:55.46 & $-72: 19: 47.13$ & 2 & 4.51 & 35.16 \\
\hline MCELS-S163 & DEM S131 & 01:06:18.09 & $-72: 05: 23.61$ & 4 & 5.96 & 36.53 \\
\hline MCELS-S164 & DEM S132 & 01:06:24.96 & $-71: 57: 37.47$ & 1 & 6.56 & 37.21 \\
\hline MCELS-S165 & $\ldots$ & 01:06:41.51 & $-73: 10: 02$ & 2 & 5.76 & 35.67 \\
\hline MCELS-S166 & DEM S134 & 01:06:56.07 & $-72: 33: 06.11$ & 1 & 5.87 & $36.69^{\mathrm{e}}$ \\
\hline MCELS-S167 & $\ldots$ & 01:07:27.03 & $-73: 33: 13.15$ & 1 & 2.84 & 35.65 \\
\hline MCELS-S168 & DEM S133 & 01:07:34.66 & $-72: 51: 19.68$ & 1 & 5.32 & 36.91 \\
\hline MCELS-S169 & DEM S135 & 01:08:09.89 & $-71: 59: 49.92$ & 1 & 6.17 & 37.48 \\
\hline MCELS-S170 & DEM S136 & 01:09:04.95 & $-71: 51: 36.76$ & 1 & 4.48 & 36.58 \\
\hline MCELS-S171 & DEM S138 & 01:09:16.95 & $-73: 10: 59.22$ & 1 & 4.83 & 37.39 \\
\hline MCELS-S172 & $\ldots$ & 01:09:27.3 & $-72: 01: 28.08$ & 3 & 4.83 & 36.10 \\
\hline MCELS-S173 & $\ldots$ & 01:09:41.52 & $-73: 18: 15.73$ & 1 & 4.15 & 35.23 \\
\hline MCELS-S174 & $\ldots$ & 01:09:50.35 & $-72: 30: 49.98$ & 1 & 4.00 & 36.42 \\
\hline MCELS-S175 & $\ldots$ & 01:10:44.06 & $-72: 21: 24.9$ & 2 & 3.97 & 35.87 \\
\hline MCELS-S176 & DEM S140 & 01:10:49.69 & $-72: 43: 16.7$ & 1 & 4.64 & 37.41 \\
\hline MCELS-S177 & $\ldots$ & 01:11:05.12 & $-72: 13: 48.03$ & 3 & 4.23 & 36.03 \\
\hline MCELS-S178 & DEM S142 & 01:11:20.51 & $-72: 09: 50.47$ & 1 & 4.22 & 36.69 \\
\hline MCELS-S179 & DEM S141 & 01:11:35.37 & $-72: 21: 56.98$ & 1 & 4.03 & 37.06 \\
\hline MCELS-S180 & DEM S145 & 01:11:41.91 & $-73: 13: 25.92$ & 1 & 5.34 & 36.29 \\
\hline MCELS-S181 & & 01:11:43.48 & $-73: 17: 51.67$ & 1 & 4.46 & $36.46^{\mathrm{e}}$ \\
\hline MCELS-S182 & DEM S144 & 01:11:53.48 & $-72: 44: 18.98$ & 3 & 4.95 & 36.24 \\
\hline MCELS-S183 & DEM S143 & 01:12:14.01 & $-72: 15: 28.02$ & 2 & 4.18 & 35.80 \\
\hline MCELS-S184 & DEM S147 & 01:13:42.09 & $-73: 17: 49.62$ & 1 & 6.16 & 37.68 \\
\hline MCELS-S185 & DEM S148 & 01:13:51.62 & $-73: 15: 45.55$ & 2 & 7.35 & 35.94 \\
\hline MCELS-S186 & DEM S147-1 & 01:14:00.32 & $-73: 17: 15.51$ & 1 & 6.82 & 36.73 \\
\hline MCELS-S187 & DEM S149 & 01:14:16.9 & $-73: 15: 53.44$ & 3 & 7.10 & 36.35 \\
\hline MCELS-S188 & DEM S150-1 & $01: 14: 22.13$ & $-73: 14: 33.85$ & 1 & 6.99 & 36.57 \\
\hline MCELS-S189 & N84 & $01: 14: 27.73$ & $-73: 12: 50.68$ & 1 & 6.80 & $36.86^{\mathrm{e}}$ \\
\hline MCELS-S190 & DEM S151-1 & $01: 14: 38.13$ & $-73: 16: 04.7$ & 1 & 6.63 & 36.53 \\
\hline MCELS-S191 & DEM S151 & 01:14:41.71 & $-73: 18: 06.48$ & 1 & 6.13 & 37.25 \\
\hline MCELS-S192 & N84b & 01:14:47.15 & $-73: 19: 47.91$ & 3 & 5.86 & 36.03 \\
\hline MCELS-S193 & DEM S152-1 & 01:14:55.69 & $-73: 20: 10.33$ & 0 & 5.78 & 36.16 \\
\hline MCELS-S194 & DEM S154 & 01:15:04.61 & $-72: 19: 33.15$ & 2 & 3.38 & 35.88 \\
\hline MCELS-S195 & DEM S152 & $01: 15: 04.7$ & $-73: 19: 10.14$ & 1 & 5.71 & 36.98 \\
\hline
\end{tabular}


Table 6

(Continued)

\begin{tabular}{|c|c|c|c|c|c|c|}
\hline Object ID & Other ID ${ }^{\mathrm{a}}$ & $\begin{array}{l}\text { R.A. (J2000) } \\
\text { (h:m:s) }\end{array}$ & $\begin{array}{l}\text { Decl. } \\
\text { (d:m:s) }\end{array}$ & Type $^{b}$ & $\begin{array}{c}N(\mathrm{H} \mathrm{I}) \\
\left(10^{21} \mathrm{~cm}^{-2}\right)\end{array}$ & $\begin{array}{c}L(\mathrm{H} \alpha) \\
\left(\mathrm{erg} \mathrm{s}^{-1}\right)\end{array}$ \\
\hline MCELS-S196 & DEM S153 & $01: 15: 12.85$ & $-72: 56: 31.6$ & 1 & 4.39 & 35.79 \\
\hline MCELS-S197 & DEM S156 & 01:16:13.58 & $-73: 10: 06.48$ & 1 & 6.47 & 36.87 \\
\hline MCELS-S198 & DEM S157 & 01:16:14.74 & $-73: 25: 42.41$ & 1 & 4.04 & $38.27^{\mathrm{e}}$ \\
\hline MCELS-S199 & DEM S158 & 01:16:49.27 & $-73: 09: 05.9$ & 2 & 6.36 & 36.17 \\
\hline MCELS-S200 & DEM S159 & 01:17:05.74 & $-73: 12: 23.86$ & 1 & 5.04 & 36.34 \\
\hline MCELS-S201 & $\ldots$ & 01:19:35.57 & $-73: 05: 48.4$ & 3 & 4.55 & 35.95 \\
\hline MCELS-S202 & N87 & 01:21:06.66 & $-73: 15: 04.13$ & 0 & 3.45 & 35.91 \\
\hline MCELS-S203 & $\ldots$ & $01: 21: 13.34$ & $-73: 06: 14.51$ & 2 & 4.20 & 36.26 \\
\hline MCELS-S204 & DEM S160 & 01:23:13.13 & $-73: 22: 28.19$ & 2 & 3.11 & 36.55 \\
\hline MCELS-S205 & DEM S160S & 01:23:39.01 & $-73: 24: 02.97$ & 2 & 2.75 & 35.56 \\
\hline MCELS-S206 & $\ldots$ & 01:24:02.43 & $-73: 17: 53.01$ & 2 & 2.87 & 35.23 \\
\hline MCELS-S207 & DEM S161n & $01: 24: 14.58$ & $-73: 09: 31.3$ & 1 & 4.64 & 36.88 \\
\hline MCELS-S208 & DEM S161 & $01: 24: 45.37$ & $-73: 09: 33.64$ & 1 & 4.18 & $37.07^{\mathrm{e}}$ \\
\hline MCELS-S209 & DEM S162 & 01:24:48.85 & $-73: 27: 33.93$ & 1 & 2.71 & 36.68 \\
\hline MCELS-S210 & DEM S163 & 01:25:03.97 & $-73: 16: 40.87$ & 1 & 2.91 & 36.93 \\
\hline MCELS-S211 & DEM S164 & 01:25:53.92 & $-73: 22: 41.48$ & 1 & 2.51 & 37.37 \\
\hline MCELS-S212 & DEM S165 & 01:27:03.28 & $-73: 08: 31.86$ & 1 & 2.92 & 36.95 \\
\hline MCELS-S213 & DEM S167 & 01:29:09.94 & $-73: 24: 49.67$ & 3 & 1.51 & 37.99 \\
\hline MCELS-S214 & DEM S166 & 01:29:26.79 & $-73: 32: 38.44$ & 1 & 2.02 & 37.54 \\
\hline
\end{tabular}

Notes.

${ }^{a}$ Identifiers in Column 2 are from Davies et al. (1976; DEM), Bica et al. (1999; BSDL), or Henize (1956; N).

${ }^{\mathrm{b}}$ Optical depth classifications listed in Column 5 are 0 —indeterminate, 1 -optically thick, 2 —blister, 3 —optically thin, and 4 -shocked nebulae.

${ }^{\mathrm{c}}$ Object identified in Ye et al. (1995).

d Object identified in Ye et al. (1991).

${ }^{\mathrm{e}}$ Object includes separately cataloged substructure in the line of sight. Photometry for the substructure is not included in the photometry of the larger region.

surface brightness. With the object-observer distance and 2D spatial resolution $\Delta x$ and $\Delta y$, observations can be simulated for any arcsecond, parsec, or centimeter pixel scale. Line strength is given in units of surface brightness, which is independent of the distance between observer and object, for resolved nebulae.

A schematic of a CLOUDY simulation is shown in Figure 22(a). The uneven spacing of shells around the ionizing star reflects the individual zones calculated within a CLOUDY simulation, which are set by changes in the physical conditions of the gas as a function of depth. The CLOUDY simulation calculates the isotropic volume emissivity $\epsilon$ of each emission line in each radial zone. At each $x, y$ coordinate projected in the plane perpendicular to the line of sight $z$, SURFBRIGHT calculates the observed surface brightness in the $i$ th emission line from all CLOudy shells according to

$$
\begin{aligned}
S_{i}= & \sum_{j} d l_{j}(x, y) \times \epsilon_{i}(x, y, z) / 4 \pi \times 2.3504 \\
& \times 10^{-11} \mathrm{erg} \mathrm{s}^{-1} \mathrm{~cm}^{-2} \operatorname{arcsec}^{-2},
\end{aligned}
$$

where $d l_{j}$ is the path length along the line of sight (z-axis) through shell $j$ at the projected position $x$, and $y$ and $\epsilon_{i}$ are the local volume emissivities taken from the 1D CLOUDY simulation where the 1D radius $r$ in the model is equal to the magnitude of the radius of the geometrically defined cloud $\mathbf{r}(x, y, z)$.

Our method of integrating the emission along the line of sight, from the far to near side of the HiI region, also allows us to include the effects of internal extinction, which is important for nebulae with a high observed column density, such as photodissociation regions and molecular clouds (Pellegrini et al. 2009). Computationally, we first determine the line flux entering a given shell, which is added to the diffuse flux in that shell. The sum is locally extinguished by the internal extinction in a shell calculated by CLOUDY scaled by $d l / d r$, where $d r$ is the shell thickness. This determines the flux entering the next shell along a line of sight toward the observer.

SURFBRIGHT currently includes generic geometrical configurations for planar slabs and simple spheres. The orientation, length, and inclination of the slab are free parameters. Whole and truncated spheres as in Figure 22(b) are also possible. Future support will include completely arbitrary geometric configurations. Currently, the code uses only one CLOUDY model to determine the emissivity as a function of $\mathbf{r}$. Later, we will also add the ability to first define a geometry with initial density parameters and calculate the needed Cloudy simulations from the specified geometry when predicting observations. This will make it possible to accurately model the emission from complex, irregular nebulae with different ionization parameters.

\section{APPENDIX B}

\section{NEW LMC AND SMC H II REGION CATALOGS}

Tables 5 and 6 present our new H II region catalogs, with the nebular boundaries defined by IPM as described in Section 3.1. Some objects are defined within the boundaries of larger background objects, and their flux is not included in the luminosity for the larger objects. The H II regions are classified by optical depth as described in Section 4, into types 0-4, corresponding to 0 -indeterminate, 1 -optically thick, 2-blister, 3-optically thin, and 4-shocked. The object ID's are listed in Columns 1 and 2, with our new designations in the former and identifications from existing catalogs in the latter. We designate new, independent substructures within a previously cataloged object by appending numbers, for example, DEM L173-1 and DEM L173-2. The object coordinates are listed in Columns 3 and 4; optical depth classifications are given in Column 5. Columns 6 
and 7 respectively give the average $N(\mathrm{HI})$ measured in the line of sight within the nebular aperture and the $\mathrm{H} \alpha$ luminosity $L$.

In Figures 23 and 24, we present representative images in $\mathrm{H} \alpha$ and $[\mathrm{S} \mathrm{II}] /[\mathrm{O} \mathrm{III}]$ of five objects in each galaxy. Each image is centered on the coordinates listed in Tables 5 and 6 . The images show the aperture used to measure the $\mathrm{H} \alpha$ flux, as well as a scale bar in both arcseconds and parsecs. When shown with dashed lines, apertures have been enlarged to reveal underlying structure. There are two labels in each image: our MCELS catalog ID in the upper left, and, centered above the object, an alternate catalog ID, unless none exists. The complete set of images can be found in the online version of this article.

\section{REFERENCES}

Abbott, D. C. 1982, ApJ, 263, 723

Arthur, S. J., Henney, W. J., Mellema, G., de Colle, F., \& Vázquez-Semadeni, E. 2011, MNRAS, 414, 1747

Azimlu, M., Marciniak, R., \& Barmby, P. 2011, AJ, 142, 139

Beckman, J. E., Rozas, M., Zurita, A., Watson, R. A., \& Knapen, J. H. 2000, AJ, 119,2728

Bica, E. L. D., Schmitt, H. R., Dutra, C. M., \& Oliveira, H. L. 1999, AJ, 117, 238

Cantalupo, S. 2010, MNRAS, 403, L16

Castellanos, M., Díaz, Á. I., \& Tenorio-Tagle, G. 2002, ApJ, 565, L79

Cowie, L. L., \& Hu, E. M. 1998, AJ, 115, 1319

Davies, R. D., Elliott, K. H., \& Meaburn, J. 1976, MmNRAS, 81, 89

Dressler, A., Martin, C. L., Henry, A., Sawicki, M., \& McCarthy, P. 2011, ApJ, 740,71

Fan, X., Narayanan, V. K., Strauss, M. A., et al. 2002, AJ, 123, 1247

Ferland, G. J., Korista, K. T., Verner, D. A., et al. 1998, PASP, 110, 761

Giammanco, C., Beckman, J. E., Zurita, A., \& Relaño, M. 2004, A\&A, 424, 877

Gnedin, N. Y., Kravtsov, A. V., \& Chen, H.-W. 2008, ApJ, 672, 765

Haffner, L. M., Dettmar, R.-J., Beckman, J. E., et al. 2009, Rev. Mod. Phys., 81, 969

Henize, K. G. 1956, ApJS, 2, 315

Heydari-Malayeri, M. 1981, A\&A, 102, 316

Hilditch, R. W., Howarth, I. D., \& Harries, T. J. 2005, MNRAS, 357, 304

Hillenbrand, L. A., \& Hartmann, L. W. 1998, ApJ, 492, 540

Hoopes, C. G., \& Walterbos, R. A. M. 2000, ApJ, 541, 597

Iglesias-Páramo, J., Boselli, A., Gavazzi, G., \& Zaccardo, A. 2004, A\&A, 421, 887

Iglesias-Páramo, J., \& Muñoz-Tuñón, C. 2002, MNRAS, 336, 33

Kehrig, C., Oey, M. S., Crowther, P. A., et al. 2011, A\&A, 526, A128

Kennicutt, R. C., Jr., Bresolin, F., Bomans, D. J., Bothun, G. D., \& Thompson, I. B. 1995, AJ, 109, 594

Kennicutt, R. C., Jr., Edgar, B. K., \& Hodge, P. W. 1989, ApJ, 337, 761

Kim, S., Staveley-Smith, L., Dopita, M. A., et al. 1998, ApJ, 503, 674

Kim, S., Staveley-Smith, L., Dopita, M. A., et al. 2003, ApJS, 148, 473

Koeppen, J. 1979, A\&AS, 35, 111

Komatsu, E., Smith, K. M., Dunkley, J., et al. 2011, ApJS, 192, 18

Lee, J. H., Hwang, N., \& Lee, M. G. 2011, ApJ, 735, 75

Macri, L. M., Stanek, K. Z., Bersier, D., Greenhill, L. J., \& Reid, M. J. 2006, ApJ, 652,1133
Martins, F., Schaerer, D., \& Hillier, D. J. 2005, A\&A, 436, 1049

Meaburn, J. 1980, MNRAS, 192, 365

Miller, W. W., III, \& Cox, D. P. 1993, ApJ, 417, 579

Oey, M. S. 2007, in IAU Symp. 237, Triggered Star Formation in a Turbulent ISM, ed. B. G. Elmegreen \& J. Palous (Cambridge: Cambridge Univ. Press), 106

Oey, M. S., \& Clarke, C. J. 1997, MNRAS, 289, 570

Oey, M. S., \& Clarke, C. J. 1998, AJ, 115, 1543

Oey, M. S., \& Kennicutt, R. C., Jr. 1997, MNRAS, 291, 827

Oey, M. S., King, N. L., \& Parker, J. W. 2004, AJ, 127, 1632

Oey, M. S., \& Lamb, J. B. 2011, arXiv:1109.0759

Oey, M. S., \& Shields, J. C. 2000, ApJ, 539, 687

Osterbrock, D. E., \& Ferland, G. J. 2006, Astrophysics of Gaseous Nebulae and Active Galactic Nuclei (2nd ed.; Sausalito, CA: Univ. Science Books)

Ostriker, E. C., McKee, C. F., \& Leroy, A. K. 2010, ApJ, 721, 975

Paardekooper, J.-P., Pelupessy, F. I., Altay, G., \& Kruip, C. J. H. 2011, A\&A, 530, 87

Paladini, R., De Zotti, G., Noriega-Crespo, A., \& Carey, S. J. 2009, ApJ, 702, 1036

Parravano, A. 1988, A\&A, 205, 71

Pellegrini, E. W., Baldwin, J. A., Brogan, C. L., et al. 2007, ApJ, 658, 1119

Pellegrini, E. W., Baldwin, J. A., \& Ferland, G. J. 2010, ApJS, 191, 160

Pellegrini, E. W., Baldwin, J. A., \& Ferland, G. J. 2011, ApJ, 738, 34

Pellegrini, E. W., Baldwin, J. A., Ferland, G. J., Shaw, G., \& Heathcote, S. 2009, ApJ, 693, 285

Pogge, R. W. 1988a, ApJ, 328, 519

Pogge, R. W. 1988b, ApJ, 332, 702

Points, S. D., Smith, R. C., \& Chu, Y.-H. 2005, BAAS, 37, 1381

Relaño, M., Peimbert, M., \& Beckman, J. 2002, ApJ, 564, 704

Reynolds, R. J. 1984, ApJ, 282, 191

Seon, K.-I. 2009, ApJ, 703, 1159

Smith, L. J., Norris, R. P. F., \& Crowther, P. A. 2002, MNRAS, 337, 1309

Smith, R. C., \& MCELS Team 1998, PASA, 15, 163

Smith, R. C., Points, S. D., Chu, Y.-H., et al. 2005, BAAS, 37, 1200

Sokasian, A., Abel, T., \& Hernquist, L. 2003, MNRAS, 340, 473

Stanimirović, S., Staveley-Smith, L., Dickey, J. M., Sault, R. J., \& Snowden, S. L. 1999, MNRAS, 302, 417

St-Louis, N., Moffat, A. F. J., Marchenko, S., \& Pittard, J. M. 2005, ApJ, 628, 953

Sullivan, M., Treyer, M. A., Ellis, R. S., \& Mobasher, B. 2004, MNRAS, 350, 21

Thilker, D. A., Walterbos, R. A. M., Braun, R., \& Hoopes, C. G. 2002, AJ, 124, 3118

Tsamis, Y. G., Barlow, M. J., Liu, X.-W., Danziger, I. J., \& Storey, P. J. 2003, MNRAS, 338, 687

Voges, E. S., Oey, M. S., Walterbos, R. A. M., \& Wilkinson, T. M. 2008, AJ, 135,1291

Walborn, N. R., Howarth, I. D., Lennon, D. J., et al. 2002, AJ, 123, 2754

Weingartner, J. C., \& Draine, B. T. 2001, ApJ, 548, 296

Winkler, P. F., Young, A. L., Braziunas, D., et al. 2005, BAAS, 37, 1380

Ye, T., Turtle, A. J., \& Kennicutt, R. C., Jr. 1991, MNRAS, 249, 722

Ye, T. S., Amy, S. W., Wang, Q. D., Ball, L., \& Dickel, J. 1995, MNRAS, 275, 1218

Zastrow, J., Oey, M. S., \& Pellegrini, E. W. 2011a, Bull. Soc. R. Sci. Liege, 80, 450

Zastrow, J., Oey, M. S., Veilleux, S., McDonald, M., \& Martin, C. L. 2011b, ApJ, 741, L17

Zurita, A., Beckman, J. E., Rozas, M., \& Ryder, S. 2002, A\&A, 386, 801 